\title{
Revision of the Anaulacaspis species of the Palaearctic region (Coleoptera: Staphylinidae: Aleocharinae)
}

\author{
With 236 figures, 7 maps and 1 key
}

VOLKER ASSING ${ }^{1}$

${ }^{1}$ Gabelsbergerstraße 2, 30163 Hannover, Germany. - vassing.hann@t-online.de Published on 2016-12-20

\section{Summary}

Types and additional material of the falagriine genus Anaulacaspis GANGLBAuER, 1895 are revised. Disregarding one name of doubtful identity, 31 species are recognized in the Palaearctic region, twelve of them new: Anaulacaspis convexa spec. nov. (Turkey); A. cristata spec. nov. (North Pakistan); A. eminens spec. nov. (South Iran, Afghanistan); A. excisa spec. nov. (South Iran); A. flavomarginata spec. nov. (Albania, Greece); A. gilva spec. nov. (North Pakistan); A. iberica spec. nov. (Spain); A. pectinata spec. nov. (China: Yunnan); A. pseudonigra spec. nov. (Turkey); A. reticulata spec. nov. (Turkey); A. sinuata spec. nov. (Iran); A. truncata spec. nov. (Iran). One species, A. inexpectata (FAGEL, 1969), is of doubtful generic assignment. The genus and the individual species are (re-)described and illustrated. Twelve synonymies are proposed: Anaulacaspis formosa (RosenHAUER, 1856) = A. cirrosa (FAUVEL, 1902), syn. n., = A. algirica (FAGEL, 1969), syn. n., = A. elkantarensis (FAGEL, 1969), syn. n., = A. macra (FAGEL, 1969), syn. n., = A. biskrensis (FAGEL, 1969), syn. n., = A. andalusiaca (FAgel, 1969), syn. n.; A. gratilla (Erichson, 1839) = A. lutzi (Reitter, 1909), syn. n.; A. naevula (Erichson, 1839) = A. elegans (CAmeron, 1944), syn. n., = A. elegansides Newton, 2015, syn. n.; A. nigra $($ Gravenhorst, 1802) = A. jonica (Bernhauer, 1910), syn. n., = A. anatolica (FAgel, 1969), syn. n., = A. corcyrana (FAGEL, 1969), syn. n. Lectotypes are designated for Aleochara nigra Gravenhorst, 1802, A. picea GravenHorst, 1802, Falagriola lutzi Reitter, 1909, Falagria laeviuscula Eppelsheim, 1880, F. nigerrima Bernhauer, 1908, and F. beesoni CAMERon, 1939. Based on morphological characters, the species are assigned to six species groups. Available data on the zoogeography and natural history of the Anaulacaspis species recorded from the Palaearctic region are compiled. The distributions of the genus as a whole and of the individual species are mapped. A catalogue and a key to species are provided.

\section{Key words}

Coleoptera, Staphylinidae, Aleocharinae, Falagriini, Anaulacaspis, Palaearctic region, taxonomy, new species, new synonymies, lectotype designations, key to species, catalogue, distribution maps

\section{Zusammenfassung}

Typen und weiteres Material der Gattung Anaulacaspis Ganglbauer, 1895, Tribus Falagriini, werden revidiert. Abgesehen von einem ungeklärten Namen werden insgesamt 31 species aus der Paläarktis nachgewiesen, davon zwölf neu: Anaulacaspis convexa spec. nov. (Türkei); A. cristata spec. nov. (Nord-Pakistan); A. eminens spec. nov. (SüdIran, Afghanistan); A. excisa spec. nov. (Süd-Iran); A. flavomarginata spec. nov. (Albanien, Griechenland); A. gilva spec. nov. (Nord-Pakistan); A. iberica spec. nov. (Spanien); A. pectinata spec. nov. (China: Yunnan); A. pseudonigra spec. nov. (Türkei); A. reticulata spec. nov. (Türkei); A. sinuata spec. nov. (Iran); A. truncata spec. nov. (Iran). Die Gattungszugehörigkeit einer Art, A. inexpectata (FAGEL, 1969), ist zweifelhaft. Die Gattung sowie die einzelnen Arten 
werden beschrieben und abgebildet. Zwölf Namen werden synonymisiert: Anaulacaspis formosa (RosENHAUER, 1856) = A. cirrosa (FAuvel, 1902), syn. n., = A. algirica (FAgel, 1969), syn. n., = A. elkantarensis (FAgEL, 1969), syn. n., = A. macra (FAgEL, 1969), syn. n., = A. biskrensis (FAgEL, 1969), syn. n., = A. andalusiaca (FAgEL, 1969), syn. n.; A. gratilla $($ Erichson, 1839) = A. lutzi (Reitter, 1909), syn. n.; A. naevula $($ Erichson, 1839) $=$ A. elegans (CAmeron, 1944), syn. n., = A. elegansides Newton, 2015, syn. n.; A. nigra (Gravenhorst, 1802)= A. jonica (Bernhauer, 1910), syn. n., = A. anatolica (FAgel, 1969), syn. n., = A. corcyrana (FAgel, 1969), syn. n. Für Aleochara nigra GravenHorst, 1802, A. picea Gravenhorst, 1802, Falagriola lutzi Reitter, 1909, Falagria laeviuscula Eppelsheim, 1880, F. nigerrima Bernhauer, 1908 und F. beesoni CAmeron, 1939 werden Lectotypen designiert. Auf der Grundlage von morphologischen Merkmalen werden die Arten sechs Artengruppen zugeordnet. Die verfügbaren Informationen zu Zoogeographie und Ökologie der aus der Paläarktis bekannten Arten werden zusammengestellt. Die Verbreitung der Gattung in der Paläarktis sowie die Verbreitungsgebiete der einzelnen Arten werden anhand von Karten illustriert. Ein Katalog und eine Bestimmungstabelle werden erstellt.

\section{Introduction}

Anaulacaspis GANGLBAUER, 1895 was originally described as a subgenus of Falagria LEACH, 1819 in a key to the subgenera of Falagria, a genus then including also Cordalia JACOBS, 1925 and the nominal subgenus. GANGLBAUER (1895) distinguished Anaulacaspis from Cordalia by the morphology of the pronotum (not cordiform and finely bordered at the margins) and from Falagria sensu strictu by the shape of the scutellum (without a deep median sulcus). He originally included four species, one of which (Falagria longipes Wollaston, 1871, a junior synonym of F. concinna ERICHSON, 1839) is in Myrmecocephalus MACLEAY, 1873 today and one (Falagria thoracica (Stephens, 1832)) in Falagrioma CAsey, 1906. One of the two remaining species, Falagria nigra (GravenHORST, 1802) was designated as the type species of Anaulacaspis by Fenyes (1912), who designated the same species also as the type species of the genus group names Melagria CAsey, 1906 and Falagriola Reitter, 1909, thus rendering these names objective junior synonyms of Anaulacaspis. Fenyes (1912) regarded Anaulacaspis as a distinct genus and included Falagrioma and Leptagria CASEY, 1906 as additional synonyms. They were, however, removed from synonymy with Anaulacaspis by HOEBEKE (1985). Although the objective synonymy of Melagria with Anaulacaspis was established as early as 1912, it was largely and until recently ignored by nearly all authors (e.g., Lohse 1974, Cameron 1939, Fagel 1969, PaCe 1998).

In a revision of the West Palaearctic species of Anaulacaspis (as Melagria), FAGEL (1969) recognized and keyed 21 species and four subspecies, and assigned them to seven species groups; eight of the species and all the subspecies were newly described.

According to Schülke \& Smetana (2015), Anaulacaspis is represented in the Palaearctic region by 30 species and four subspecies. Seven of these species are confined to the East Palaearctic region from Pakistan to China and Taiwan, one (A. nigra) has a trans-Palaearctic distribution, and the remainder has been recorded from the West Palaearctic including Middle Asia. The genus is unknown from the Nearctic region (Hobbeke 1985). Several Anaulacaspis species have been described or reported (partly as Melagria) from the Oriental, Afrotropical, and Neotropical regions, e.g., from India (CAMERon 1939), Myanmar (PACE 1986), Thailand (PACE 2004), the Malay Peninsula (CAmeron 1950), Sumatra (CAMERon 1925, 1936, PACE 2010), Borneo (CAmeron 1933), Continental Africa (Tottenham 1957), Madagascar (Pace 1999), and even Venezuela (PACE 2011). However, their generic affiliations have not been revised and the possibility that at least some of them will eventually have to be moved to other falagriine genera appears likely. This has been the case with various taxa described from the Palaearctic region. Falagria (Anaulacaspis) sherpa PACE, 1986, described from Nepal (PACE 1986b), F. (A.) rugosipennis FAGEL, 1966, described from Lebanon (FAGEL 1966), and F. (A.) yosii SAwada, 1966 from Afghanistan (SAwada 1966) were subsequently transferred to Falagrioma, and Falagria (Anaulacaspis) assamensis PACE, 1987 from Assam (PACE 1987) was moved to Leptagria (SCHÜLKe \& Smetana 2015).

As can be inferred from the remarkable number of erroneous original generic assignments and subsequent transfers to other genera, the systematic and taxonomic concept of Anaulacaspis has been largely unclear. Moreover, the most recent revision (FAGEL 1969) has rendered a reliable identification of the species recorded from the West Palaearctic practically impossible, mainly owing to inadequate illustrations of the genitalia and implausible zoogeographic concepts.

\section{Material and methods}

The material treated in this study is deposited in the following collections:

FMNH Field Museum of Natural History, Chicago (C. Maier)

HNHM Hungarian Natural History Museum, Budapest (Gy. Makranczy)

IRSNB Institut Royal des Sciences Naturelles de Belgique, Bruxelles (Y. Gérard) 
MHNG Muséum d'Histoire Naturelle, Genève (G. Cuccodoro)

MNB Museum für Naturkunde, Berlin (J. Frisch, M. Schülke, J. Willers)

MNHNP Muséum National d'Histoire Naturelle, Paris (A. Taghavian)

NHMW Naturhistorisches Museum Wien (H. Schillhammer)

NSMT National Science Museum, Tokyo (S. Nomura)

ZFMK Zoologisches Forschungsmuseum Alexander Koenig, Bonn (D. Ahrens)

cAss author's private collection

cFel private collection Benedikt Feldmann, Münster

cPüt private collection Andreas Pütz, Eisenhüttenstadt

cTro private collection Marc Tronquet, Molitg-lesBains

cWun private collection Paul Wunderle, Mönchengladbach

cZan private collection Adriano Zanetti, Verona

The morphological studies were conducted using a Stemi SV 11 microscope (Zeiss Germany) and a Jenalab compound microscope (Carl Zeiss Jena). The images were taken using a digital camera (Nikon Coolpix 995). The maps were created using MapCreator 2.0 (primap) software.

Body length was measured from the anterior margin of the labrum to the apex of the abdomen, the length of the forebody from the anterior margin of the labrum to the posterior margin of the elytra, head length from the anterior margin of the clypeus (without ante-clypeus) to the posterior constriction of the head, the length of the elytra along the suture from the apex of the scutellum to the posterior margin of the elytra, and the length of the median lobe of the aedeagus from the apex of the ventral process to the base of the aedeagal capsule. The "parameral" side of the median lobe of the aedeagus (i.e., the side where the sperm duct enters) is referred to as the ventral, the opposite side as the dorsal aspect.

\section{Results}

\subsection{The genus Anaulacaspis}

Redescription: Body of rather small size; body length $1.6-3.3 \mathrm{~mm}$; length of forebody $0.8-1.5 \mathrm{~mm}$. Coloration variable. Habitus as illustrated in Fig. 211.

Head more or less distinctly transverse, with or without sexual dimorphism, in male often depressed or shallowly impressed in median or postero-median portion of dorsal surface and/or with more distinct punctation than in female; posterior constriction approximately onethird as broad as maximal head width; ventral aspect of head smooth; gular sutures broadly separated. Eyes rather large, slightly shorter, approximately as long as, or longer than postocular region in dorsal view. Antenna of moderate length, weakly to distinctly incrassate apically. Labrum strongly transverse, shape and chaetotaxy as in Fig. 3. Right mandible with very obtuse, sometimes indistinct molar tooth in the middle; left mandible without molar tooth. Maxilla and labium as in Figs 1-2.

Pronotum more or less distinctly transverse, broadest at or near anterior angles, tapering posteriad; posterior angles obtuse, moderately marked; along midline rarely with, usually without weakly pronounced median sulcus of reduced length posteriorly, this sulcus (if present) anteriorly not, or only slightly, extending beyond middle of pronotal length (never as deep and long as in Falagria); with or without sexual dimorphism: in male often with median depression, impression, or sulcus in posterior half, and/or with more distinct punctation than in female. Prosternite strongly transverse, in the middle elevated, but not carinate.

Elytra of variable length and width; scutellum without sulcus, flat, and usually densely punctate, in one species (A. inexpectata) with coarse, irregularly longitudinal rugae. Process of mesoventrite broad and apically obtusely truncate, reaching approximately halfway between mesocoxal cavities; mesocoxal cavities not margined posteriorly; metaventrite long, much longer than mesoventrite. Metatarsomere I elongate, at least approximately as long as the combined length of metatarsomeres II-IV.

Abdomen broadest at tergite $\mathrm{V}$; tergites III-V with more or less distinct anterior impressions, impression of tergite $\mathrm{V}$ as deep as those of tergites III-IV, or shallower; anterior impressions of tergites III-V smooth and impunctate, finely punctate, or coarsely and densely punctate; sternites III-V constricted anteriorly; punctation of posterior tergites finer and sparser than that of anterior tergites, often also finer and sparser on posterior portions than on anterior portions of tergites; tergite VII with palisade fringe; tergite VIII anteriorly with a more or less pronounced median excision (e.g., Figs 4-9) (exception: A. inexpectata), with or without weakly pronounced sexual dimorphism; posterior margin of tergite VIII with thin (unmodified) or with more or less distinctly modified marginal setae; sternite VIII with more or less pronounced sexual dimorphism.

$\mathrm{o}^{\top}$ : posterior margin of sternite VIII more or less distinctly produced in the middle; median lobe of aedeagus of variable size (in relation to body size) and usually of simple structure; paramere distinctly divided into paramerite and condylite; condylite without distinct velum, much narrower and shorter than the paramerite; apical lobe of paramerite short.

\%: posterior margin of sternite VIII broadly convex, often weakly concave in the middle; spermatheca minute, short, and comma-shaped (Figs 186, 210) (exception: A. inexpectata).

Comparative notes: Except for A. inexpectata, Anaulacaspis is distinguished from all other genera of Falagriini 
recorded from the Palaearctic region by the median excision of the anterior margin of tergite VIII and by the minute comma-shaped spermatheca. In addition, it differs from similar falagriine genera as follows:

from Falagria by the absence of a long and deep median sulcus on the pronotum and on the scutellum, as well as by the chaetotaxy of tergite VIII (Falagria: whole posterior margin with a very dense fringe of short modified setae);

from Falagrioma by the absence of a long and deep median sulcus on the pronotum, by the different punctation of the elytra (Falagrioma: punctation conspicuously dense in antero-median portion), and by the chaetotaxy of tergite VIII (Falagrioma: posterior margin with a dense fringe of moderately short modified setae);

from Cordalia by a less globulous pronotum and by the chaetotaxy of tergite VIII (Cordalia: tergite VIII conspicuously modified, at least posterior portion with dense fringes of fur-like long pubescence).

All other falagriine genera known from the Palaearctic region are readily distinguished from Anaulacaspis by different habitus and/or size alone.

Species groups: The speciose $A$. nigra group includes A. nigra, A. caucasica, A.pseudonigra, A. reticulata, A. libanotica, A. sinuata, A.nigerrima, A.persica, A. truncata, A. convexa, A. eminens, and A. gratilla. It is characterized by small body size, mostly dark coloration (exception: A. persica and allied species, A. gratilla), in most species a more or less distinct, but generally weakly pronounced sexual dimorphism of the head and/or the pronotum, a deep and rather narrow median excision of the anterior margin of tergite VIII, the derived chaetotaxy of tergite VIII (postero-laterally with a more or less pronounced cluster of dense long thin setae on either side; posterior margin with unmodified marginal setae), and a small median lobe of the aedeagus (in relation to body size). At least the postero-lateral clusters of setae of tergite VIII and the deep median excision of the anterior margin of tergite VIII most likely represent synapomorphies constituting the monophyly of this group. Within the $A$. nigra group, the species allied to $A$. persica (A. persica, A.truncata, A. convexa, A. eminens) form a distinct monophylum constituted by the modified tergite VIII (posterior margin strongly convex or conspicuously projecting the middle).

Thirteen species are assigned to the A. naevula group, some of them only tentatively: A. naevula, A. seclusa, A. desertorum, A.cristata, A. excisa, A.pamphylica, A. flavomarginata, A. nigrina, A. laevigata, A. iberica, A. beijingensis, A. taiwanica, and A. schuelkei. They share the absence of distinct postero-lateral clusters on tergite VIII, unmodified marginal setae at the posterior margin of tergite VIII (exceptions: A. pectinata, A. gilva), and an often large median lobe of the aedeagus (in relation to body size). Moreover, the median excision of the anterior margin of tergite VIII is shallower and broader than in the $A$. nigra group, the posterior margin of the male sternite VIII is more or less obtusely pointed in the middle (not distinctly produced), and the posterior margin of the female sternite VIII is usually weakly concave in the middle. Anaulacaspis taiwanica (shape and chaetotaxy of tergite VIII sexually dimorphic) and A. schuelkei (male sternite VIII distinctly produced posteriorly) are only tentatively assigned to this group. There is little doubt that $A$. laevigata and A. iberica represent sister species, as can be inferred from their highly similar external and secondary sexual characters. They form a distinct lineage together with A. nigrina, with which they share an extremely fine and sparse punctation of the forebody and the absence of a sexual dimorphism of the head and pronotum.

The two species of the A. gilva group, A. gilva and A. pectinata, in some respects resemble those of the A. naevula group, but are distinguished by the absence of a sexual dimorphism of the head and pronotum, extremely fine and rather sparse punctation of the forebody, by a distinct comb of modified setae at the posterior margin of tergite VIII, and by a conspicuously derived morphology of the aedeagus (ventral process very long and slender both in lateral and in ventral view; internal sac with a long dark structure).

The monophyly of the A. formosa group, which contains only A. formosa and A. elegans, is constituted by the synapomorphically modified chaetotaxy of tergite VIII (posterior margin with distinctly modified short and stout setae, Figs 197, 219). In other respects both species are similar to the representatives of the A. naevula group. Whether this lineage is nested within the A. naevula group or represents a distinct lineage is uncertain. Until this question is clarified, the $A$. formosa group is regarded as a separate lineage.

The monotypical A. beesoni group is distinguished from all other Palaearctic representatives of the genus by the conspicuously derived chaetotaxy of tergite and sternite VIII (postero-laterally with clusters of modified, apically dilated setae, Fig. 223).

The generic assignment of A. inexpectata is doubtful. Though similar in habitus, punctation, the shape of the pronotum, the morphology of the scutellum, and numerous other characters, it lacks two presumable synapomorphies of other Anaulacaspsis species (median excision of the anterior margin of tergite VIII; minute and comma-shaped spermatheca) and additionally differs from all other Anaulacaspis species by a conspicuously rugose scutellum. However, since the possibility that these characters represent derived characters (autapomorphies) cannot be ruled out and since there are no evident synapomorphies suggesting that it should belong to any other described genus of Falagriini, the species is tentatively retained in Anaulacaspis.

Intraspecific variation and polymorphism: Many species are subject to remarkable intraspecific variation of coloration and also size. A pronounced polymorphism of the shape and length of the elytra (and probably also of the 
length of the hind wings) was observed for Anaulacaspis nigra and $A$. formosa. In the former, specimens from populations in West and Central Europe usually have short and relatively narrow elytra, whereas those from Southeast Europe and Asia tend to have distinctly longer and broader elytra. Similarly, material of $A$. formosa from Spain is generally characterized by short and narrow elytra, while those of specimens from North Africa tend to be distinctly longer and broader. However, intermediate forms were observed in Morocco and Algeria, and Moroccan populations may be composed of both longand short-winged morphs.

Remarkable intraspecific variation was not only observed for external, but also for the male primary and secondary sexual characters. This is particularly true of the aedeagus of A. nigra, which may considerably vary in size. Moreover, the semi-transparent median carina at the base of the ventral process may be pronounced (most populations) to almost missing (populations in Corfu, the Pelopónnisos, and in Albania). Finally, while the male tergite VIII is weakly concave in most populations, it may be strongly concave in material from Bulgaria.

Identification: Disregarding A. nigra, which is the sole representative of the genus in much of Europe, more than half of the examined specimens were either misidentified, or they had been identified based on a misinterpretation of the respective species. Consequently, previous literature records are mostly unreliable, particularly those from regions where several species are present.

In view of the pronounced intraspecific variation of characters such as the coloration, size, modifications of the male head and pronotum, and the length of the elytra on the one hand, and often low interspecific variation not only of external characters but also of the spermatheca on the other, the best character for a reliable identification is the shape of the median lobe of the aedeagus. The shape and chaetotaxy of tergite VIII often provide additional diagnostic characters. Nevertheless, a positive identification of females may not always be possible. The only regions where Anaulacaspis species can be identified with sufficient reliability based on external characters alone are Europe exclusive of the southeast and North Africa.

Diversity, zoogeography, and natural history: The overall distribution of Anaulacaspis is currently unknown (see introduction). The genus is currently represented in the Palaearctic region sensu SCHÜLKE \& SMETANA (2015) by 31 species (disregarding a doubtful name), twelve of which are described for the first time. The generic assignment of one species is only tentative (see section on species groups above).

Except for the widespread A. nigra, Anaulacaspis species are confined to warmer climates in the south of the Palaearctic region (Map 1). Some of them inhabit temporarily or permanently moist habitats in generally arid and semi-arid regions. Diversity hotspots in the Palaearctic region are the East Mediterranean (particularly Turkey), the Middle East (especially Iran), the Caucasus region, and Middle Asia. The countries with the greatest diversity are Iran (twelve species, three of them exclusive) and Turkey (six species, four exclusive), followed by Greece, Afghanistan, and Kyrgyzstan (four species each).

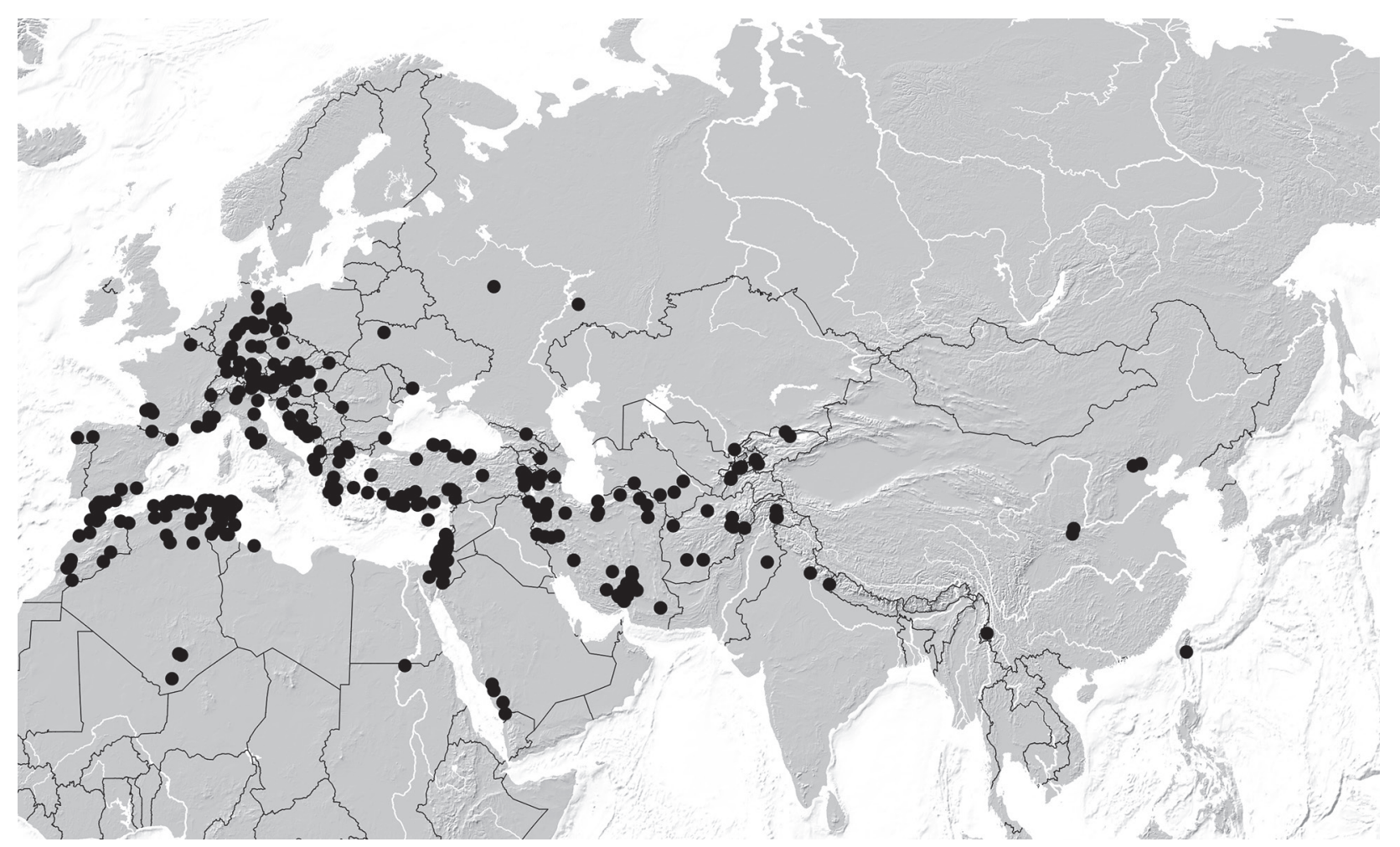

Map 1: Distribution of Anaulacaspis in the Palaearctic region, based on revised records (all records pooled). 
Some of the species are remarkably widespread. This particularly applies to Anaulacaspis nigra, whose confirmed distribution extends from West Europe to southern Turkey and Middle Asia. Several species, by contrast, are currently known only from their respective type localities.

Species of Anaulacaspis are generally found in moist habitats with sparse vegetation, mostly on the banks of temporary and permanent streams and rivers or on the shores of standing waters. At least one species, A. laevigata, may inhabit also swampy habitats. Anaulacaspis nigra has been found in a wide range of habitats such as different types of grassland, near running and standing waters, and even in nests of ants and mice.

\subsection{Catalogue}

The doubtful name A. medvedevi (Kashcheev, 1982) is omitted from the catalogue below.

\begin{tabular}{|c|c|}
\hline Species & Distribution \\
\hline beesoni (CAMERoN, 1939) & North India (Uttarakhand, Uttar Pradesh) \\
\hline $\begin{array}{l}\text { beijingensis (PACE, 1998) } \\
=\text { marginata (PACE, 1998); syn. } \mathbf{n} .\end{array}$ & China (Beijing, Shaanxi) \\
\hline caucasica (FAGEL, 1969) & Azerbaijan; North Iran \\
\hline convexa sp. $\mathrm{n}$. & Turkey \\
\hline cristata sp. n. & North Pakistan \\
\hline desertorum (FAUVEL, 1898) & $\begin{array}{l}\text { North Africa and Middle East: Morocco; Algeria; Tunisia; Jordan; Saudi } \\
\text { Arabia }\end{array}$ \\
\hline elegans (BAUDI DI SELVE, 1857) & Middle East: Cyprus; Lebanon; Israel; Jordan; Saudi Arabia; Egypt (Sinai) \\
\hline eminens sp. $\mathbf{n}$. & South Iran; Afghanistan \\
\hline excisa sp. n. & South Iran \\
\hline flavomarginata sp. $\mathbf{n}$. & Albania; Greece \\
\hline $\begin{array}{l}\text { formosa (RosENHAUER, 1856) } \\
=\text { cirrosa (FAUVEL, 1902); syn. n. } \\
=\text { algirica (FAGEL, 1969); syn. } \mathbf{n} . \\
=\text { elkantarensis (FAGEL, 1969); syn. n. } \\
=\text { macra (FAGEL, 1969); syn. } \mathbf{n} . \\
=\text { biskrensis (FAGEL, 1969); syn. } \mathbf{n} . \\
=\text { andalusiaca (FAGEL, 1969); syn. } \mathbf{n} .\end{array}$ & South Spain; Morocco; Algeria; Tunisia; Libya \\
\hline gilva sp. $\mathbf{n}$. & North Pakistan \\
\hline $\begin{array}{l}\text { gratilla (ERICHSON, 1839) } \\
=\text { lutzi (REITTER, 1909); syn. n. }\end{array}$ & Ukraine; Iran; Iraq; Azerbaijan; Uzbekistan; Kazakhstan; Afghanistan \\
\hline iberica sp. n. & Spain (Galicia, Andalucía) \\
\hline inexpectata (FAGEL, 1969) & Iran; Kyrgyzstan \\
\hline $\begin{array}{l}\text { laevigata (DuvIVIER, 1883) } \\
\text { = laeviuscula (EPPELSHEIM, 1880) }\end{array}$ & $\begin{array}{l}\text { Italy; Croatia; Montenegro; Bosnia-Herzegovina; Greece (Pelopónnisos, } \\
\text { Corfu, Lesbos); Turkey }\end{array}$ \\
\hline libanotica (FAGEL, 1969) & Lebanon; Israel \\
\hline $\begin{array}{l}\text { naevula (ERICHSON, 1839) } \\
=\text { nilotica }(\mathrm{KOCH}, 1936) \\
=\text { elegans (CAMERON, 1944); syn. } \mathbf{n} . \\
=\text { elegansides NEWTON, 2015; syn. } \mathbf{n} .\end{array}$ & North Sudan; Saudi Arabia; South Iran; Pakistan \\
\hline nigerrima (BERNHAUER, 1908) & $\begin{array}{l}\text { Middle Asia: Northeast Iran; Turkmenistan; Kyrgyzstan; Uzbekistan; } \\
\text { Tajikistan; Afghanistan; "Siberia or." }\end{array}$ \\
\hline
\end{tabular}




\begin{tabular}{|c|c|}
\hline Species & Distribution \\
\hline $\begin{array}{l}\text { nigra (GRAVENHORST, 1802) } \\
=\text { picea (GRAVENHORST, 1802) } \\
=\text { fracticornis (GRAVENHORST, 1806) } \\
=\text { pusilla (HEER, 1839) } \\
=\text { jonica (BERNHAUER, 1910); syn. n. } \\
=\text { anatolica (FAGEL, 1969); syn. } \mathbf{n} . \\
=\text { corcyrana (FAGEL, 1969); syn. } \mathbf{n} .\end{array}$ & $\begin{array}{l}\text { Europe (except for the southwest and the British Isles); Turkey; Armenia; } \\
\text { Georgia; Kyrgyzstan }\end{array}$ \\
\hline nigrina (FAGEL, 1969) & Greece (Lesbos); Turkey; Iran \\
\hline pamphylica (FAGEL, 1969) & Turkey (Antalya) \\
\hline pectinata sp. n. & China (Yunnan) \\
\hline persica (FAGEL, 1969) & Iran; Kyrgyzstan \\
\hline pseudonigra sp. n. & Turkey (Amasya) \\
\hline reticulata sp. n. & Turkey (Mersin) \\
\hline schuelkei (Assing, 2001) & China (Shaanxi) \\
\hline $\begin{array}{l}\text { seclusa (FAGEL, 1969) } \\
=\text { klapperichi (COIFFIT, 1982); syn. n. }\end{array}$ & Iran; Turkmenistan; Afghanistan \\
\hline sinuata sp. $\mathbf{n}$. & Iran (Kordestan, Lorestan) \\
\hline taiwanica PACE, 2008 & Taiwan \\
\hline truncata sp. n. & Iran (Azarbayjan-e Gharbi) \\
\hline
\end{tabular}

\subsection{Key to species}

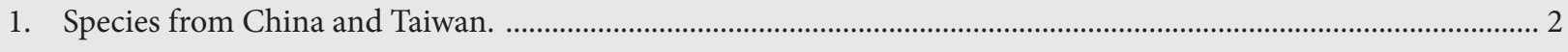

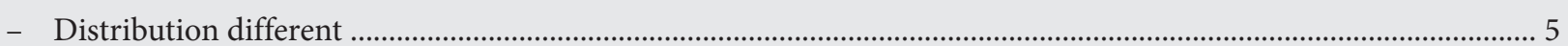

2. Pronotum and most of elytra blackish-brown to black. ........................................................................................ 3

- Pronotum and most of elytra pale-reddish to pale-brown. ...................................................................................... 4

3. Male pronotum with shallow impression. Antenna without sexual dimorphism. Tergite VIII sexually dimorphic, in male with broadly concave and in female with truncate posterior margin (Figs 178, 182). Median lobe of aedeagus shaped as in Figs 180-181. Distribution: Taiwan (Map 7). taiwanica

- Male pronotum with deep and extensive impression. Antenna sexually dimorphic, longer and more slender in male than in female. Tergite VIII (Fig. 173) strongly tranverse and with truncate posterior margin, without sexual dimorphism. Median lobe of aedeagus as in Figs 175-176. Distribution: China: Beijing, Shaanxi (Map 7).... beijingensis

4. Antennomere XI pale-brown, paler than antennomeres V-X. Male head depressed dorsally. Posterior margin of tergite VIII concave and with unmodified thin setae (Assing 2001: figure 12). Median lobe of aedeagus with broad (ventral view) and strongly curved (lateral view) ventral process (Assing 2001: figures 10-11). Distribution: China: Shaanxi (Map 7). schuelkei

- Antennomere XI blackish-brown, not paler than antennomeres V-X. Male head unmodified, not depressed dorsally. Posterior margin of tergite VIII convex and with a comb-like row of modified, long and rather stout setae (Figs 191-192). Median lobe of aedeagus slender, with very slender and apically acute ventral process and with long dark structure in internal sac (Figs 194-195). Distribution: China: Yunnan (Map 7). pectinata

5. Whole body dark-brown to black, sometimes with the posterior margin of the elytra more or less distinctly paler. Small species; length of forebody usually $<1.2 \mathrm{~mm}$, only in one species from the Middle East up to $1.3 \mathrm{~mm}$ long.

- Body partly paler; at least the humeral angles and the suture of the elytra, usually also the pronotum and parts of the abdomen, yellowish to reddish. 
6. Scutellum coarsely rugosely sculptured. Anterior impressions of tergites III-V deep and with coarse punctures separated by longitudinal carinae. Tergite VIII without anterior median excision (Fig. 233); posterior margin with a row of dense short modified setae (Fig. 234). Median lobe of aedeagus as in Figs 229-232. Spermatheca with long capsule, not comma-shaped. Distribution: Iran, Kyrgyzstan (Map 5)

inexpectata

- Scutellum neither coarsely nor rugosely sculptured. Anterior impressions with fine punctation and without carinae. Tergite VIII with anterior median excision; posterior margin with unmodified setae. Spermatheca comma-shaped.

7. Posterior margin of elytra sharply yellow.

- Elytra either of uniformly dark coloration or with diffusely paler posterior margin. 9

8. Posterior portion of male head depressed or shallowly impressed. Male pronotum with extensive and rather deep impression. Median lobe of aedeagus larger, approximately $0.38 \mathrm{~mm}$ long, and shaped as in Figs 144-145. Distribution: South Turkey (Antalya) (Map 5).

pamphylica

- Male head without appreciable depression or impression posteriorly. Male pronotum without distinct impression. Median lobe of aedeagus smaller, approximately $0.30 \mathrm{~mm}$ long, and shaped as in Figs 148-149. Distribution: Albania, Greece (Map 5)

flavomarginata

9. Pronotum strongly transverse, approximately 1.2 times as broad as long. Forebody with fine punctation. Posterolateral portions of tergite and sternite VIII each with a cluster of conspicuously modified, apically dilated setae (Fig. 223). Median lobe of aedeagus as in Figs 224-227. Distribution: North India (Map 7). beesoni

- Pronotum less transverse, usually 1.10-1.15 times as broad as long. Postero-lateral portions of sternite VIII without clusters of setae; postero-lateral portions of tergite VIII often with clusters of setae, but these setae long and thin, not dilated apically. Unknown from India.

10

10. Head and pronotum with extremely fine punctation (slightly more distinct in male A. nigrina). Tergite VIII posterolaterally with or without cluster of long thin setae on either side. Male pronotum with indistinct impression at most.

- Head with fine, but distinct punctation. Tergite VIII postero-laterally always with cluster of long thin setae on either side. Male pronotum with dense and distinct punctation and often with median impression posteriorly.

15

11. Tergite VIII postero-laterally with a cluster of long thin setae on either side, anterior margin with narrower and deeper median excision. Male sternite VIII strongly produced posteriorly. Posterior margin of female sternite VIII convex, not concave in the middle.

12

- Tergite VIII postero-laterally without a cluster of long thin setae on either side, anterior margin with broader and shallower median excision. Male sternite VIII less produced posteriorly, broadly convex to obtusely pointed. Posterior margin of female sternite VIII concave in the middle.

12. Posterior margin of tergite VIII truncate (Fig. 52). Male sternite VIII more strongly produced posteriorly (Fig. 53). Median lobe of aedeagus 0.21-0.23 mm long and shaped as in Figs 54-55. Distribution: West Iran (Map 2).

sinuata

- Posterior margin of tergite VIII concave (Fig. 57). Male sternite VIII obtusely pointed posteriorly. Median lobe of aedeagus larger, $0.27-0.30 \mathrm{~mm}$ long, and shaped as in Figs 59-61. Distribution: Middle East from Northeast Iran eastwards to Kyrgyzstan and Afghanistan (Map 3).

nigerrima

13. Male head with or without indistinct depression in postero-median portion. Male pronotum shallowly and usually extensively depressed or impressed in the middle). Posterior margin of tergite VIII shallowly concave (Fig. 167). Median lobe of aedeagus as in Figs 169-171. Distribution: Greek island Lesbos, Turkey, Iran (Map 3). .... nigrina

- Head and pronotum without sexual dimorphism. Posterior margin of tergite VIII truncate to weakly convex.... 14

14. Median lobe of aedeagus (Figs 159-163) slightly smaller; ventral process longer in relation basal capsule, apically more slender in lateral view; crista apicalis more prominent. Posterior margin of female sternite VIII more distinctly concave in the middle (Fig. 166). Distribution: Spain (Map 4).

iberica

- Median lobe of aedeagus (Figs 153-157) slightly larger; ventral process shorter in relation to basal capsule, apically less slender in lateral view; crista apicalis less prominent. Posterior margin of female sternite VIII very shallowly concave in the middle (Fig. 158). Distribution Ponto-Mediterranean: Turkey, Balkans, Italy (Map 4). ... laevigata 
15. Ventral process of aedeagus in ventral view with a pronounced projection on either side (Figs 34, 39-40). ...... 16

- Ventral process of aedeagus in ventral view sometimes angled laterally, but without pronounced projection.

16. Median lobe of aedeagus $0.24-0.26 \mathrm{~mm}$ long and shaped as in Figs 37-40. Distribution: North Turkey: Amasya (Map 2). pseudonigra

Median lobe of aedeagus larger, approximately $0.28 \mathrm{~mm}$ long, and shaped as in Figs 33-34. Distribution: Azerbaijan, North Iran (Map 2).

caucasica

17. Distribution: Middle East (Israel, Lebanon) (Map 2). Median lobe of aedeagus larger, approximately $0.32 \mathrm{~mm}$ long, and shaped as in Figs 47-48. libanotica

- Absent from the Middle East. Median lobe of aedeagus smaller. 18

18. Antennomere IV distinctly transverse. Pronotum, elytra, and abdomen with distinct microreticulation (Fig. 42). Posterior margin of tergite VIII convex (Fig. 43). Median lobe of aedeagus $0.21 \mathrm{~mm}$ long and shaped as in Figs 45-46; ventral process broad in ventral view and without membranous projection at base (lateral view). Distribution: Turkey (Mersin) (Map 2). reticulata

- Antennomere IV approximately as long as broad. Pronotum and elytra without appreciable microreticulation. Posterior margin of tergite VIII weakly to strongly concave (Figs 4-9). Median lobe of aedeagus larger, 0.23$0.28 \mathrm{~mm}$ long; ventral process of different shape, at base usually with, rarely without, membranous projection in lateral view (Figs 13-30). Widespread from northeastern Spain and France eastwards to Kyrgyzstan (Map 2).

nigra

19. Tergite VIII postero-laterally with a cluster of long thin setae on either side. Small species; length of forebody $1.2 \mathrm{~mm}$ at most. Posterior margin of tergite VIII with conspicuous median projection or strongly convex, except in A. gratilla.

- Tergite VIII postero-laterally without cluster of long thin setae on either side. Mostly larger species. Posterior margin of tergite VIII always without median process.

20. Posterior margin of tergite VIII truncate to convex, without a conspicuous median process.

- Posterior margin of tergite VIII with a conspicuous median process (Figs 62-63, 70, 74, 76, 82). Male sternite VIII more strongly produced posteriorly.

21. Posterior margin of tergite VIII truncate to weakly convex (Fig. 121). Median lobe of aedeagus shaped as in Figs 123-126, 130-131. Posterior margin of female sternite VIII weakly concave in the middle (Fig. 127). Widespread from Ukraine in the west to Middle Asia in the east (Map 4). gratilla

- Posterior margin of tergite VIII strongly convex (Figs 84, 89). Median lobe of aedeagus with strongly prominent crista apicalis and with ventral process of completely different shape (Figs 86-88). Posterior margin of female sternite VIII convex, without median concavity. Distribution: South Iran, Afghanistan (Map 3).

eminens

22. Median projection of posterior margin of tergite VIII narrower (Figs 62-63). Median lobe of aedeagus shaped as in Figs 65-69. Distribution: Iran, Kyrgyzstan (Map 5). persica

Median projection of posterior margin of tergite VIII broader. Median lobe of aedeagus of different shape. .....

23. Tergite VIII with broader and apically distinctly truncate median projection posteriorly (Figs 70, 74). Median lobe of aedeagus shaped as in Figs 72-73. Distribution: Northwest Iran (Map 2). truncata

- Tergite VIII with less broad and apically convex median projection posteriorly (Figs 76, 82). Median lobe of aedeagus as in Figs 78-81, with distinctly broader ventral process in ventral view. Distribution: Turkey (Map 6).

convexa

24. Posterior margin of tergite VIII with a row of strongly modified, stout and very short setae (Figs 197, 219). .... 25

- Posterior margin of tergite VIII mostly with unmodified, in one species with weakly modified long setae. ...... 26

25. Pronotum with weakly pronounced sexual dimorphim; male pronotum with or without depression or very shallow median impression posteriorly. Median lobe of aedeagus (Figs 199-208) with relatively shorter ventral process. Distribution: South Spain, Northwest Africa eastwards to Libya (Map 3). formosa 
Pronotum with pronounced sexual dimorphism; male pronotum with distinct and extensive median impression. Median lobe of aedeagus with relatively longer ventral process (Figs 214-217). Distribution: Middle East (Cyprus, Lebanon, Israel, Jordan, Sinai Peninsula, Saudi Arabia) (Map 3).

elegans

26. Median lobe of aedeagus with crista apicalis of very conspicuous shape (Figs 134-135). Distribution: North Pakistan (Map 5). cristata

- Median lobe of aedeagus of completely different shape. 27

27. Posterior margin of tergite VIII with a comb-like row of rather long and slightly modified setae (Figs 184, 189). Median lobe of aedeagus very slender, with long, slender, and apically acute ventral process, and with long dark structure in internal sac (Figs 187-188). Distribution: North Pakistan (Map 7). gilva

- Posterior margin of tergite VIII with unmodified setae. Median lobe of aedeagus much less slender and without long dark structure in internal sac. 28

28. Antenna very slender, $1.2-1.3 \mathrm{~mm}$ long; antennomeres IV-VII distinctly oblong, VIII-IX approximately as long as broad, and X weakly transverse. Posterior margin of tergite VIII (Figs 136, 140) concavely excised in the middle, setae at posterior margin short, middle of posterior margin without marginal setae. Median lobe of aedeagus shaped as in Figs 138-139; ventral process subapically obtusely angled in lateral view. Distribution: Iran (Map 4). excisa

- Antenna less slender. Posterior margin of tergite VIII without median concavity and with uninterrupted row of longer setae. Median lobe of aedeagus of different shape. 29

29. Coloration generally paler; head usually reddish to reddish-brown; medio-lateral infuscate spot on elytra usually brown to dark-brown; abdominal segments V-VII usually brown to dark-brown. Median lobe of aedeagus shaped as in Figs 93-100. Distribution: North Sudan, Saudi Arabia, South Iran, Pakistan (Map 4). naevula

- Coloration generally darker; head usually dark-brown to blackish; medio-lateral infuscate spot on elytra darkbrown to black, usually larger and more defined; abdominal segments V-VII blackish-brown to black. 30

30. Pronotum with weakly pronounced sexual dimorphism, in male with shallow and small impression. Median lobe of aedeagus with apically longer and more slender ventral process in lateral view (Figs 110-111, 115-118). Distribution: Iran, Turkmenistan, Afghanistan (Map 6). seclusa

- Pronotum with pronounced sexual dimorphism, in male with deep and extensive impression. Median lobe of aedeagus with apically shorter and less slender ventral process in lateral view (Figs 104-107). Distribution: North Africa and Middle East (Jordan, Saudi Arabia) (Map 4). desertorum

\subsection{The Anaulacaspis species of the Palaearctic region}

\section{Anaulacaspis nigra (GraveNHORST, 1802)} (Figs 1-30, Map 1)

Aleochara nigra GravenHORST, 1802: 75.

Aleochara picea GRAVENHORST, 1802: 75.

Aleochara fracticornis GRAVENHORST, 1806: 152.

Falagria pusilla HeER, 1839: 351.

Falagria nigra var. jonica Bernhauer, 1910: 78; syn. n.

Melagria anatolica FAGEL, 1969: 9 ff.; syn. $\mathbf{n}$.

Melagria corcyrana FAGEL, 1969: 8 f.; syn. n.

Type material examined: Aleochara nigra: Lectotype $0^{*}$, present designation: "Hist.-Coll. (Coleoptera), Nr. 5297, Falagria nigra Gyllh., Germania, Zool. Mus. Berlin / Syntypus Aleochara nigra Gravenhorst, 1802, labelled by MNB 2011 / Lectotypus ơ Aleochara nigra Gravenhorst, desig. V. Assing 2016 / Anaulacaspis nigra (Gravenhorst), det. V. Assing 2016" (MNB). Paralectotypes: $10^{\star}, 4$ 우 우: same data as lectotype (MNB).
Aleochara picea: Lectotype $\sigma^{\star}$, present designation: "Hist.-Coll. (Coleoptera), Nr. 5297, Falagria nigra Gyllh., Germania, Zool. Mus. Berlin / Syntypus Aleochara picea Gravenhorst, 1802, labelled by MNB 2011 / Lectotypus o Aleochara picea Gravenhorst, desig. V. Assing 2016 / Anaulacaspis nigra (Gravenhorst), det. V. Assing 2016" (MNB). Paralectotype + : same data as lectotype (MNB). Falagria jonica: Lectotype + : “Gr. Attica, Bang-Haas / jonica / v. jonica Brh, Cotypus / Chicago NHMus, M. Bernhauer Collection / Lectotypus Fagel 1969 / Anaulacaspis nigra (Gravenhorst), det. V. Assing 2016" (FMNH).

Melagria anatolica: Holotype ơ: "Anatolie mér., Alanya (plage), VI.1968 G. Fagel / G. Fagel det., anatolica n. sp. / Type / Anaulacaspis anatolica (Fagel), det. V. Assing 2016" (IRSNB). Paratypes: 1 : same data as holotype (IRSNB); 1 : : "Anatolie mér., Alanya: Dim Irmak, VI.1968 G. Fagel / G. Fagel det., anatolica n. sp. / Paratype / Anaulacaspis nigra (Gravenhorst), det. V. Assing 2016” (IRSNB).

Melagria corcyrana: Holotype $0^{\star}$ [dissected prior to present study; aedeagus damaged considerably]: "Corfu, Reitter. / G. Fagel det., corcyrana n. sp. / Type / Anaulacaspis corcyrana (Fagel), det. V. Assing 2016” (IRSNB). Paratype 9 : same data as holotype (IRSNB). 


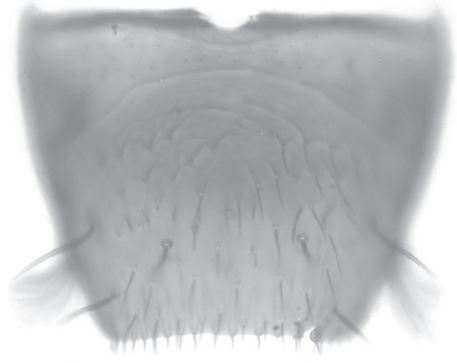

4

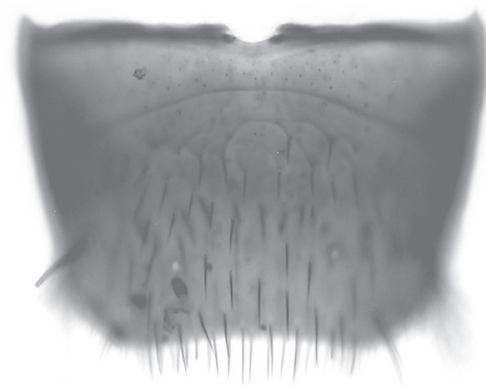

7

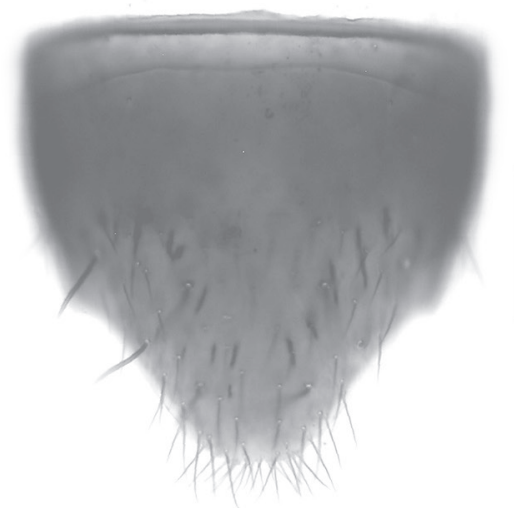

10

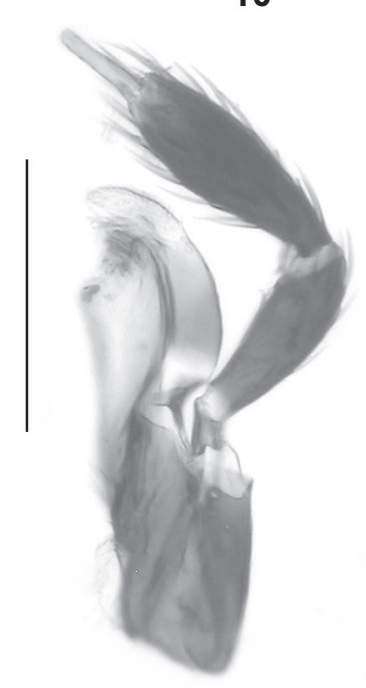

1

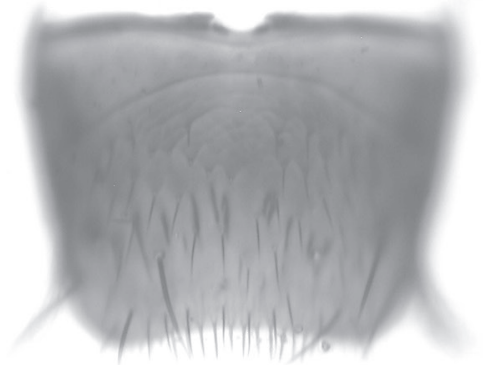

5

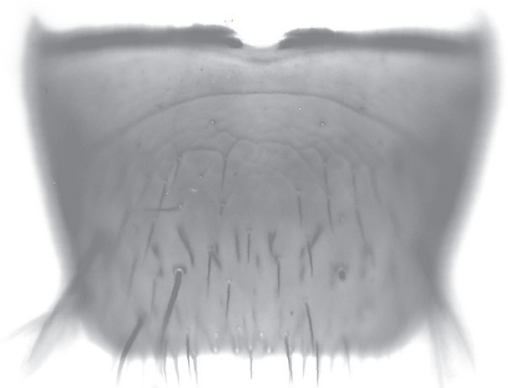

8

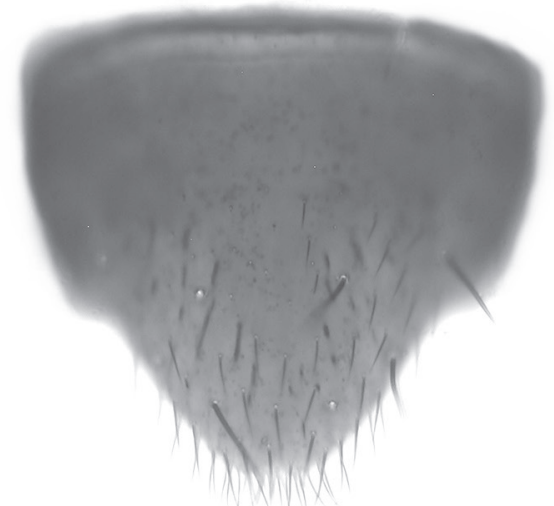

11

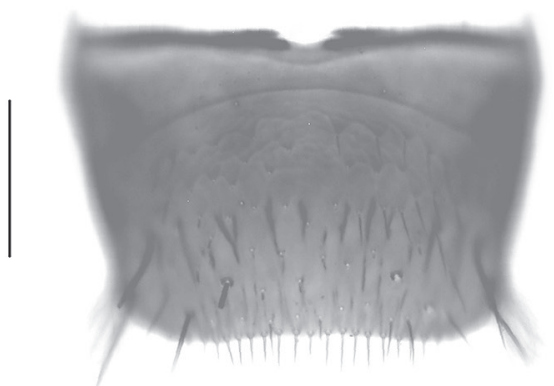

6

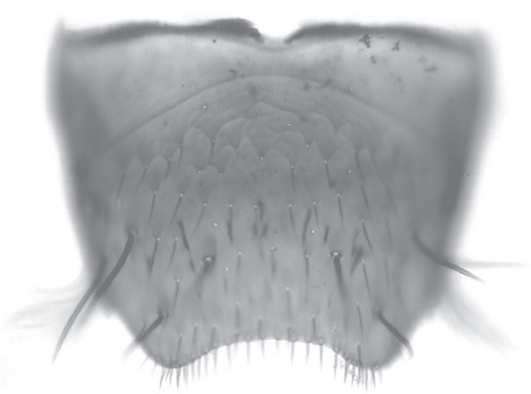

9

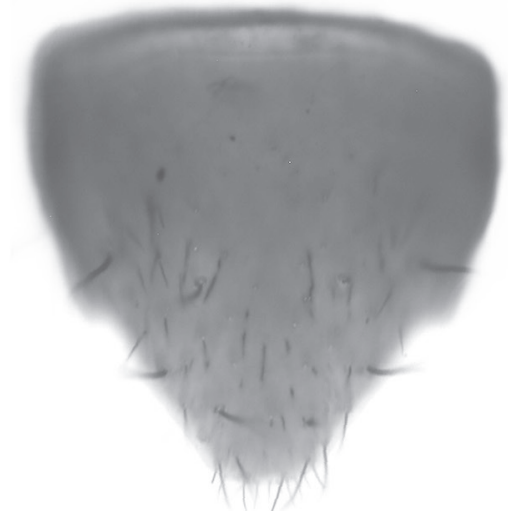

12
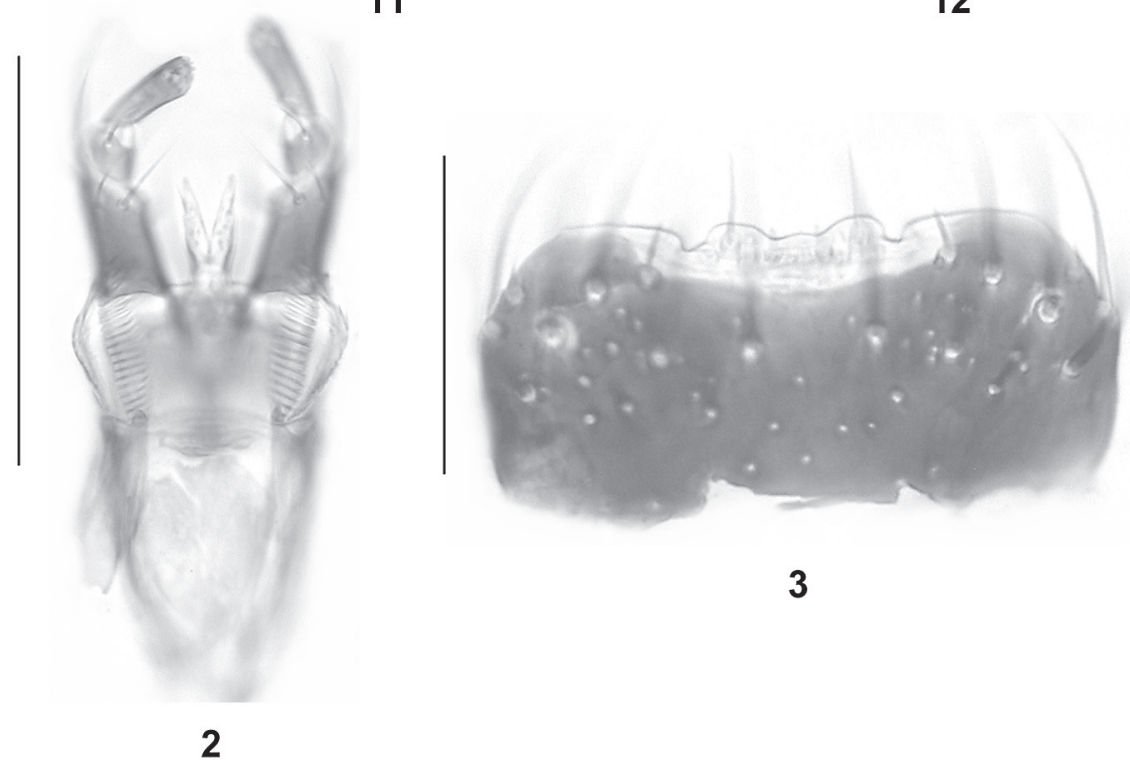

3

Figs 1-12: Anaulacaspsis nigra. Maxilla (1); labium (2); labrum (3); male tergite VIII (4-9); male sternite VIII (10-12). Scale bars: 1-2, 4-12: $0.1 \mathrm{~mm}$; 3: $0.05 \mathrm{~mm}$. 


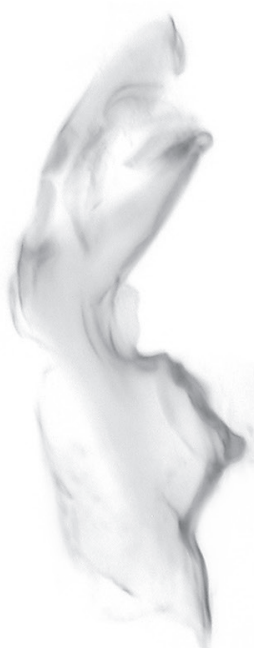

13

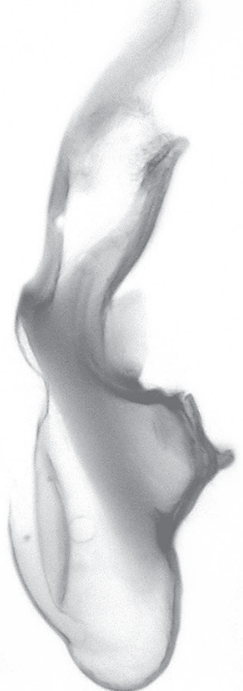

19
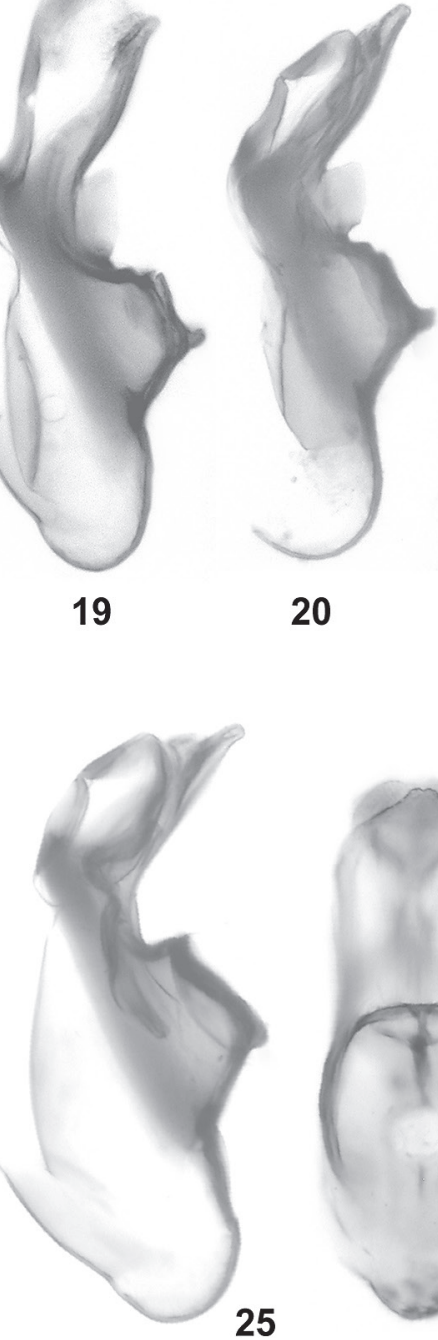

20

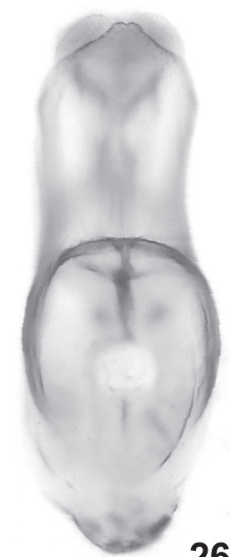

26

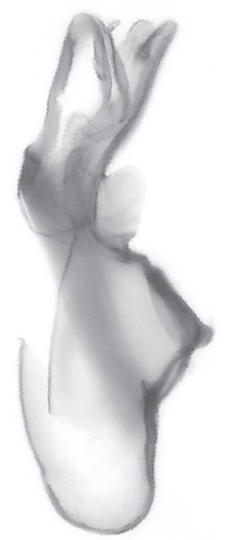

15

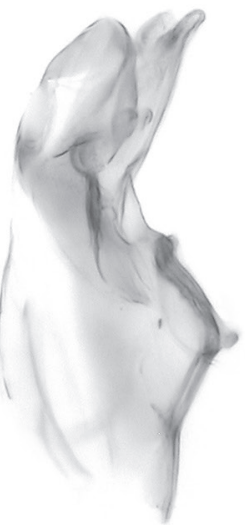

21

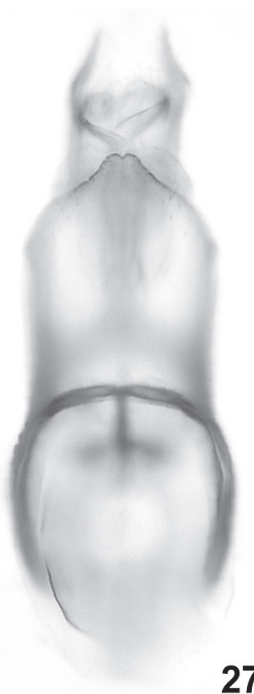

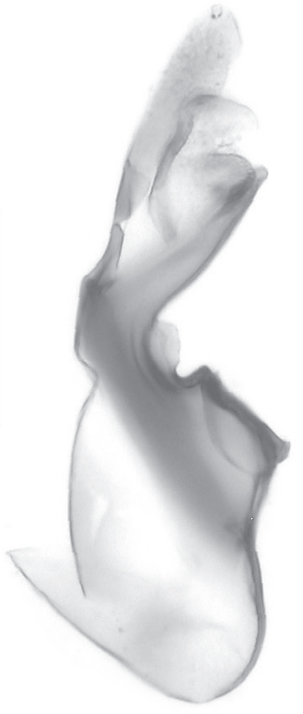

17

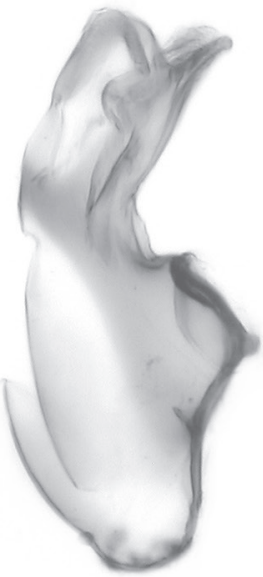

23

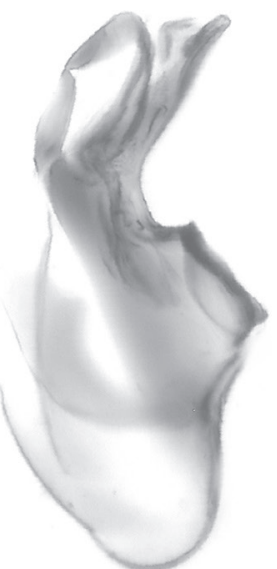

22

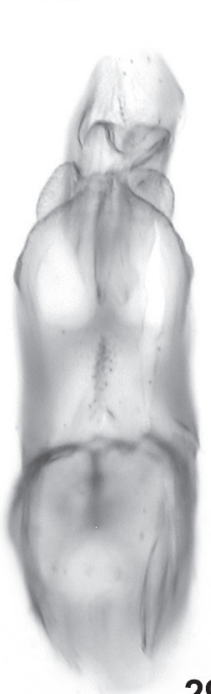

29

Figs 13-30: Anaulacaspsis nigra. Median lobe of aedeagus in lateral (13-25) and in ventral view (26-30) of the lectotype of $A$. nigra (13) and of males from Bosnia-Herzegovina (14), Bulgaria (15-16, 29), Ukraine (17, 30), mainland Greece (18), Turkey (19-20), Albania (21), Korfu $(22,28)$, and the Pelopónnisos (23-27). Scale bar: $0.1 \mathrm{~mm}$. 


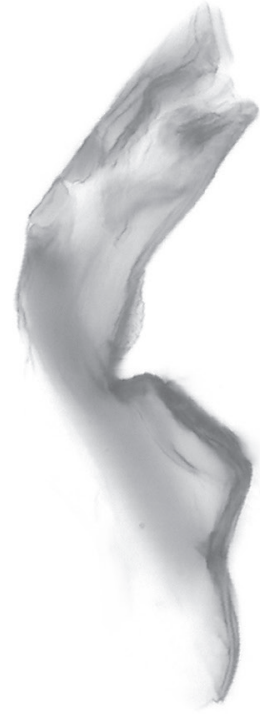

33

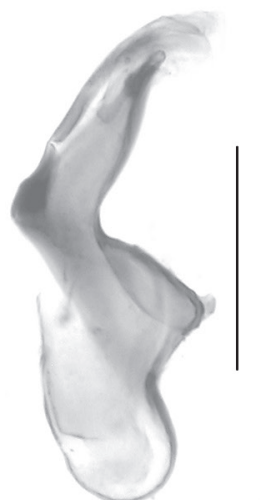

45

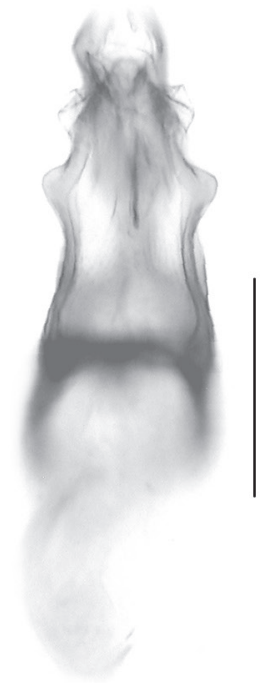

34

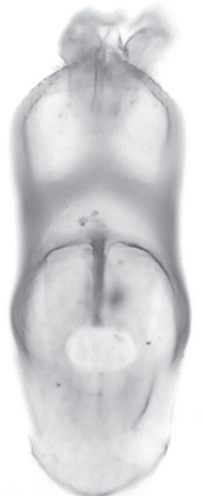

46

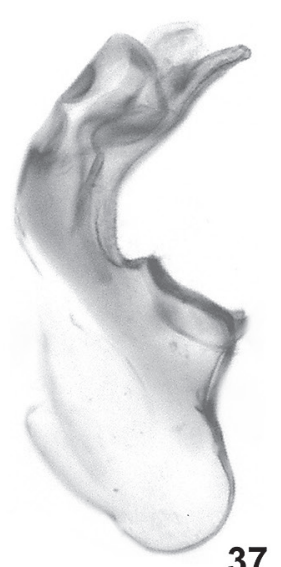

37

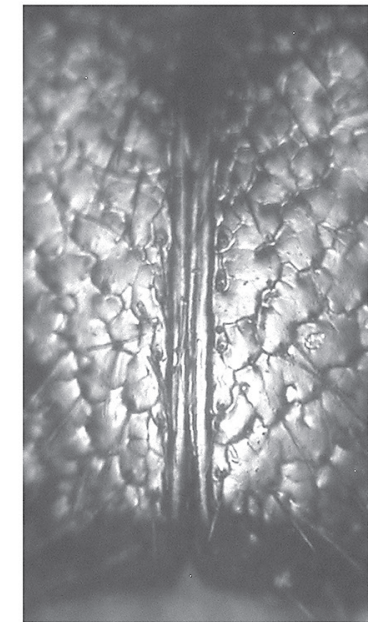

42

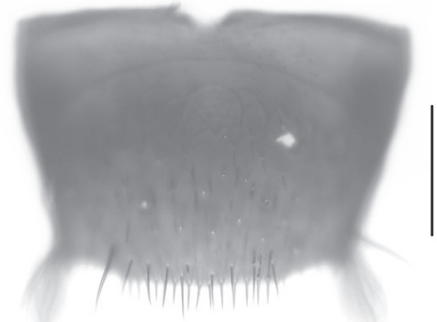

31

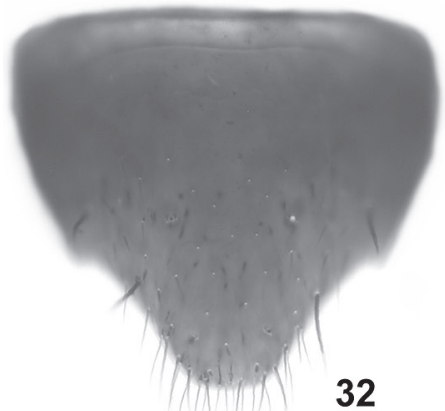

32

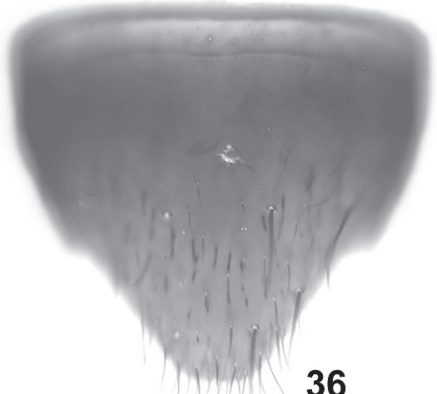

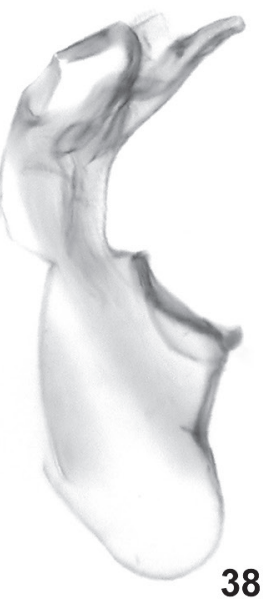
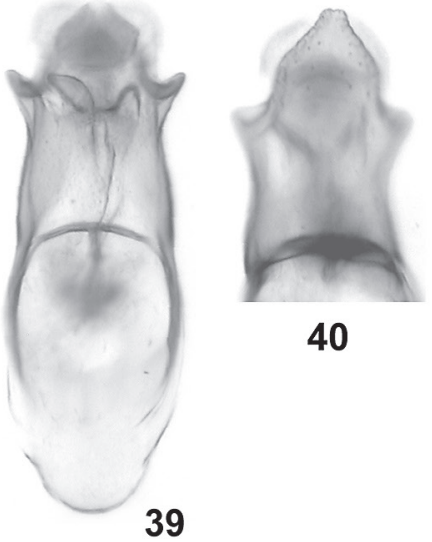

40

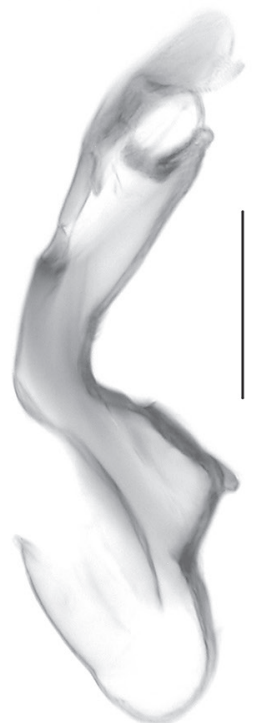

47

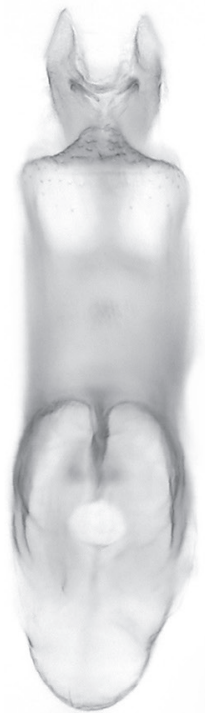

48

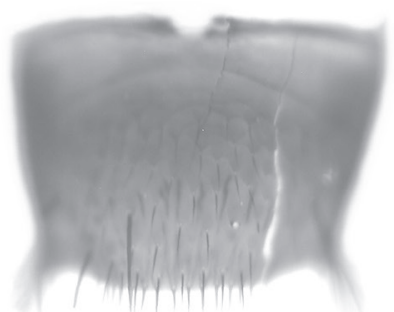

35

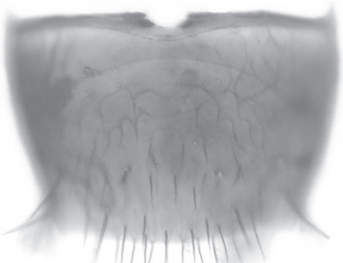

43

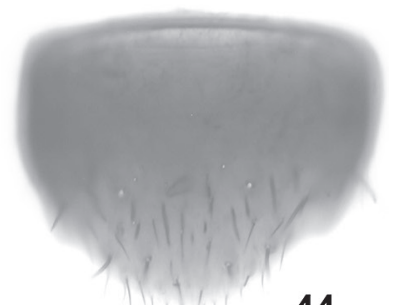

44

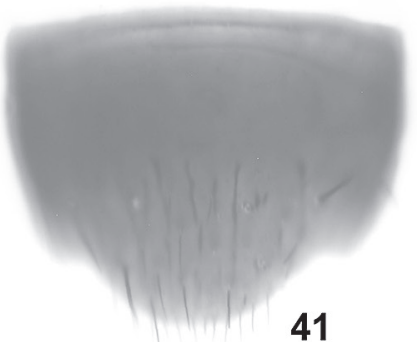

Figs 31-48: Anaulacaspsis caucasica (31-34), A. pseudonigra (35-41), A. reticulata (42-46), and A. libanotica (47-48): male tergite VIII $(31,35,43)$; male sternite VIII $(32,36,44)$; median lobe of aedeagus in lateral and in ventral view $(33-34,37-39,45-48)$; apical portion of median lobe in ventral view (40); female sternite VIII (41); sutural portion of elytra (42). Scale bars: $0.1 \mathrm{~mm}$. 

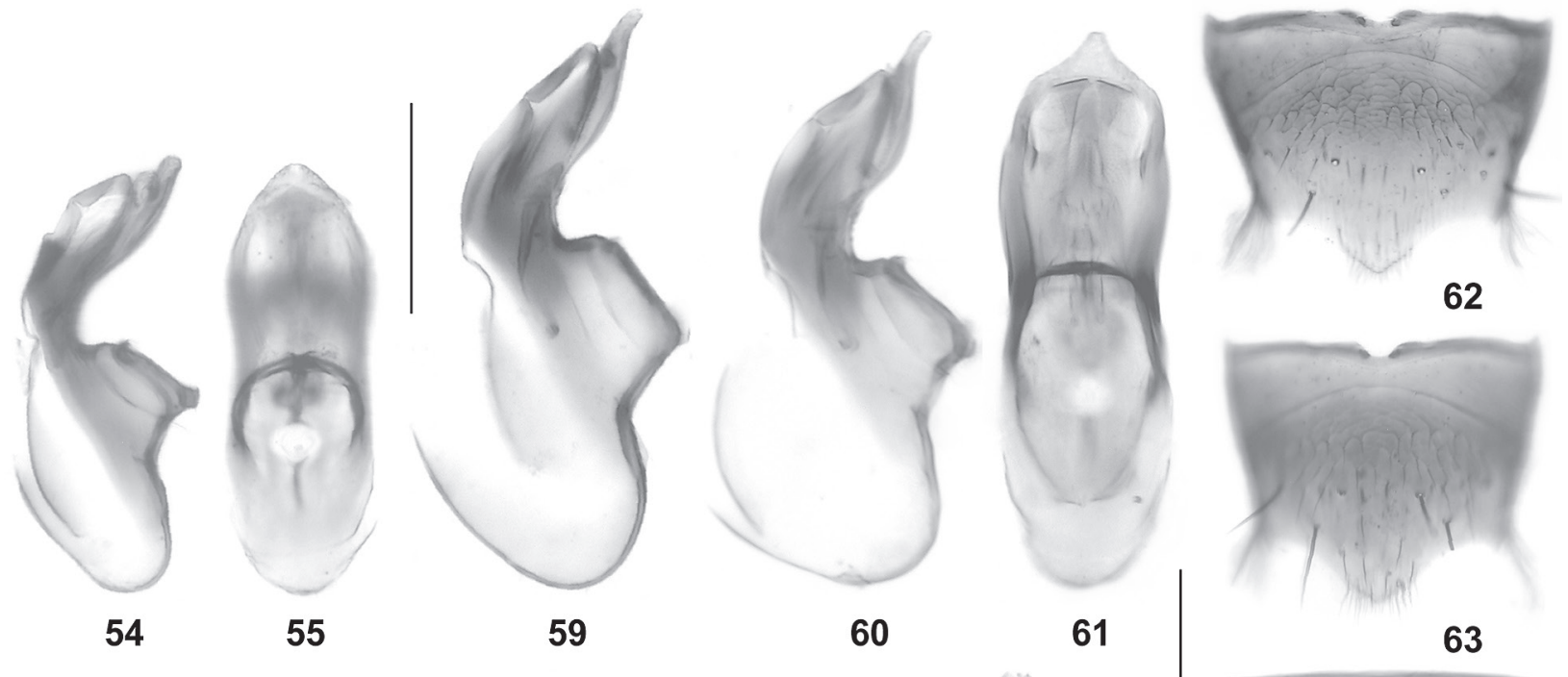

62
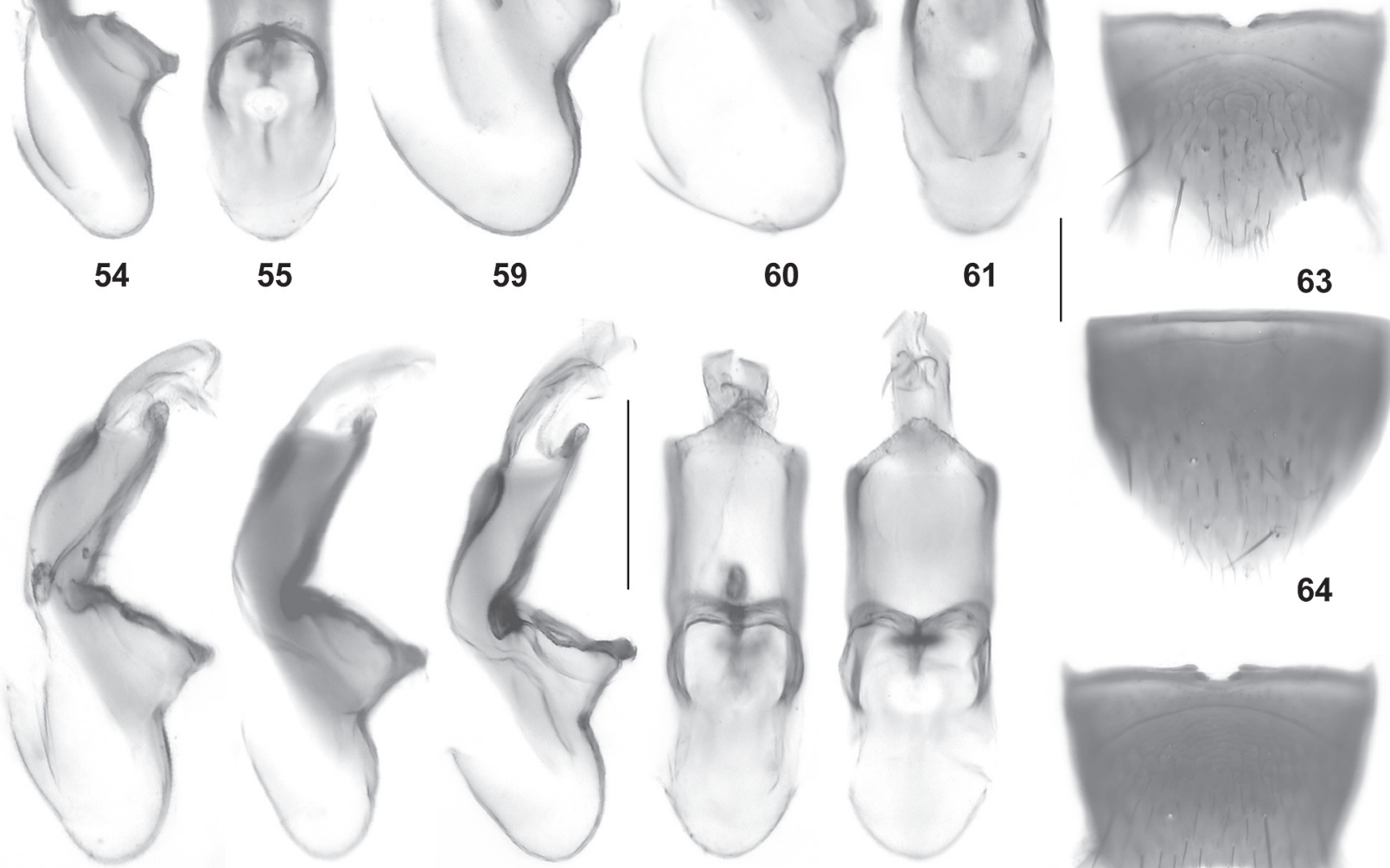

60

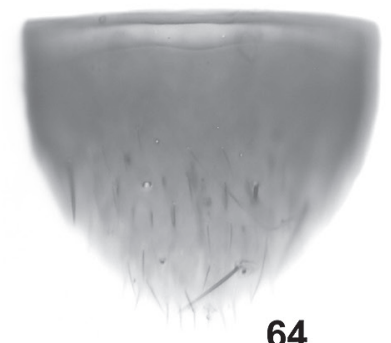

64

65

66

67
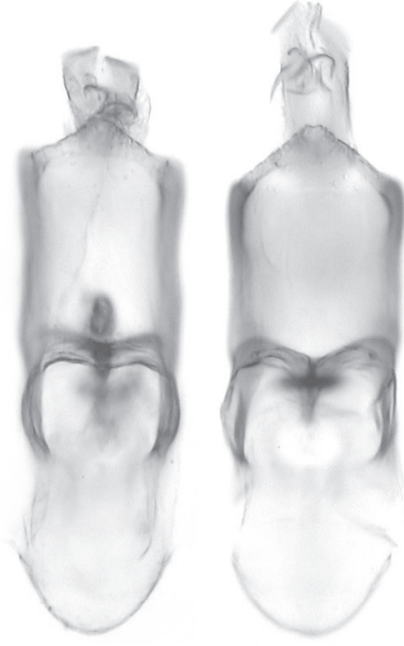

68

69

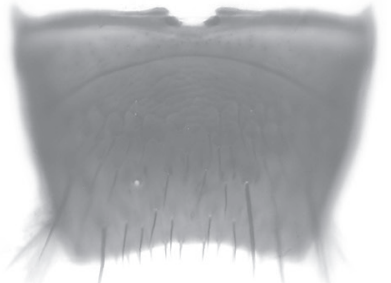

57

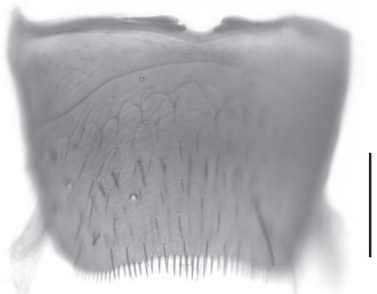

49

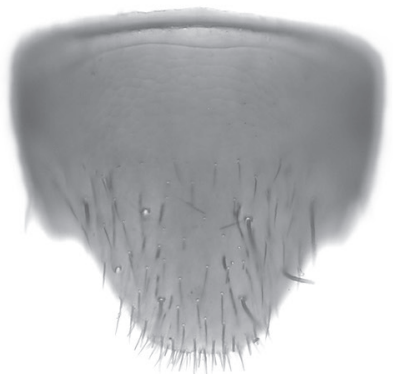

50

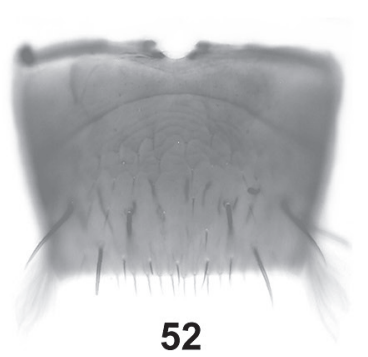

52

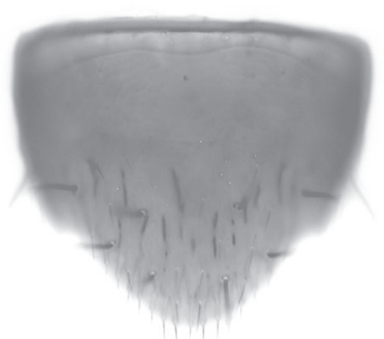

53

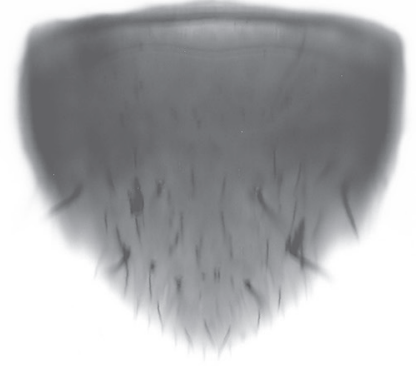

58

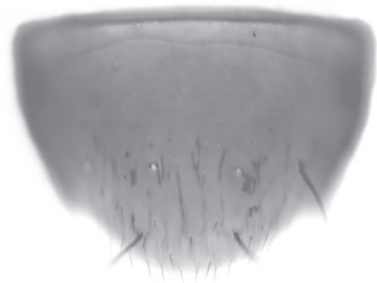

56

Figs 49-69: Anaulacaspsis libanotica (49-51), A. sinuata (52-56), A. nigerrima (57-61), and A. persica (62-69): male tergite VIII $(49,52,57,62-63)$; male sternite VIII $(50,53,58,64)$; female sternite VIII $(51,56)$; median lobe of aedeagus in lateral and in ventral view (54-55, 59-61, 65-69). Scale bars: $0.1 \mathrm{~mm}$. 


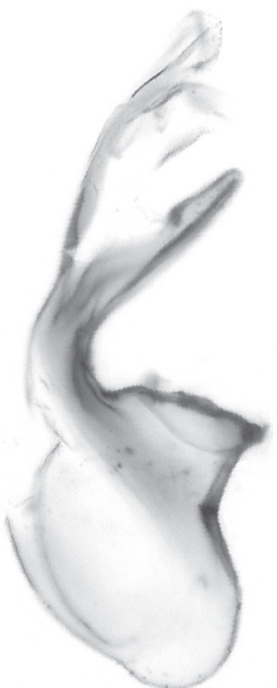

72

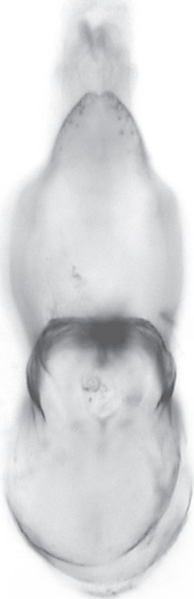

73

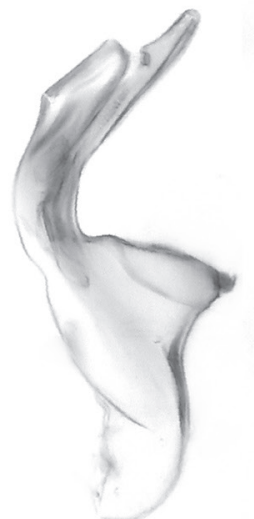

78

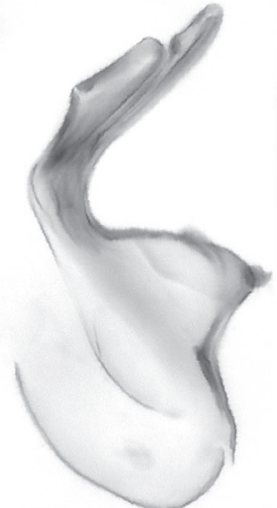

79

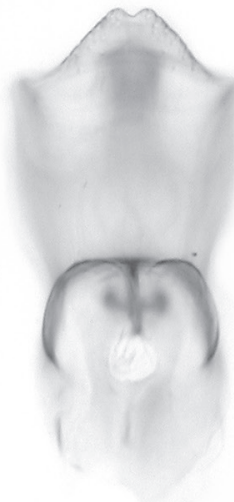

80

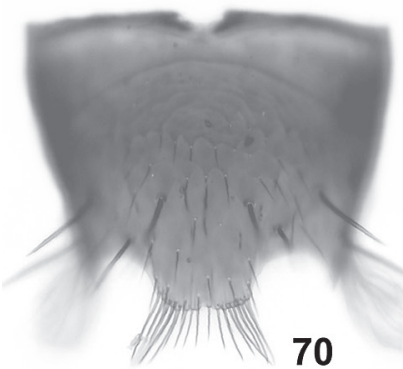

70

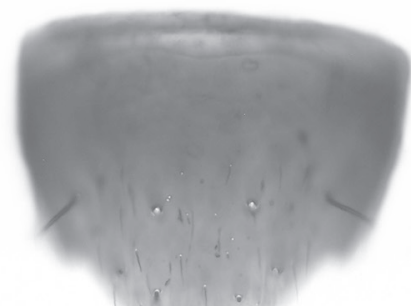

71

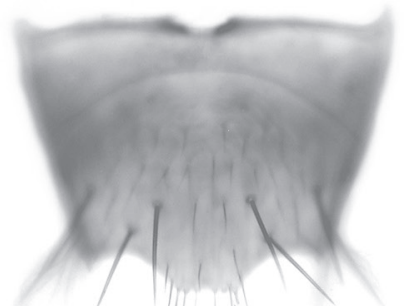

74

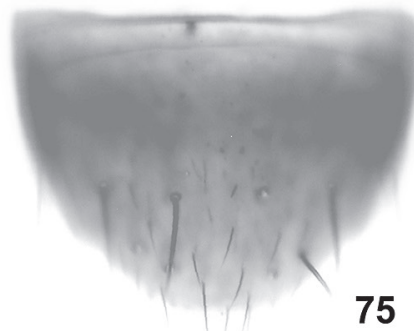

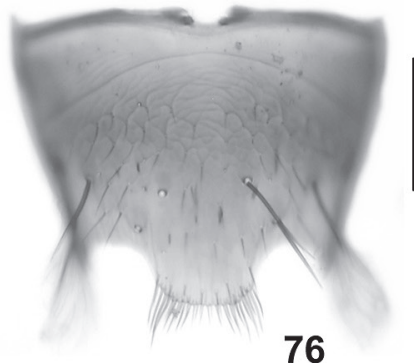

76
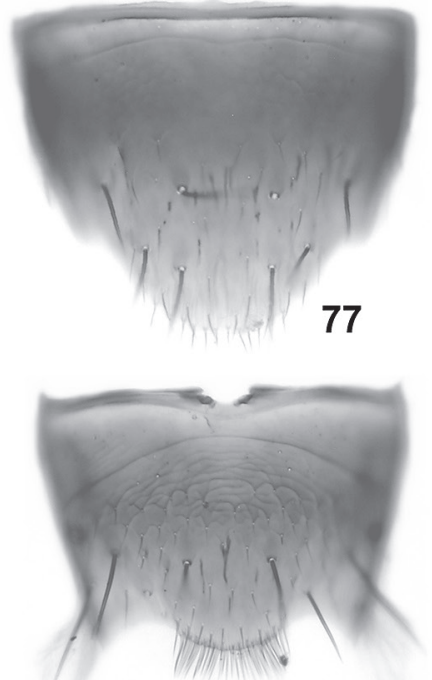

82

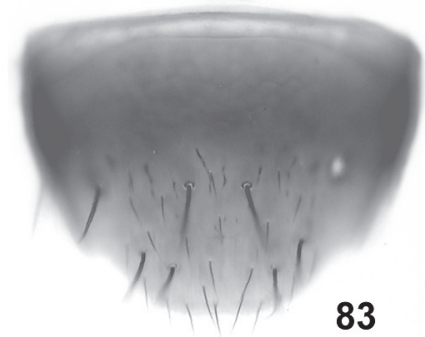

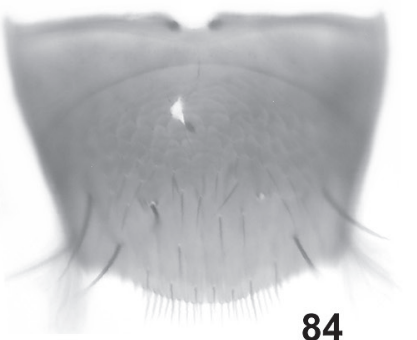

84
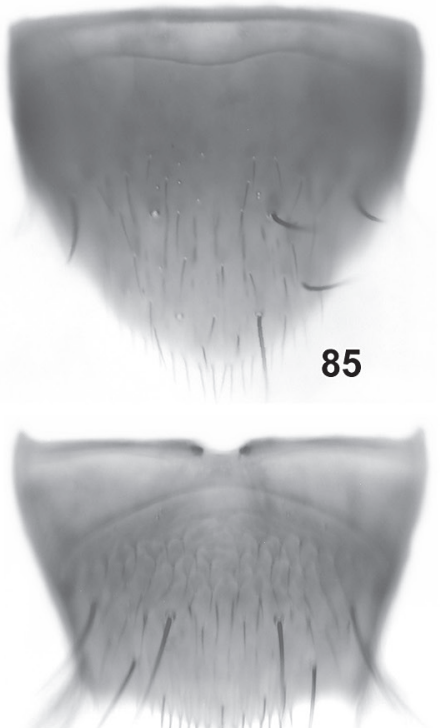

89

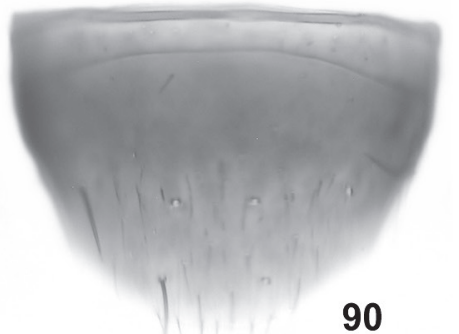

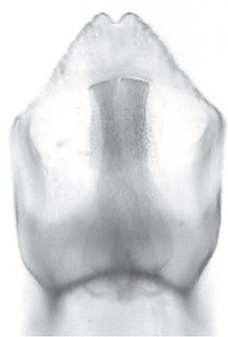

81
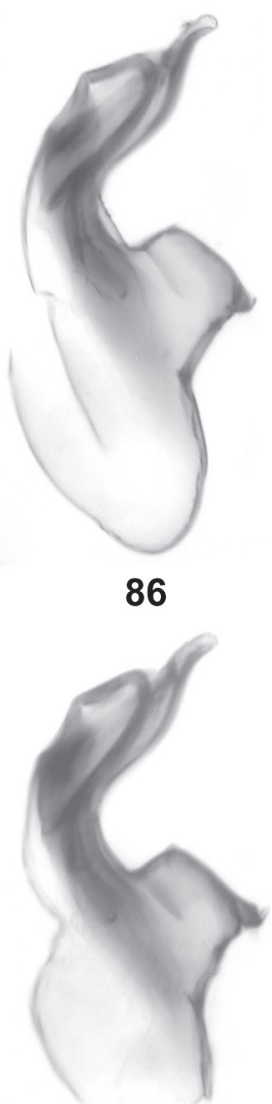

87

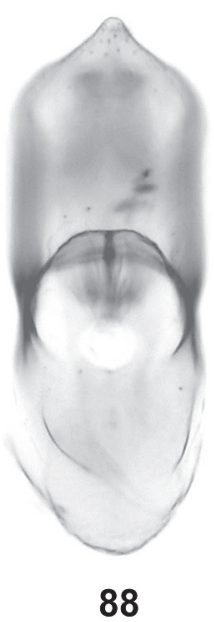

Figs 70-90: Anaulacaspsis truncata (70-75), A. convexa (76-83), and A. eminens (84-90): male tergite VIII (70, 76, 84); male sternite VIII $(71,77,85)$; median lobe of aedeagus in lateral and in ventral view $(72-73,78-80,86-88)$; female tergite VIII (74, 82, 89); female sternite VIII $(75,83,90)$; apical portion of aedeagus in ventral view $(81)$. Scale bars: $0.1 \mathrm{~mm}$. 

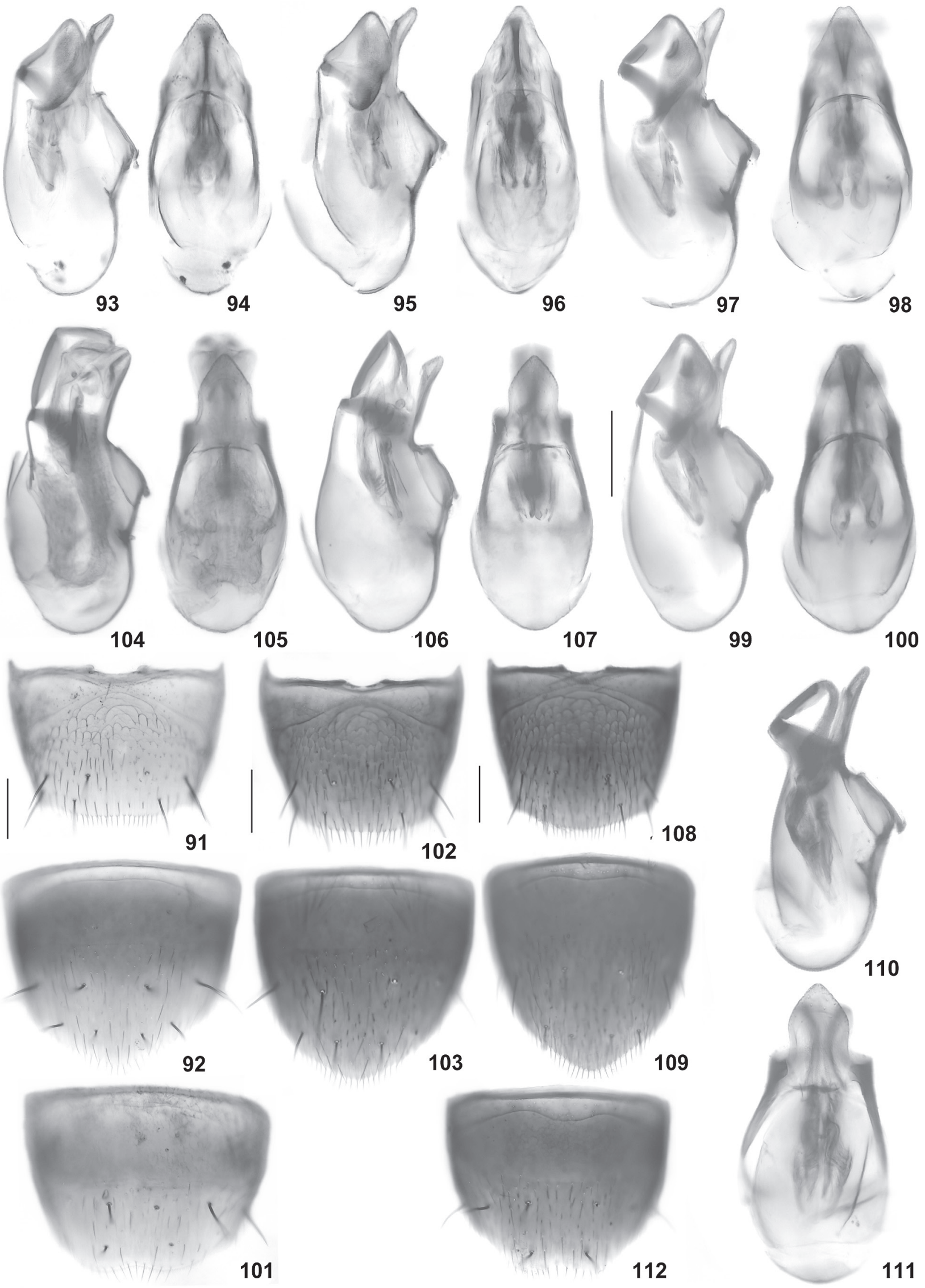

Figs 91-112: Anaulacaspsis naevula (91-101;93-94: Saudi Arabia;95-96: paralectotype; 97-98: Iran; 99-100: holotype of A. elegansides), A. desertorum (102-107), and A. seclusa (108-112): male tergite VIII (91, 102, 108); male sternite VIII (92, 103, 109); median lobe of aedeagus in lateral and in ventral view (93-100, 104-107, 110-111); female sternite VIII (101, 112). Scale bars: $0.1 \mathrm{~mm}$. 


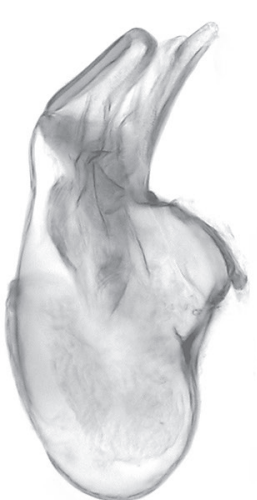

115

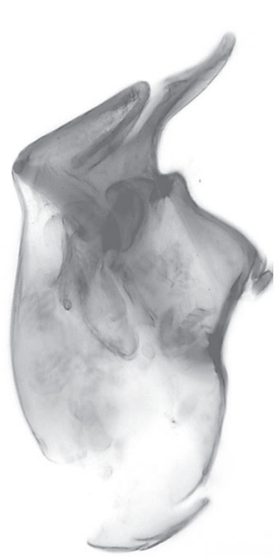

123

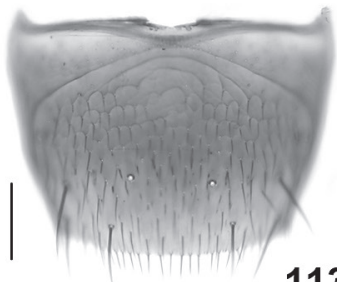

113

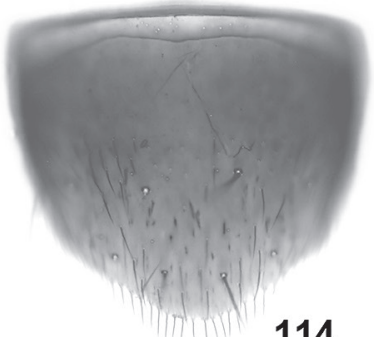

114

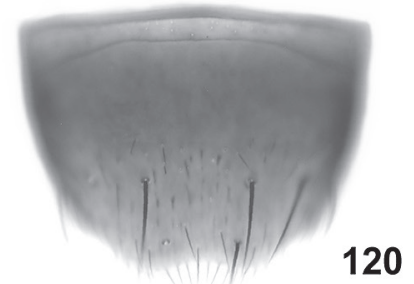

124

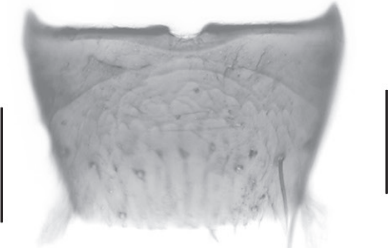

128
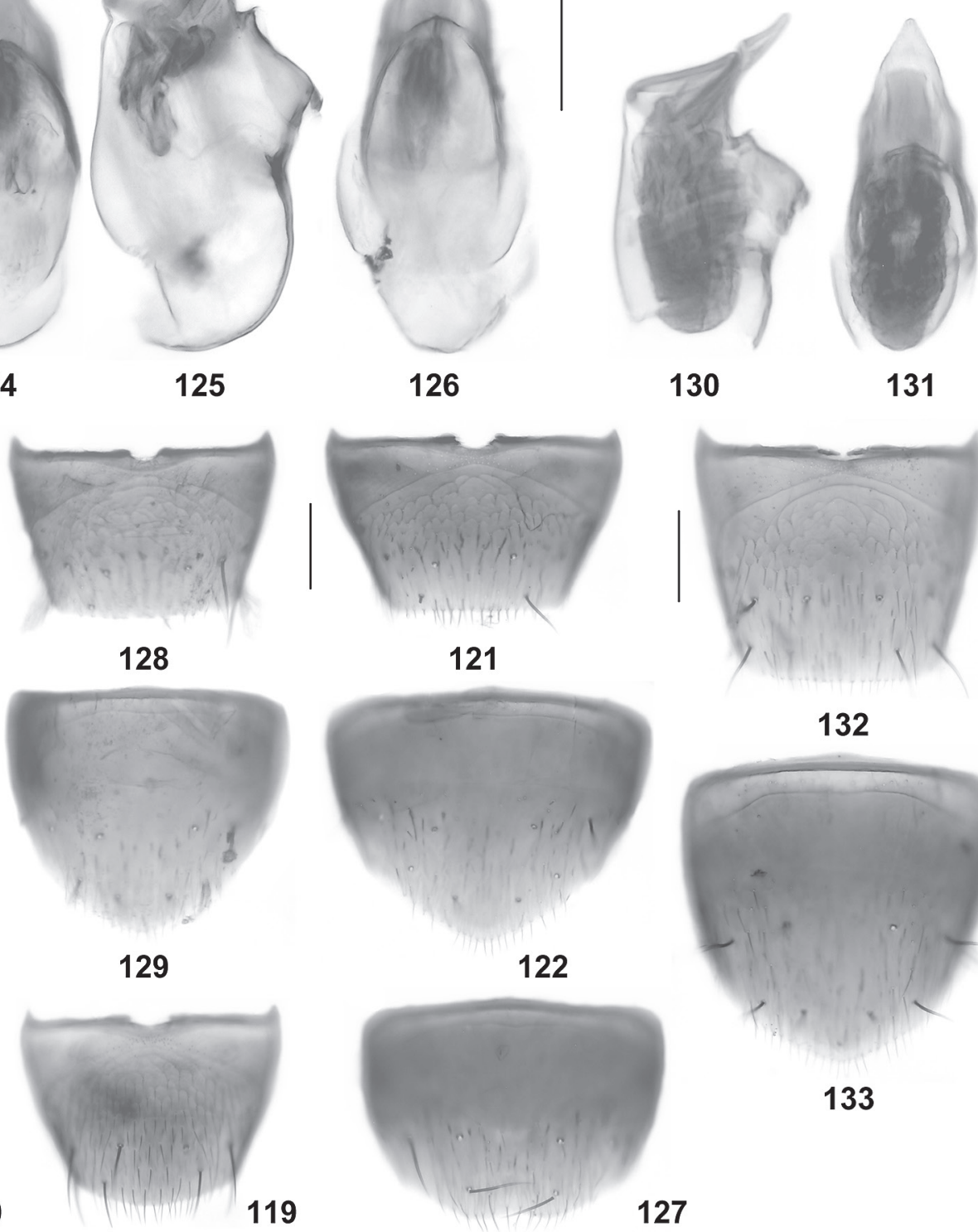

126

130

131

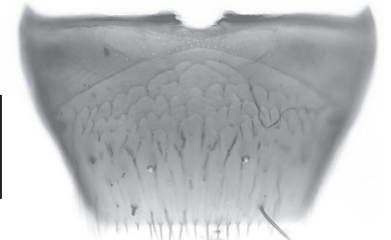

121

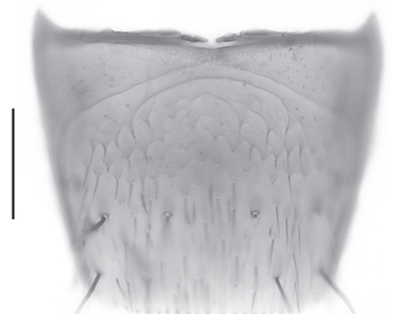

132

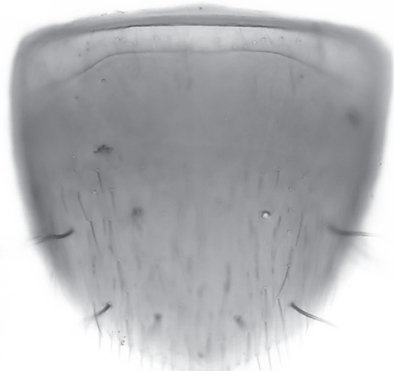

133

Figs 113-135: Anaulacaspsis seclusa (113-120; 115-116: holotype of A. klapperichi; 113-114, 117-120: Iran), A. gratilla (121-131; 121-124: lectotype of A. lutzi; 128-131: nanistic male from Azerbaijan), and A. cristata (132-135): male tergite VIII (113, 121, 128, 132); male sternite VIII $(114,122,129,133)$; median lobe of aedeagus in lateral and in ventral view $(115-118,123-126,130-131$, 134-135); female tergite VIII (119); female sternite VIII $(120,127)$. Scale bars: $0.1 \mathrm{~mm}$. 


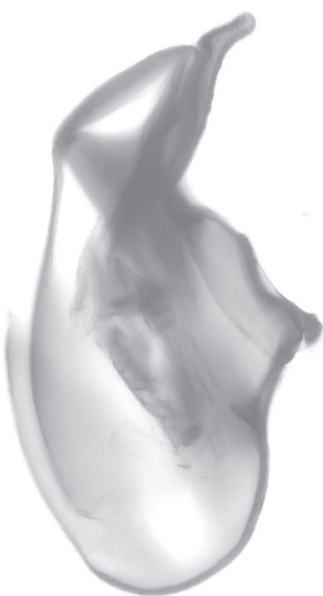

138

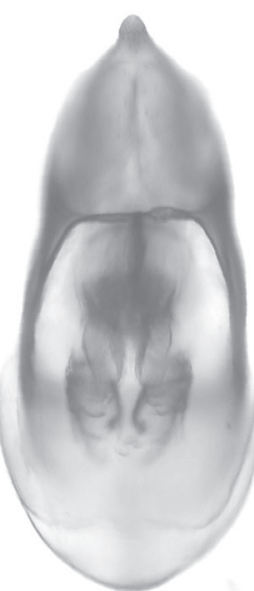

139

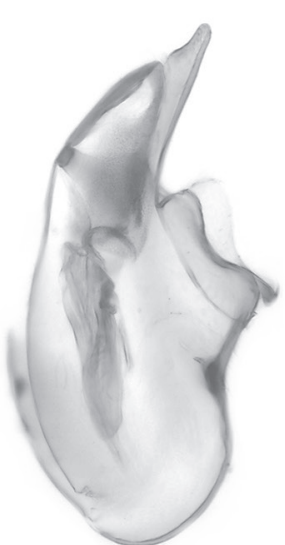

144

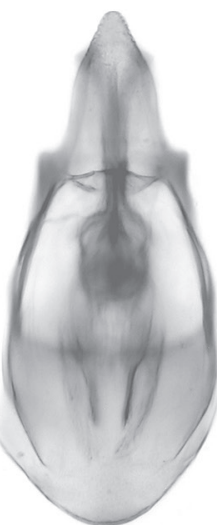

145

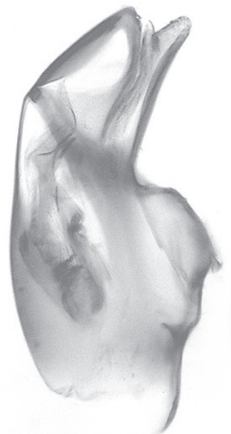

148

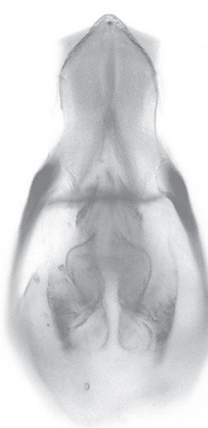

149

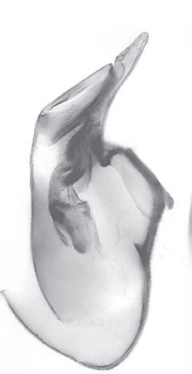

153

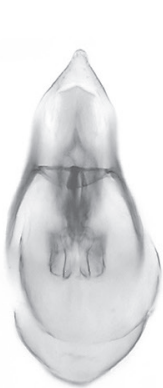

154

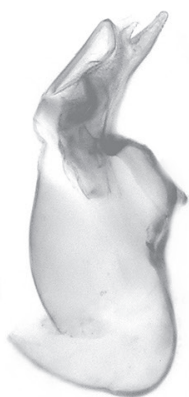

155

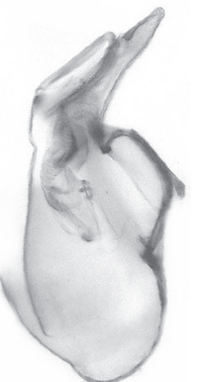

156
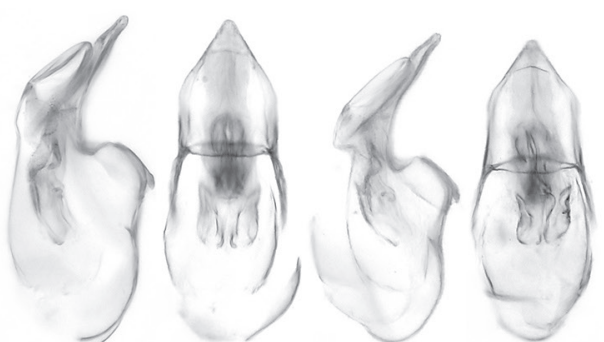

159

160

161

162
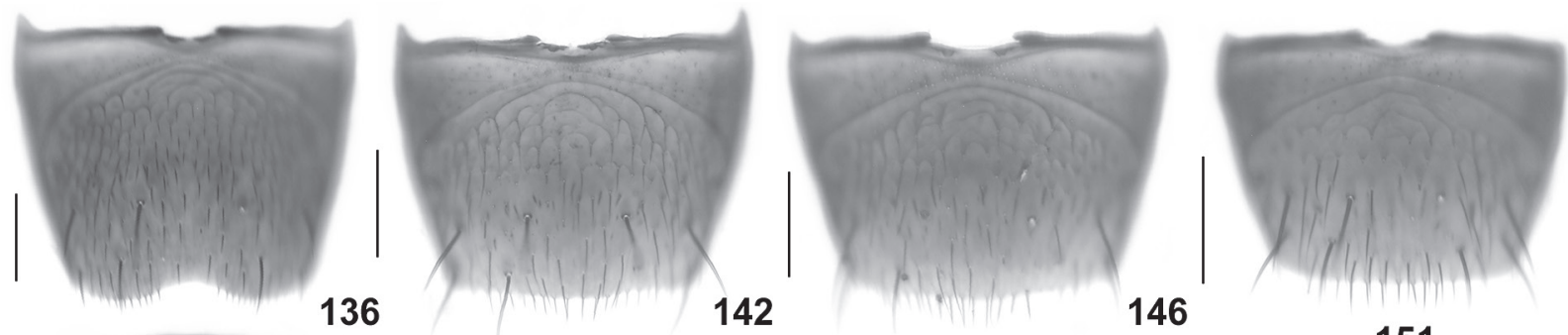

151
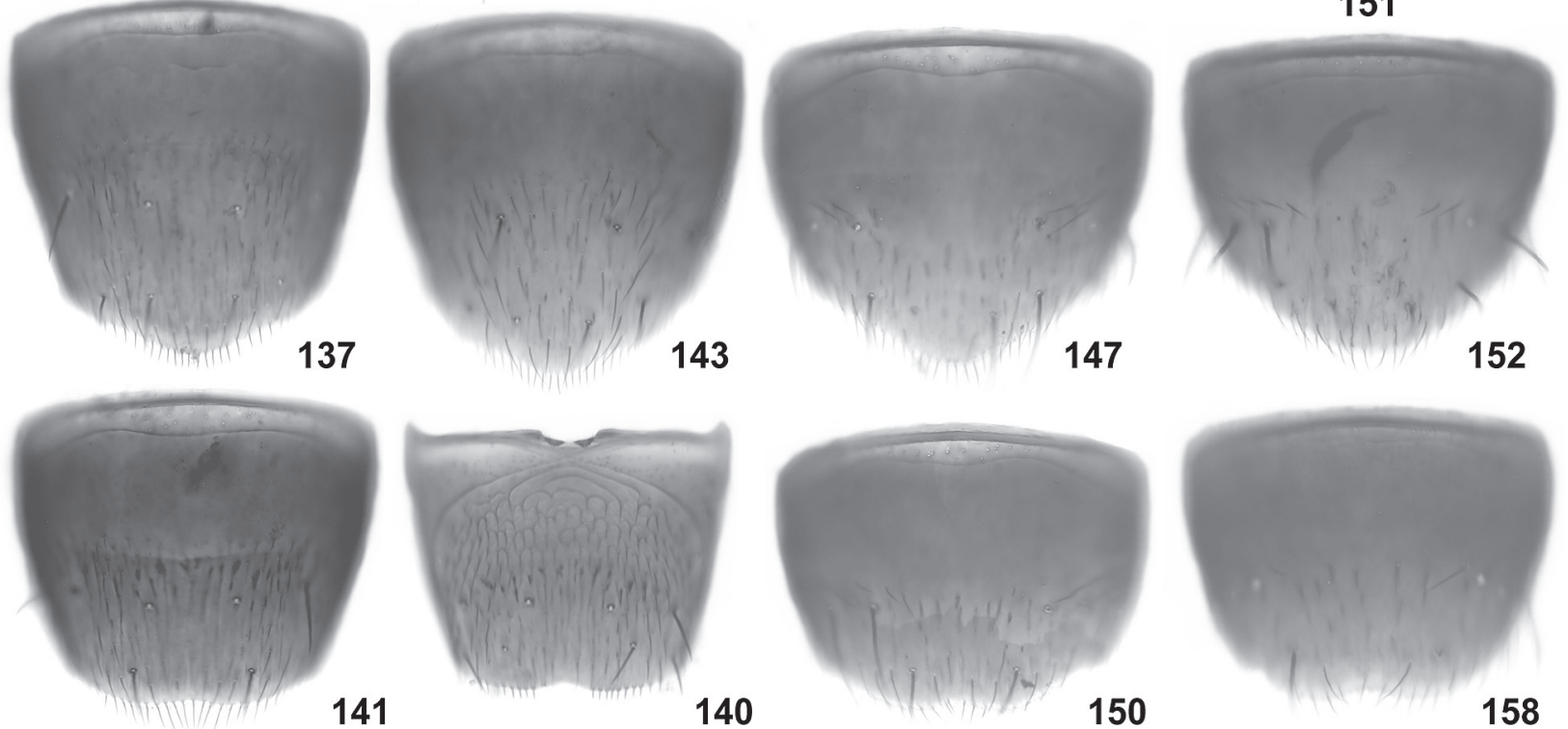

Figs 136-163: Anaulacaspsis excisa (136-141), A.pamphylica (142-145), A. flavomarginata (146-150), A. laevigata (151-158; 153-154: lectotype; 155: Pelopónnisos; 156: Lesbos), and A. iberica (159-163): male tergite VIII (136, 142, 146, 151); male sternite VIII $(137,143,147,152)$; median lobe of aedeagus in lateral and in ventral view (138-139, 144-145, 148-149, 153-156, 159-162); female sternite VIII (141, 150, 158); female tergite VIII (140); apex of ventral process of aedeagus in lateral view (157, 163). Scale bars: $0.1 \mathrm{~mm}$. 


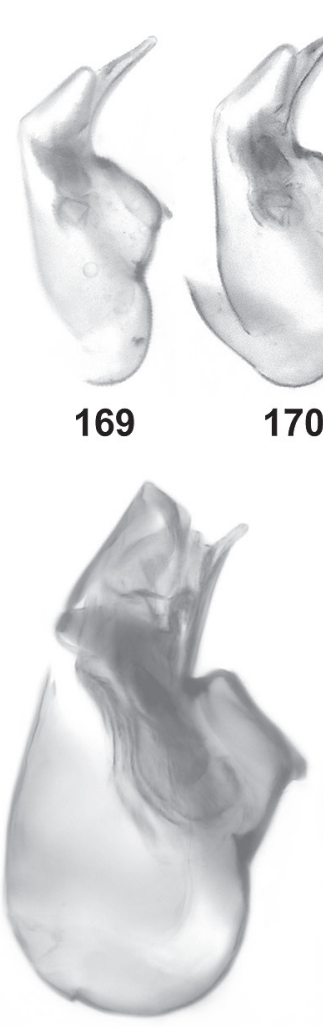

175
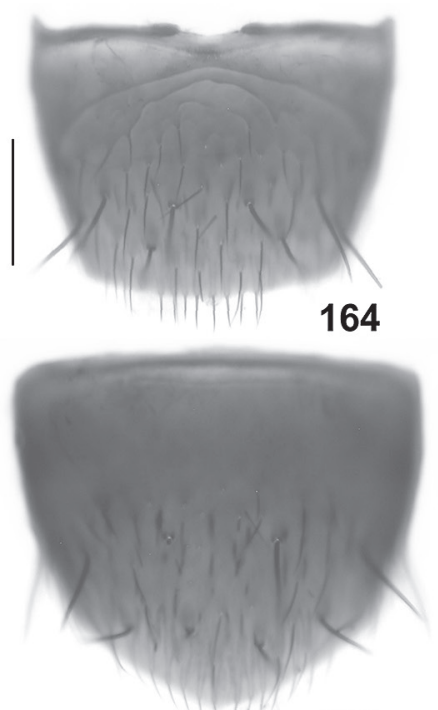

165
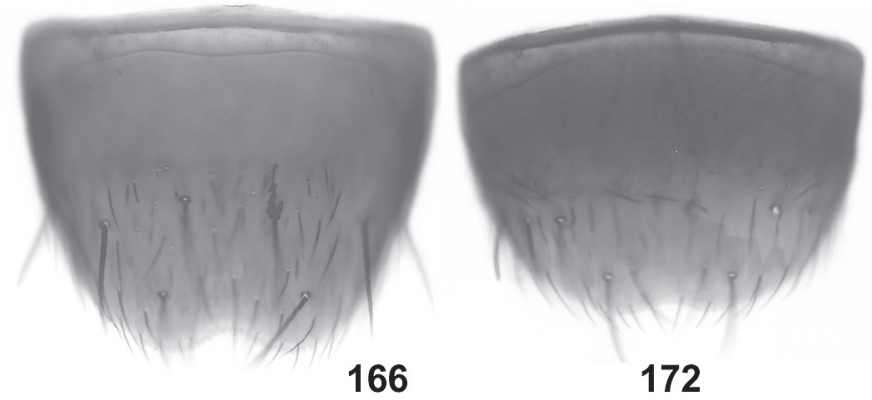

168

167

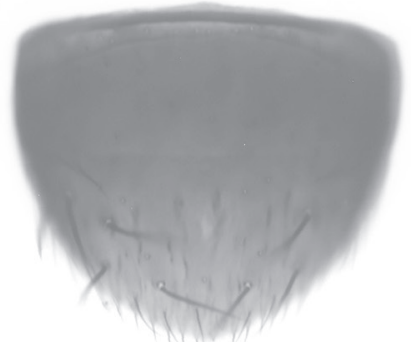

Figs 164-186: Anaulacaspsis iberica (164-166), A. nigrina (167-172), A. beijingensis (173-177), A. taiwanica (178-183), and A. gilva (184-186): male tergite VIII $(164,167,173,178,184)$; male sternite VIII $(165,168,174,179,185)$; female sternite VIII $(166,172,177,183)$; median lobe of aedeagus in lateral and in ventral view (169-171, 175-176, 180-181); female tergite VIII (182); spermatheca (186). Scale bars: $0.1 \mathrm{~mm}$. 


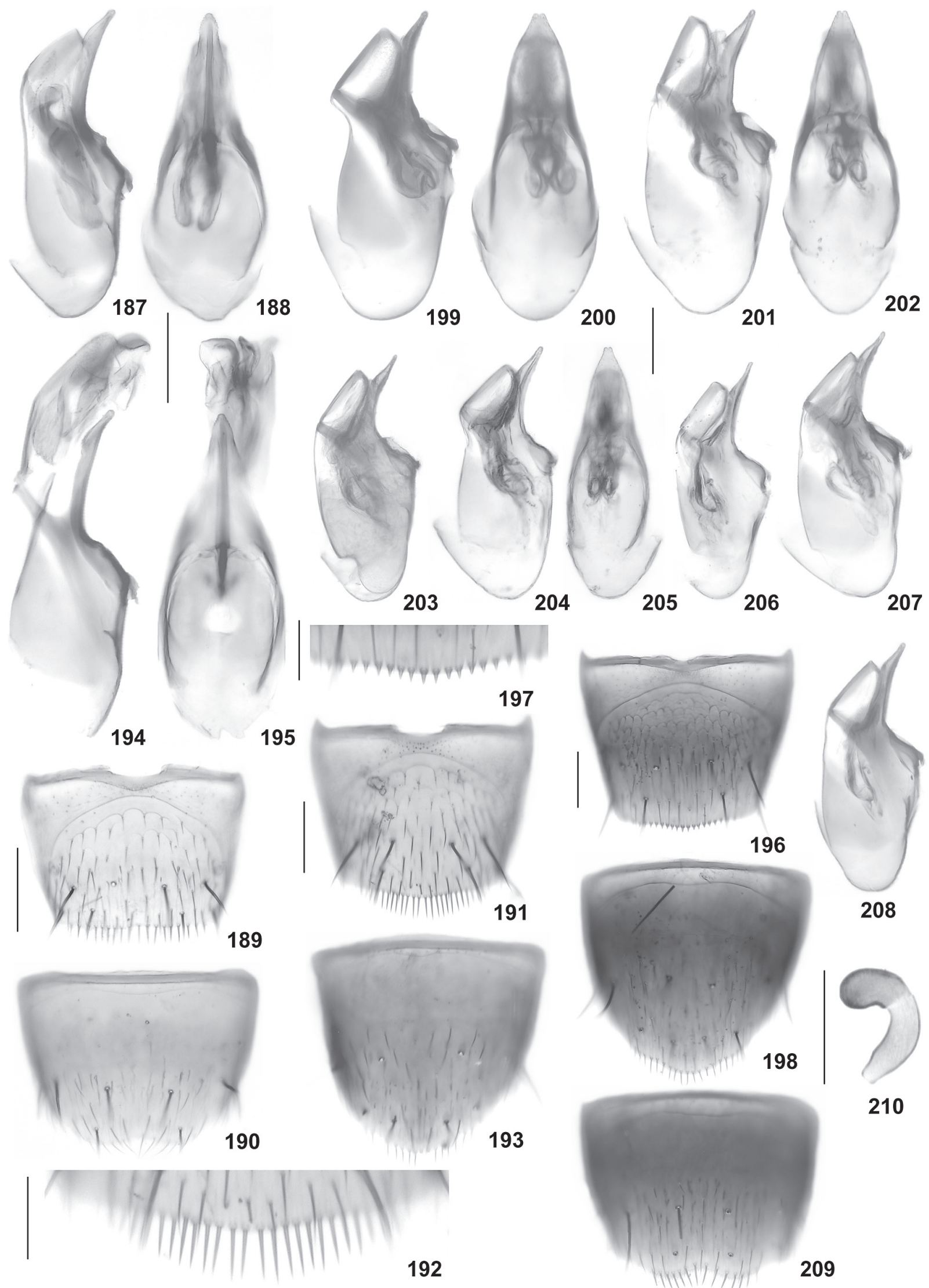

Figs 187-210: Anaulacaspsis gilva (187-190), A.pectinata (191-195), and A.formosa (196-210; 199-200: Tunisia; 201-203: Morocco; 204-205: holotype of A. macra; 206: Murcia; 207-208: Andalucía): median lobe of aedeagus in lateral and in ventral view (187-188, 194-195, 199-208); female tergite VIII (189); female sternite VIII (190, 209); male tergite VIII (191, 196); posterior margin of male tergite VIII $(192,197)$; male sternite VIII $(193,198)$; spermatheca (210). Scale bars: 187-191, 193-196, 198-210: $0.1 \mathrm{~mm} ; 192,197: 0.05 \mathrm{~mm}$. 

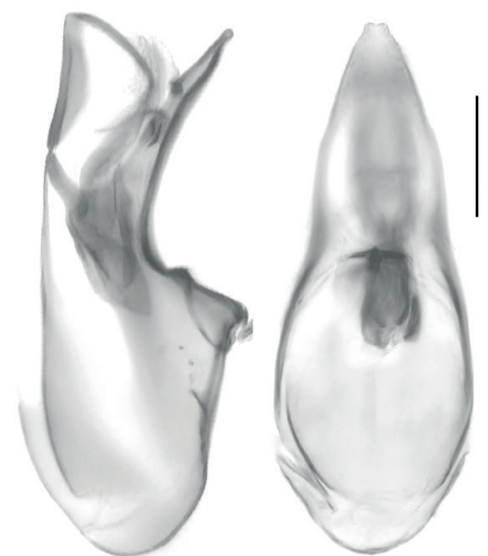

214

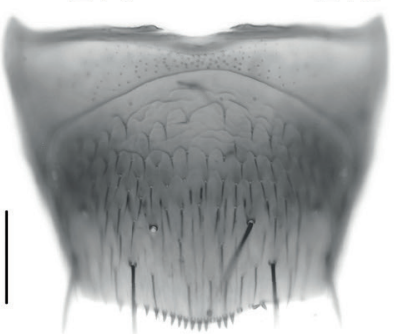

212
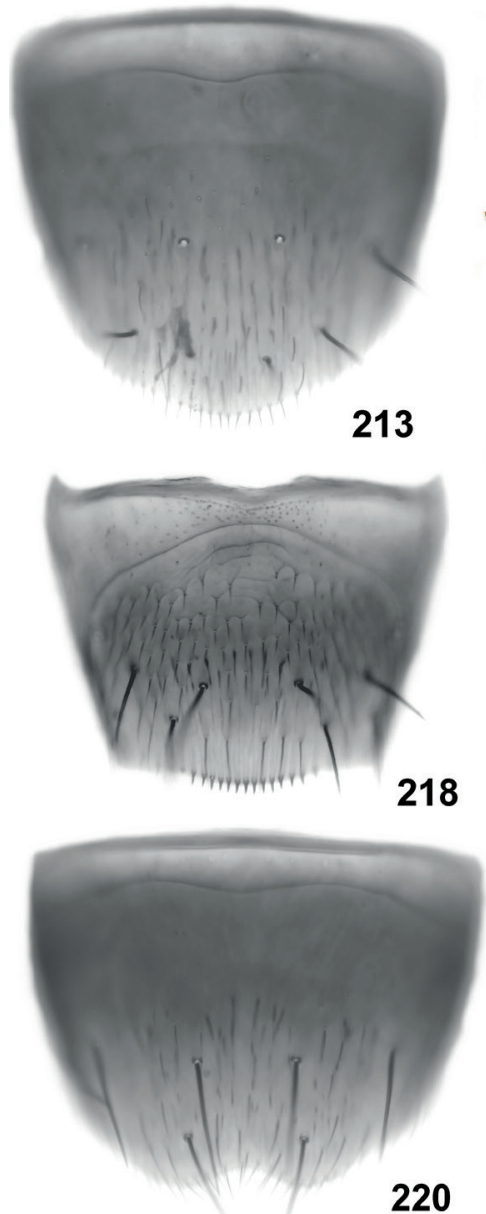

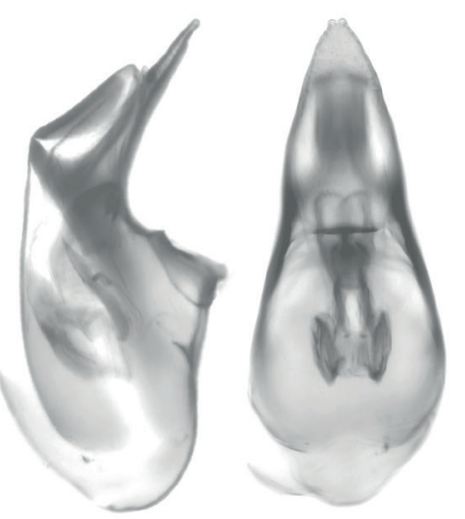

216

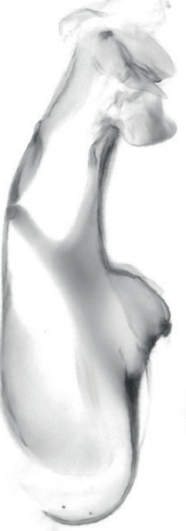

224

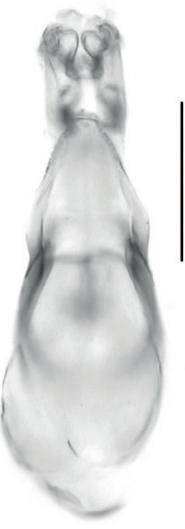

225

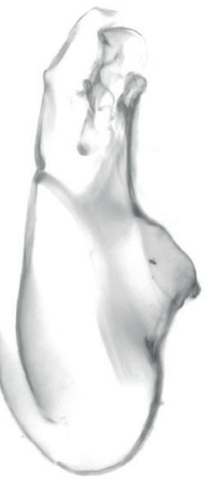

226
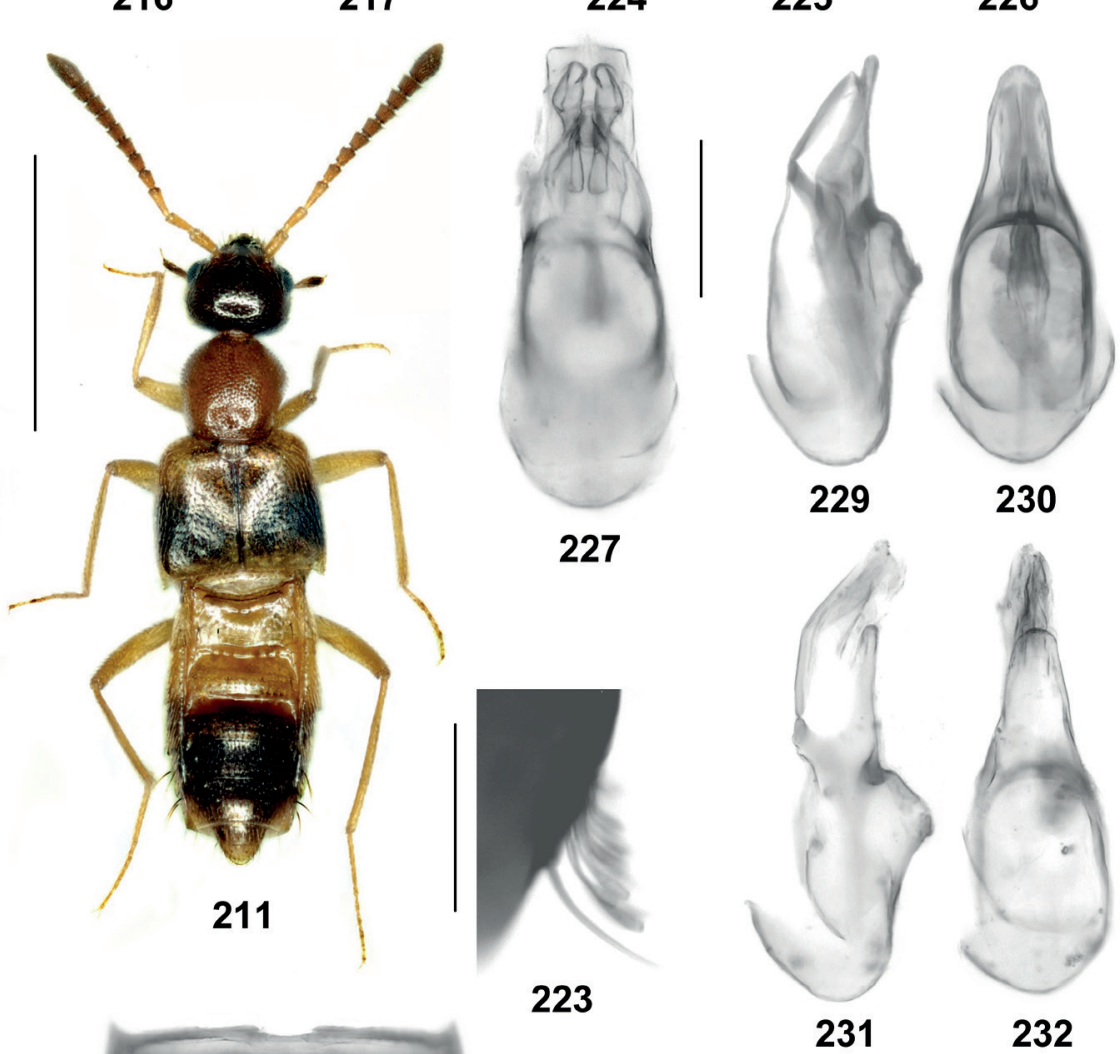

223

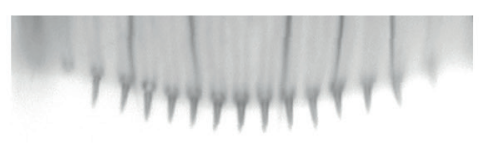

219

221

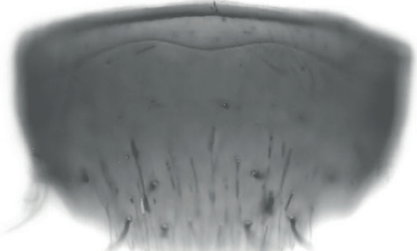

222

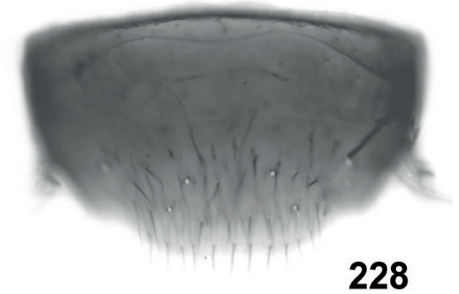

Figs 211-232: Anaulacaspsis formosa (211), A. elegans (212-220), A. beesoni (221-228; 224-225: lectotype), and A. inexpectata (229-232; 231-232: holotype): habitus (211; photo by Marc Tronquet); male tergite VIII (212, 221); male sternite VIII (213, 222); median lobe of aedeagus in lateral and in ventral view (214-217, 224-227, 229-232); female tergite VIII (218); posterior margin of female tergite VIII (219); female sternite VIII (220, 228); postero-lateral portion of sternite VIII (223). Scale bars: $211: 1.0 \mathrm{~mm}$; 212-218, 220-222, 224-232: 0.1 mm; 219, 223: $0.05 \mathrm{~mm}$. 


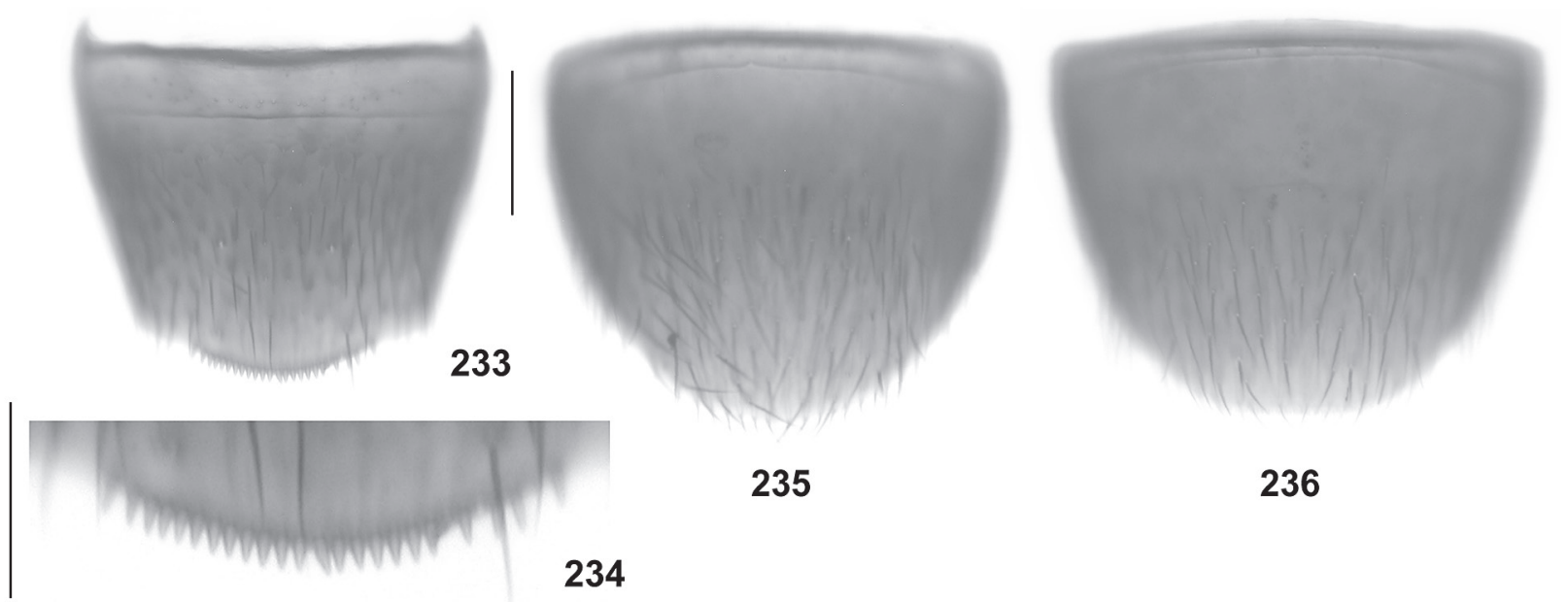

Figs 233-236: Anaulacaspsis inexpectata: male tergite VIII (233); posterior margin of male tergite VIII (234); male sternite VIII (235); female sternite VIII (236). Scale bars: 233, 235-236: $0.1 \mathrm{~mm}$; 234: $0.05 \mathrm{~mm}$.

Comment: The original description of Aleochara nigra is based on an unspecified number of syntypes in "Mus. Prof. Knoch" (Gravenhorst 1802). The locality is not specified, but the species was described in a work on the Staphylinidae of the environs of Braunschweig (North Germany). Six syntypes, two males and four females, were found in the collections of the MNB. One of the males is designated as the lectotype.

Aside from the type series of A. nigra, four pins with syntype labels of Aleochara picea, two of them with mounting cards with only a single leg left, were located in the MNB. Two syntypes, a male and a female, are still in good condition. The male is designated as the lectotype. The previously established synonymy of A. picea with A. nigra is confirmed.

Bernhauer (1910) described Falagria nigra var. jonica based on an unspecified number of syntypes from "Korfu, Attika und Kroatien", stating that this variety was distinguished from Central European populations by longer and broader elytra, but otherwise identical. FAGEL (1969) treated this taxon as a species, designated a female [sic] from "Attica" from the Bernhauer collection as the lectotype, recorded A. jonica from "différentes parties de la Grèce, de Dalmatie et ... Turquie d'Europe : Istanbul (Rumeli Hisar)", and assigned the material studied from Corfu to a new species. A comparison of the lectotype of $A$. jonica with material from other regions studied in the course of the present revision, however, revealed that, regarding its external and the female secondary sexual characters, the specimen is within the range of intraspecific variation of A. nigra. Moreover, A. nigra appears to be the only species of the A. nigra group present in mainland Greece. Consequently, A. jonica is placed in synonymy with $A$. nigra.

The original description of Melagria anatolica is based on a male holotype from "Alanya (plage)", one female from the same locality, one female of "même origine, sur plages du Dim Irmak", and two females from the Rhodopes in Bulgaria (FAGEL 1969). An examination of material from various regions revealed that the type material of
M. anatolica is conspecific with that of $A$. nigra (see notes on intraspecific variation below).

Melagria corcyrana was described from a male holotype and seven paratypes from "Corfu" (FAgEL 1969). Material from Corfu differs from many other populations of A. nigra by the modifications of the male pronotum (with an impression in posterior half rather than with a median sulcus) and by the absence of a pronounced semi-transparent carina at the base of the ventral process. However, similarly deviant character conditions were observed also in other populations, e.g. from the Pelopónnisos and Albania. Moreover, these differences do not correspond to a plausible distribution pattern, so that they are interpreted as intraspecific variation of $A$. nigra.

Additional material examined: Spain: Cataluña: $10^{*}$, Olot $\left[42^{\circ} 11^{\prime} \mathrm{N}, 2^{\circ} 28^{\prime} \mathrm{E}\right]$ env., pitfall, 14.VII.1977, leg. Hoffmann (MHNG).

France: Aquitaine: 19 , Gironde, Lamothe $\left[44^{\circ} 37^{\prime} \mathrm{N}\right.$, $\left.0^{\circ} 59^{\prime} \mathrm{W}\right]$, 8.IX.1928, leg. Tempère (MHNG); 1 \% , Léognan [44.43' $\left.\mathrm{N}, 0^{\circ} 35^{\prime} \mathrm{W}\right]$, leg. Gouin (MHNG); 1 오․ Beautiran [ $44^{\circ} 41^{\prime} \mathrm{N}, 0^{\circ} 26^{\prime} \mathrm{W}$ ], VII.1944 (MHNG); 1 ․ Gironde, Gajac, $\left.44^{\circ} 26^{\prime} \mathrm{N}, 0^{\circ} 08^{\prime} \mathrm{W}\right]$, 28.VI.1908 (MHNG). Midi-Pyrénées: $10^{\star}, 2$ 우, Hautes-Pyrénées, Luz-Saint-Sauveur ["Luz"; 42 $52^{\prime} \mathrm{N}, 0^{\circ} 00^{\prime} \mathrm{E}$ ], IX.1911, leg. Tempère (MHNG, cAss). Picardie: 1 ㅇ, Becquigny $\left[50^{\circ} 00^{\prime} \mathrm{N}, 3^{\circ} 27^{\prime} \mathrm{E}\right]$, near Bohain-en-Vermandois, 10.-19. IX.1918, leg. Salchert (MNB). Alsace: 1 우 Strasbourg, VIII.1953 (MHNG). Rhône-Alpes: $10^{7}$, Megève [45 $51^{\circ} \mathrm{N}, 6^{\circ} 36^{\prime} \mathrm{E}$ ], X.1960 (MHNG). Provence: $10^{\circ}$, Var, Agay $\left[43^{\circ} 26^{\prime} \mathrm{N}, 6^{\circ} 52^{\prime} \mathrm{E}\right]$, 30.I.1955 (MHNG); $10^{\circ}$, Var, Fréjus [ $\left.43^{\circ} 26^{\prime} \mathrm{N}, 6^{\circ} 44^{\prime} \mathrm{E}\right]$, VIII.1951 (MHNG); $10^{\circ}, 1$ 웅, Meailles [440.' $\mathrm{N}, 6^{\circ} 38^{\prime} \mathrm{E}$ ], 13.-17.IV.1949 (MHNG); $10^{\top}$, La Cayolle [ $43^{\circ} 14^{\prime} \mathrm{N}, 5^{\circ} 23^{\prime} \mathrm{E}$ ], 14.VII.1938 (MHNG);

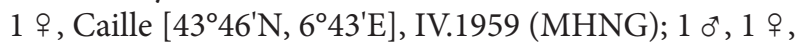

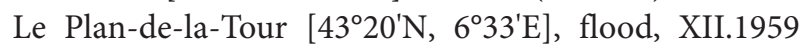

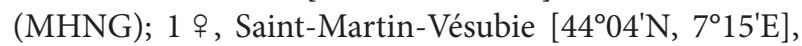
leg. Buchet (MHNG); 1 ㅇ, ex., Alpes-Maritimes, "Pont du Pali", 20.III.1946 (MHNG). 
Germany: Niedersachsen: $10^{\star}, 1$ ㅇ, Northeim env., Weper, pitfall, 4.VIII.1989, leg. Joger (cAss); $10^{*}$, same data, but 28.IV.1988 (cAss); 1 ㅇ, same data, but 5.VI.1988 (cAss); 1 ơ, 1 ㅇ, same data, but 6.VII.1987 (cAss); 1 으, same data, but 31.VIII.1989 (cAss); 7 ơ $^{\top}, 4$ 우 우 , Lüchow-Dannenberg, Schreyahn, saline habitat, 16.VI.1987, leg. Wunderle (cWun, cAss). Niedersachsen or Sachsen-Anhalt: 3 우 우, Harz (MNB). Mecklenburg-Vorpommern: $2 \sigma^{\star} o^{*}, 2$ ㅇ ㅇ, Schönberg [53 $\left.50^{\prime} \mathrm{N}, 10^{\circ} 56^{\prime} \mathrm{E}\right], 13 . \mathrm{VII} .1917$, leg. Benick (MHNG). Sachsen-Anhalt: 1 ex., Halle env., Gimritz, fallow, pitfall, 3.VIII.1994, leg. Teichmann (cFel); 1 ex., same data, but 11.V.1994 (cFel); $1 \sigma^{*}$, Halle, leg. Ruthe (MNB); $10^{\star}$, Questenberg [51 $28^{\circ} \mathrm{N}, 11^{\circ} 07^{\prime} \mathrm{E}$ ], 25.VIII.1915, leg. Petry (MNB). Hessen: $1 \sigma^{\star}$, Naumburg, 18.IV.1920, leg. Maertens (MNB); 1 ㅇ, Marburg, 1.IV.1904 (MNB); 1 우, Kühkopf [ $\left.49^{\circ} 49^{\prime} \mathrm{N}, 8^{\circ} 26^{\prime} \mathrm{E}\right]$, 29.I.1955, leg. Vogt (MHNG). Brandenburg/Berlin: 1 , Berlin, 2.IV.1990, leg. Göllner (MNB); 1 ㅇ, Berlin-Lankwitz, Pädagogische Hochschule, fallow, 16.IV.1971 (MNB); $10^{*}$, Berlin, Bahnhof Heerstraße, 6.V.1958, leg. Puthz (MNB); 1 o $^{\star}$, same data, but 19.VI.1958 (MHNG); 1 우, Berlin, leg. Ruthe (MNB); $1 \sigma^{*}$, Berlin-Lichtenrade, Richard-Tauberdamm, fallow, 14.VI.1980, leg. Korge (MNB); $10^{\star}, 1$ ㅇ, Pankow, 1909 (MNB); $10^{\star}$, Berlin env., Marienfelde (MNB); 1 오 , Schönefeld env. (MNB); 1 ㅇ, Rüdersdorf, 17.VIII.1942 (MNB); 1 , Eberswalde

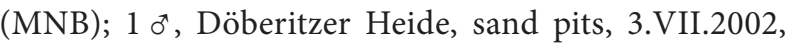
leg. Korge (MNB); 1 ๙ , Luckenwalde, IX.1903, leg. Delahon (MNB); 1 ๙ $^{*}$, same data, but V.1918 (MNB); 1 ㅇ, same data, but VII.1914 (MNB); 1 ㅇ, Schloss Güterfelde ["Gütergotz"; $52^{\circ} 22^{\prime} \mathrm{N}, 13^{\circ} 12^{\prime} \mathrm{E}$ ] (MNB); 1 우, Eisenhüttenstadt, 26.III.1982, leg. Pütz (MNB). Baden-

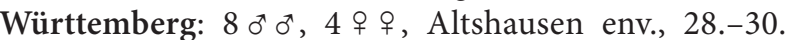
VII.1987, leg. Wunderle (cWun, cAss); $10^{\star}$, Gruibingen $\left[48^{\circ} 35^{\prime} \mathrm{N}, 9^{\circ} 38^{\prime} \mathrm{E}\right], 10 . \mathrm{V} .1964$, leg. Lohse (MHNG). Rheinland-Pfalz: $10^{\star}, 2$ 우, Bad Dürkheim, leg. Eppelsheim (NHMW); $2 \sigma^{\star} \sigma^{*}, 2$ 우 우, Speyer, XI.1882

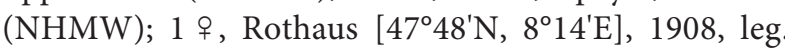
Riehn (MNB). Bayern: $10^{*}, 1$ ㅇ, München, 3.IX.1907, leg. Ihssen (MNB); $20^{\star} 0^{\star}, 7$ 우 우, München, 24.IV.1910, leg. Ihssen (MNB); 1 ㅇ, München, 9.IV.1909, leg. Ihssen (MNB); $10^{\star}$, München, 3.V.1910, leg. Ihssen (MNB); $1 \sigma^{*}$, München, 24.VIII.1911, leg. Ihssen (MNB); $10^{\star}$, Garmisch-Partenkirchen, 26.V.1942, leg. Ihssen (MNB); 2 우 우, Kastl (Oberpfalz) [49 $\left.49^{\prime} \mathrm{N}, 11^{\circ} 53^{\prime} \mathrm{E}\right]$, leg. IV.1913, leg. Waegner (MNB); $10^{*}, S$ Murnau, Eschenlohe $\left[47^{\circ} 36^{\prime} \mathrm{N}, 11^{\circ} 10^{\prime} \mathrm{E}\right]$, 3.VIII.1937, leg. Ihssen (MNB); $10^{*}, 2$ 우, Lenggries (MNB); 1 우, Lenggries-Winkl, 11.V.1964, leg. Lohse (MHNG); 1 ㅇ, Bamberg (MNB); 1 ơ, "Ob.-Bayern" leg. Arnold (MNB). Thüringen: 3 우 우, Alter Stolberg [5132' N, 1055'E], 11.XI.1917, leg. Petry (MNB); $10^{\star}$, same data, but 19.VIII.1910 (MNB); 2 o $^{\star} \sigma^{\star}, 2$ ㅇ ㅇ , Kyffhäuser, Kalktal, 26.IX.1958, leg. Dorn (MHNG, MNB). Sachsen: 2 ㅇ ㅇ, Moritzburg [5109'N, $\left.13^{\circ} 41^{\prime} \mathrm{E}\right]$, leg. Minkwitz (MNB). German or Polish territory: 2 우 우, "Pommern”, leg. Schneider (NHMW). Switzerland: 3 exs., locality not specified (MHNG).
Austria: Oberösterreich: $2 \sigma^{\star} \sigma^{\star}, 2$ ㅇ ㅇ [with workers of Formica pratensis attached to the pins], Niederranna, leg. Rupertsberger (NHMW); 1 \%, Wien, "N. Donau", leg. Meschnigg (NHMW). Tirol: $1 \mathrm{o}^{\star}$, Trins $\left[47^{\circ} 04^{\prime} \mathrm{N}\right.$, $11^{\circ} 25^{\prime} \mathrm{E}$; "F4"], leg. Franz (NHMW). Wien/Niederösterreich: $60^{\top} o^{\star}, 4$ 우 우, Wien, Bisamberg, leg. Luze (NHMW); $10^{\star}, 1$ ㅇ, Wechselgebirge, 1889, leg. Ganglbauer (NHMW); 1 온 Hollenstein [ $47^{\circ} 48^{\prime} \mathrm{N}, 14^{\circ} 46^{\prime} \mathrm{E}$ ], Krenngraben ["X 1068"], leg. Franz (NHMW); $20^{\star} 0^{*}$, 1 ㅇ, Mödling env. [“403”, “515”], leg. Franz (NHMW); 1 ㅇ, Windisch Baumgarten [48 $33^{\circ} \mathrm{N}, 16^{\circ} 43^{\prime} \mathrm{E}$ ] (MNB); 3 우 우, Lunz, Lunzberg, xerothermous slope, 10.IX.1962,

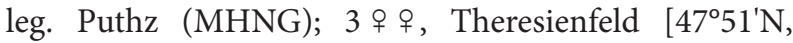
$\left.16^{\circ} 15^{\prime} \mathrm{E}\right], 20 . I V .1952$, leg. Malicky (MHNG); $10^{\star}, 1$ ㅇ, Theresienfeld, 15.III.1952, leg. Malicky (MHNG); $10^{\star}$, Kollnitz [ $\left.48^{\circ} 14^{\prime} \mathrm{N}, 15^{\circ} 08^{\prime} \mathrm{E}\right]$, 3.IV.1958 (MHNG); 2 우 우, Fischauer Vorberge $\left[47^{\circ} 49^{\prime} \mathrm{N}, 18^{\circ} 06^{\prime} \mathrm{E}\right]$, nest of mouse sifted, 16.III.1952, leg. Malicky (MHNG); 1 ㅇ, locality illegible ["Feichsbach"?], 21.VI.1961 (MHNG); 1 , locality illegible, 10.III.1961 (MHNG); 1 ㅇ, locality illegible, 1.XI.1960 (MHNG). Steiermark: 1 , , Weiz env. ["X 986”], leg. Franz (NHMW); 1 ㅇ, Baierdorf [ $47^{\circ} 10^{\prime} \mathrm{N}$, $\left.14^{\circ} 06^{\prime} \mathrm{E}\right]$ near Schöder, 7.X.1949, leg. Franz (NHMW); $40^{\top} o^{\top}, 4$ 우 우, Frohnleiten (MNB). Kärnten: 2 우 우, Maglern an der Gailitz, leg. Meschnigg (NHMW); $10^{*}$, 1 ㅇ, Villach, Seebach, leg. Meschnigg (NHMW); $20^{\star} 0^{\star}$, Heiligenblut [“G 370”], leg. Franz (NHMW); 2 우우, Mauthen [46 $\left.39^{\prime} \mathrm{N}, 1^{\circ} 59^{\prime} \mathrm{E}\right], 21 . \mathrm{VII} .1973$ (MHNG); 1 앙 Rattendorf $\left[46^{\circ} 36^{\prime} \mathrm{N}, 13^{\circ} 16^{\prime} \mathrm{E}\right], 2$.VII.1966, leg. Lohse (MHNG); $20^{\star} o^{\star}$, "Kärnten" (MNB). Burgenland: 3 우 우, Rohrbach bei Mattersburg, Marzer Kogel, nest of Formica sanguinea, 1.IV.1926, leg. Scheerpeltz (NHMW); $60^{\top} 0^{\star}, 5$ ㅇ 우, Neusiedlersee [“351”], leg. Franz (NHMW, cAss); 9 ๙ $^{\star}$, 6 우 으, Zurndorf [“345”; “74-015”], leg. Franz (NHMW, cAss); $1 o^{\star}, 2$ ㅇ 우, Illmitz, 22.VIII.1960, leg. Lohse (MHNG); 7 exs., Breitenbrunn, pitfall, VII.X.1965, leg. Malicky (MHNG); 2 exs., same data, but V-VI.1965 (MHNG); 1 ㅇ, Apetlon, pitfall, V.1967, leg. Malicky (MHNG).

Czech Republic: 3 우 우, Brandys nad Labem, leg. Skalitzky (NHMW)

Slovakia: 1 ㅇ, Plešivec $\left[48^{\circ} 32^{\prime} \mathrm{N}, 2^{\circ} 23^{\prime} \mathrm{E}\right]$ plateau, 5.V.1967, leg. Lohse (MHNG).

Italy: Trentino-Alto Adige: 1 ㅇ, Val di Non (TN), Castelfondo $\left[48^{\circ} 27^{\prime} \mathrm{N}, 11^{\circ} 06^{\prime} \mathrm{E}\right.$ ], torbiera d. Regole, $1230 \mathrm{~m}$, 2.VIII.1982, leg. Zanetti (cZan); 1 ㅇ, Bolzano (MNB); 1 ㅇ, Bressano [46 $42^{\prime} \mathrm{N}, 11^{\circ} 39^{\prime} \mathrm{E}$ ], 4.VI.1965, leg. Lohse (MHNG). Lombardia: $10^{\star}$, NE Sondrio, Montagna in Valtellina, 6.IV.1972, leg. Dioli (cZan); 1 o $^{\star}, 1$ ㅇ , Bergamo, Dalmine $\left[45^{\circ} 39^{\prime} \mathrm{N}, 9^{\circ} 36^{\prime} \mathrm{E}\right]$, Brembo river, 30.V.1979, leg. Kiener (MHNG). Veneto: $3 \sigma^{\star} \sigma^{\star}, 4$ 우 온, Venezia, San Giuliano, Ponte sul Oselin, 26.XII.1954, leg. Zecchini (cZan); 1 호 3 우 ㅇ, Venezia, San Giuliano, 21.XI.1954, leg. Zecchini (cZan, cAss). Emilia-Romagna: $10^{\star}, 1$ ㅇ, Montone, leg. Brenske (NHMW). Lazio: $10^{x}, 1$ ㅇ, Roma, Aniene river, II.1902, leg. Fiori (MHNG, cAss); 5 exs. [with worker of Formica sp. attached to the pin], Acquapendente, 14.VIII.1922, leg. Botto (MHNG). 
Hungary: 1 , Bugac National Park, grassland, pitfall trap, 30.VII.1986, leg. Galle (cAss).

Romania: 1 , Băile Herculane, 1895, leg. Ganglbauer (NHMW).

Croatia: $1 \sigma^{\top}, 1$ ex. without head and pronotum, locality not specified (NHMW); 1 ㅇ, Josipdol-Karlovac, car-net, 9.V.1990, leg. Wunderle (cWun).

Bulgaria: $1 \sigma^{\star}$, Macedonia, Sandanski-Struma, 16.VII.1986, leg. Pütz (cAss); $1 \sigma^{*}$, Sandanski-Struma, 28.IV.1985, leg. Jäger (MNB); $1 \sigma^{\star}$, Sandanski, vineyard, under stones, 5.VI.1984, leg. Pütz (cPüt); 2 ㅇ ㅇ, Sandanski, 16.-23.VII.1985, leg. Schülke (MNB); $10^{\star}, 3$ 우 우, 1 o $^{\star}$, 3 우 우, Rupite [ $\left.41^{\circ} 26^{\prime} \mathrm{N}, 23^{\circ} 15^{\prime} \mathrm{E}\right]$ near “General Todorow", 7.V.1984, leg. Hieke (MNB); 1 ○, Pimorsko, 16.IX.1977, leg. Hieke \& Uhlig (cAss).

Bosnia-Herzegovina: $3 o^{\star} o^{\star}, 2$ 우, Travnik, leg. Brandis (NHMW, cAss); 1 앙, Fatničko polje, entrance of Lepirnica cave, 17.-22.VI.2003, leg. Hlaváč (cTro); 4 ㅇ 우, Trebinje (MHNG).

Albania: $1 \sigma^{\star}, 5$ ㅇ ㅇ, Vlorë [“Avlona”], leg. v. Oertzen (MNB, NHMW, cAss).

Greece: mainland: 1 \% , Katerini, Agios Dimitrios, 800 m, 19.-21.VII.1990, leg. Schuh \& Zabransky (cAss); 1 ㅇ, Thessalia, Volos (MNB); $10^{\star}, 1$ 우, Evzoni $\left[41^{\circ} 06^{\prime} \mathrm{N}\right.$, $22^{\circ} 32^{\prime} \mathrm{E}$ ], 1.IX.1964, leg. Puthz (MHNG); 1 ㅇ, Serrai [41 $\left.05^{\prime} \mathrm{N}, 23^{\circ} 33^{\prime} \mathrm{E}\right]$, river bank, 16.VIII.1964, leg. Puthz (MHNG); 1 ㅇ "Attica” (NHMW). Evvoia: 1 ㅇ, Karystos, leg. v. Oertzen (MNB). Pelopónnisos: $40^{\top} 0^{\star}, 2$ 우 우, Killini Oros, Lake Stimfalia, 500 m, 3.IV.1992, leg. Frisch (MNB, cAss); 1 은 Killini, $37^{\circ} 55^{\prime} \mathrm{N}, 22^{\circ} 22^{\prime} \mathrm{E}, 1350 \mathrm{~m}$, car-net, 3.IV.2016, leg. Schülke (MNB); 1 o $^{*}$ Kalávrita, leg. Holtz (MNB); $1 \sigma^{\star}, 1$ ㅇ, Koumani, leg. Brenske (NHMW, cAss); $4 \sigma^{\star} o^{\star}, 4$ 우 ㅇ, Taygetos, leg. Brenske (MHNG, cAss). Corfu: $10^{\star}, 2$ 우 우, NE-Corfu, Archaravi, 20.-25.X.1991, leg. Katschak (cWun, cAss); 9 exs., locality not specified, leg. Reitter, Winkler (MHNG, NHMW, cAss). Ikaría: $2 \sigma^{*} \sigma^{x}, 1$, locality not specified, leg. v. Oertzen (MNB, cAss). Locality not specified: $1 \sigma^{*}, 1+$, locality not specified ["Graecia"], leg. Emge (NHMW); 1 o , 1 ㅇ, "Graecia" (MNB).

Ukraine: $1 \sigma^{\star}$, Transcarpathia, Vel'ke Berezne, sandy river bank, 17.V.2012, leg. Gontarenko (cAss).

Turkey: Bolu: $10 \sigma^{\star} \sigma^{\star}, 8$ 우 우 $26 \mathrm{~km} \mathrm{~S}$ Bolu, N Seben, $40^{\circ} 30^{\prime} \mathrm{N}, 31^{\circ} 36^{\prime} \mathrm{E}, 1410 \mathrm{~m}$, grassy slope with scattered pine trees, grass, moss, and shrub litter sifted, 27.III.2010, leg. Assing (cAss). Kastamonu: $1 \mathrm{o}^{*}, 5 \mathrm{~km}$ NW Kastamonu, $5 \mathrm{~km} \mathrm{NW}$ Ağlı, $41^{\circ} 44^{\prime} \mathrm{N}, 33^{\circ} 30^{\prime} \mathrm{E}$, margin of calcareous fir forest with interspersed pine trees, litter and moss sifted, 22.III.2010, leg. Assing (cAss); 1 ㅇ, same data, but floated from stream bank (cAss); 1 ㅇ, $40 \mathrm{~km} \mathrm{~N} \mathrm{Kastamonu,}$ $\mathrm{S}$ Kayneak geçidi, $41^{\circ} 42^{\prime} \mathrm{N}, 33^{\circ} 41^{\prime} \mathrm{E}, 1250 \mathrm{~m}$, calcareous slope, under stones, 10.IV.2009, leg. Assing (cAss). Sinop: 2 ㅇ ㅇ, $30 \mathrm{~km}$ NNE Boyabat, Dıranaz geçidi, exit SE tunnel, $41^{\circ} 38^{\prime} \mathrm{N}, 34^{\circ} 52^{\prime} \mathrm{E}$, calcareous grassland, under stones, 5.IV.2009, leg. Wunderle (cWun). Ordu: $10^{\top}, 1$ 우 $25 \mathrm{~km}$ S Ordu, S Kabaduz, 4049'N, 3754'E, 990 m, roadside, roots of grass and herbs, moss, 30.VII.2006, leg. Assing (cAss). Muğla: 1 i , N Fethiye, Çaliş, $36^{\circ} 40^{\prime} \mathrm{N}$, $29^{\circ} 06^{\prime} \mathrm{E}, 10 \mathrm{~m}, 1 . \mathrm{X} .2002$, leg. Assing (cAss). Aydin: $10^{\top}$, 25 km SSW Çine, Gökbel Dağ 1 , N-slope at peak, $37^{\circ} 28^{\prime} \mathrm{N}$, $28^{\circ} 00^{\prime} \mathrm{E}, 1420 \mathrm{~m}$, litter of pine and grass sifted, 6.IV.2006, leg. Assing (cAss). Konya: 1 , Beyşehir $\left[37^{\circ} 40^{\prime} \mathrm{N}\right.$, $\left.31^{\circ} 44^{\prime} \mathrm{E}\right]$, lake shore, 13.III.1979 (MHNG). Antalya: $50^{\top} \sigma^{\star}, 3$ 우 우 , ca. $70 \mathrm{~km}$ NE Fethiye, Gülübeli geçidi, east side, $36^{\circ} 50^{\prime} \mathrm{N}, 29^{\circ} 46^{\prime} \mathrm{E}, 1525 \mathrm{~m}$, north slope, litter of cushion plants, grass roots, and moss between rocks sifted, 29.III.2002, leg. Assing \& Wunderle (cAss, cWun); 1 ㅇ, same data, but grassland, under stones and sifted (cAss); $10^{\star}$, Alanya, 12.III.2000, leg. Esser (cAss); 2 ㅇ 우, Taşkesigi, 17.III.2000, leg. Esser (cAss).

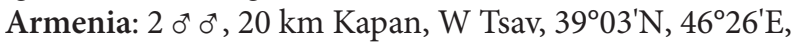
$1170 \mathrm{~m}$, stream valley, litter and flood debris near stream sifted, 9.VII.2016, leg. Assing \& Schülke (cAss, MNB).

Georgia: 1 , Kasbek (NHMW).

Russia: $10^{\star}, 1$ ㅇ, Rjasan, leg. Reitter (NHMW); 1 으, Samara, leg. Eocrsmann [?] (NHMW).

Kyrgyzstan: $1 \sigma^{*}$, Issyk-Kul, $25 \mathrm{~km}$ W Balykchy, $2 \mathrm{~km}$ S Kek Mojnok Vtoroe, 42²7'N, 75²51'E, $1670 \mathrm{~m}$, 18.VI.2011, leg. Frisch (MNB); 2 우, Tienshan, Ala Tau: Ala Artsha, 2000 m, 9.VII.1984, leg. Wrase (MNB).

Locality not specified: $3 \sigma^{\star} o^{\star}, 3$ 우 , "Styria", "Styr., etc., leg. Pipitz, etc. (MNB, NHMW); 1 ㅇ, "Silesia” (MNB);

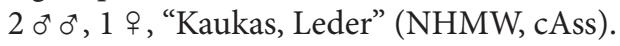

Redescription: Body length 1.7-2.5 mm; length of forebody $0.9-1.1 \mathrm{~mm}$. Coloration: body blackish, with the posterior portion of the elytra more or less extensively and more or less distinctly paler, narrowly yellowish to broadly brown; legs brown to dark-brown; antennae blackish-brown to black.

Head approximately 1.1 times as broad as long; with weakly pronounced sexual dimorphism. Eyes approximately as long as postocular region, or slightly shorter, or slightly longer (in specimens with long elytra). Antenna $0.65-0.80 \mathrm{~mm}$ long; antennomere IV weakly transverse or as long as broad; antennomeres $\mathrm{V}-\mathrm{X}$ of increasing width and increasingly transverse, $\mathrm{X}$ slightly more than 1.5 times as broad as long. Mouthparts as in Figs 1-3.

Pronotum approximately 1.1 times as broad as long and approximately as broad as head, strongly convex in crosssection; punctation subject to a more or less pronounced sexual dimorphism.

Elytra of variable length, $0.8-1.1$ times as long as pronotum; punctation dense, fine, and distinct; interstices without microsculpture and glossy. Hind wings fully developed.

Abdomen approximately as broad as elytra in specimens with short elytra and narrower than elytra in specimens with long elytra; punctation denser and coarser in anterior than in posterior portions of tergites; posterior margin of tergite VII with palisade fringe; posterior margin of tergite VIII (Figs 4-9) usually broadly and weakly, rarely deeply concave, with unmodified long marginal setae, in postero-lateral portions with dense long and thin setae. 
$\sigma^{\top}:$ head often more or less distinctly depressed in posteromedian portion, punctation fine, but distinct; pronotum with very dense and often somewhat asperate punctation, with more or less pronounced median sulcus (this sulcus more distinct posteriorly than anteriorly), and sometimes with depression or shallow median impression in posterior half; sternite VIII (Figs 10-12) strongly produced posteriorly, in the middle weakly convex (nearly truncate) and with numerous long thin setae; median lobe of aedeagus (Figs 13-30) of rather variable size, 0.23 $0.28 \mathrm{~mm}$ long, at base of ventral process usually with, rarely without, more or less pronounced membranous median projection (lateral view); ventral process apically weakly incised and laterally weakly angulate (ventral view).

†: head not depressed in postero-median portion, punctation finer and less distinct than in male; pronotum with shallower, or without, median sulcus, punctation moderately dense, moderately fine, not asperate; sternite VIII with broadly convex posterior margin; spermatheca small and shaped like a comma, not distinctive.

Intraspecific variation: This species is subject to pronounced, partly clinal variation. While in populations from West and Central Europe the elytra are shorter than the pronotum and approximately as broad as the abdomen, they tend to be longer than the pronotum and broader than the abdomen in Southeast Europe and Asia. Also, external characters such as the punctation and the modifications of the male head and pronotum may vary considerably. Moreover, in one examined male from Bulgaria, the posterior margin of the male tergite VIII is much more strongly concave than is usually the case (Fig. 9). Finally, the membranous carina at the base of the ventral process of the aedeagus may be of various shapes (Figs 13-25). In material from southern Turkey, this carina is more pronounced and more obliquely truncate than in populations from West and Central Europe and from most of the Balkans. On the other hand, it may be nearly completely reduced in material from Corfu, the Pelopónnisos, Albania, and mainland Greece. However, transitional conditions were observed for all these characters, suggesting that these differences should be interpreted as intra- rather than interspecific variation.

Distribution and natural history: Anaulacaspis nigra is the most widespread species of the genus, its confirmed distribution ranging from Northeast Spain and France across Central and South Europe, Russia, Turkey, and Armenia eastwards to Kyrgyzstan (Map 2). Plausible records are known also from Scandinavia and the Baltic states (SCHÜLKE \& SMETANA 2015). The species appears to be absent from the British Isles. Based on currently available evidence, the distribution is of the PontoMediterranean type sensu LATtin (1967). According to SchüLKe \& SMEtana (2015), the species has been recorded also from Portugal; Gamarra \& Outerelo (2005) report it from Madrid and Mallorca. However, no material from these regions was seen in the course of the present revision, and based on the currently known distribution, these records are most likely incorrect and may well refer to $A$. iberica.

The examined material was collected in various habitats such as different types of grassland (steppe, calcareous grassland, mountain slopes), fallows, sandy river banks, an inland saline habitat, a cave entrance, a vineyard, and nests of mice and of wood ants (Formica pratensis RETZIUS, 1783, F. sanguinea LATreILle, 1798). In the north of its range, A. nigra is usually confined to xerothermic habitats (sandy soils, calcareous slopes, urban fallows, etc.). Numerous specimens were sifted from grass roots, moss, and litter, some were found under stones. The altitudes range from 10 to $1670 \mathrm{~m}$. Adult specimens were found throughout the year. One specimen was collected with a car-net in May, suggesting that dispersal on the wing may take place in spring. According to HorIon (1967), A. nigra is mainly found on sandy soils and teneral adults have been observed in summer (from the end of July). LOKAy (1905), Roubal (1932), and LAPEVA-Gionova \& ILIEFF (2012) recorded it from the nests of various ant species: Formica pratensis, F. rufa Linnaeus, 1761, Lasius fulginosus (LATreille, 1798), L. niger (Linnaeus, 1758), and Tetramorium caespitum (LinNaEus, 1758).

\section{Anaulacaspis caucasica (FAgEL, 1969) \\ (Figs 31-34, Map 2)}

Melagria caucasica FAgel, 1969: $11 \mathrm{f}$.

Type material examined: Holotype $0^{\star}$ : “Caucase / nigra / nigra Hochh. / R.I.Sc.N.B. 17.479, Falagria, Coll. et det. A. Fauvel / G. Fagel det. 1969, Melagria caucasica n. sp. / Type / Anaulacaspis caucasica (Fagel), det. V. Assing 2016" (IRSNB). Paratypes: 1 o $^{\text {: }}$ "Caucasus, Araxesthal, Leder. Reitter / Falagria nigra Grav., Coll. Reitter / G. Fagel det. 1969, Melagria caucasica n. sp. / Paratype / Anaulacaspis caucasica (Fagel), det. V. Assing 2016" (IRSNB); 1 ㅇ: "Caucasus, Araxesthal, Leder Reitter / G. Fagel det. 1969, caucasica n. sp. / Paratype / Anaulacaspis caucasica (Fagel), det. V. Assing 2016” (IRSNB).

Comment: The original description is based on a male holotype from "Caucase", two male and one female paratypes from "Caucasus, Araxesthal" and one female paratype from "Caspi-M. Gebiet, Resano" (FAGEL 1969).

Additional material examined: Azerbaijan: $10^{\star}$, Xanlar ["Helenendorf”], leg. Reitter (NHMW).

Iran: Azarbayjan-e Gharbi: $9 \sigma^{\star} \sigma^{\star}, 8$ 우 우, road Khoy Siyah Chesmeh, $9 \mathrm{~km} \mathrm{~W}$ Zar Abad, $38^{\circ} 47^{\prime} \mathrm{N}, 44^{\circ} 32^{\prime} \mathrm{E}$, 1970 m, 30.VIII.2008, leg. Frisch \& Serri (MNB, cAss); $20^{\star} o^{x}, 4$ 우 우, road Khoy - Siyah Chesmeh, $17 \mathrm{~km} \mathrm{~W}$ Zar Abad, 38 $46^{\prime} \mathrm{N}, 4^{\circ} 29^{\prime} \mathrm{E}, 2640$ m, 30.VIII.2008, leg.

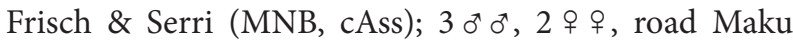
- Bazargan, $7 \mathrm{~km}$ road to Avajiq, $39^{\circ} 21^{\prime} \mathrm{N}, 44^{\circ} 19^{\prime} \mathrm{E}$, 
1520 m, 27.VIII.2008, leg. Frisch \& Serri (MNB, cAss); $100^{\star} o^{\star}, 10$ 우 우 [partly slightly teneral], road Shot - Siyah

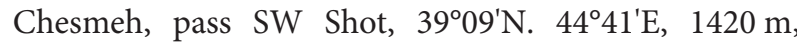
28.VIII.2008, leg. Frisch \& Serri (MNB, cAss); $10^{\star}, 1$ 우, Shot - Siyah Chesmeh road, Qarah Kelisa, 3906' N, 443'ㄹ'E, 1860 m, 28.VIII.2008, leg. Frisch \& Serri (MNB, cAss); $10^{\star}$, NW Piranshah, $7 \mathrm{~km}$ road to Hashkan, $36^{\circ} 48^{\prime} \mathrm{N}, 45^{\circ} 05^{\prime} \mathrm{E}, 1600 \mathrm{~m}, 2 . \mathrm{IX} .2008$, leg. Frisch \& Serri (cAss). Ardabil: $30^{\top} \sigma^{*}, 2$ 우 우, $15 \mathrm{~km}$ S Germi, Beldashi, $38^{\circ} 58^{\prime} \mathrm{N}, 48^{\circ} 00^{\prime} \mathrm{E}, 1270 \mathrm{~m}, 12 . \mathrm{X} .2011$, leg. Frisch (MNB, cAss).

Redescription: External characters as in A. nigra, except as follows:

Elytra often extensively brown; legs reddish to reddishbrown; antennae reddish to dark-brown.

Eyes slightly longer than postocular region in dorsal view. Elytra approximately 1.1 times as long as pronotum. Abdomen narrower than elytra; posterior margin of tergite VIII convex, in the middle broadly truncate and with moderately long marginal setae (Fig. 31).

$\sigma^{*}$ : head without depression in postero-median portion and with variable, fine and sparse to distinct and moderately dense punctation; pronotum with more or less distinct impression along middle and more or less extensively impressed in postero-median portion, punctation very dense and somewhat asperate; sternite VIII (Fig. 32) as in A. nigra; median lobe of aedeagus (Figs 33-34) approximately $0.28 \mathrm{~mm}$ long, ventral process in ventral view with a pair of conspicuous lateral projections in the middle, at base of ventral process with very weakly pronounced membranous median projection (lateral view).

은 head with very fine and sparse punctation; pronotum with weakly pronounced impression along middle, punctation moderately dense and fine, not asperate.

Distribution and natural history: Confirmed records are known only from Azerbaijan and North Iran (Map 2). The material from Iran was collected at altitudes of 1270-2640 m. Several specimens found in August are teneral.

\section{Anaulacaspis pseudonigra spec. nov.}

(Figs 35-41, Map 2)

Type material: Holotype $\sigma^{*}$ : "TR [18] - Amasya, 4 km SW Taşova, 300 m, 40 44'47"N, 36²17'41", bank of Yeşilırmak river, 17.VII.2008, V. Assing / Holotypus ơ Anaulacaspis pseudonigra sp. n., det. V. Assing 2016" (cAss). Paratypes: $1 \sigma^{*}$ : same data as holotype (cAss); 2 우 : same data, but leg. Schülke (MNB, cAss).

Etymology: The specific epithet (adjective) alludes to remarkable similarity of this species to $A$. nigra.
Description: External characters, including the modifications of the male pronotum, as in A. nigra, except as follows:

Elytra slightly longer than pronotum. Tergite VIII (Fig. 35) with convex posterior margin.

$\sigma^{*}$ : head without depression or impression in posteromedian portion; sternite VIII (Fig. 36) of similar shape and chaetotaxy as in A. nigra; median lobe of aedeagus (Figs 37-40) 0.24-0.26 mm long; ventral process longer and apically more acute than in A. nigra, at base without semi-transparent projection in lateral view, laterally with a pronounced projection on either side; crista apicalis longer.

옹 sternite VIII (Fig. 41) strongly transverse and broadly, convexly produced posteriorly.

Comparative notes: Anaulacaspis pseudonigra is best distinguished from the highly similar A. nigra by the different shapes of the abdominal tergite VIII and of the aedeagus. The similar shape of tergite VIII and particularly the similar modifications of the ventral process of the aedeagus (laterally with distinct projections in ventral view) suggest that $A$. pseudonigra is most closely allied to A. caucasica, from which it differs only by the morphology of the median lobe of the aedeagus (smaller; shape of ventral process in lateral view; lateral projections of ventral process of different shape and closer to base of ventral process).

Distribution and natural history: The type locality is situated in Amasya province, northern Anatolia (Map 2). The specimens were hand-collected from mud and sand on a river bank at an altitude of $300 \mathrm{~m}$.

\section{Anaulacaspis reticulata spec. nov. (Figs 42-46, Map 2)}

Type material: Holotype $\sigma^{\top}$ : “TR Mersin (46), Kirobasi-

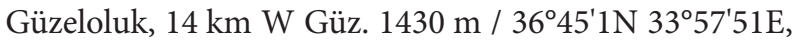
(46) leg. 8.5.2004, Brachat \& Meybohm / Holotypus $\sigma^{\star}$ Anaulacaspis reticulata sp. n., det. V. Assing 2016" (cAss).

Etymology: The specific epithet (Latin, adjective) alludes to the conspicuous microreticulation on the elytra.

Description: Body length $2.2 \mathrm{~mm}$; length of forebody $1.0 \mathrm{~mm}$. Coloration: body black; legs and antennae dark-brown.

Head 1.15 times as broad as long, with weakly pronounced sexual dimorphism; punctation fine and moderately dense; interstices without microsculpture. Eyes longer than postocular region in dorsal view. Antennae short and massive; antennomere IV distinctly transverse; antennomeres $\mathrm{V}-\mathrm{X}$ of gradually increasing width and increasingly transverse, $\mathrm{X}$ approximately twice as broad as long. 
Pronotum distinctly transverse, more than 1.15 times as broad as long and slightly broader than head; sexual dimorphism weakly pronounced; punctation dense and moderately fine; postero-median portion with microreticulation.

Elytra as long as pronotum; punctation dense and distinct; interstices with a distinct network of microstriae (Fig. 42). Hind wings fully developed.

Abdomen slightly narrower than elytra; punctation fine, rather dense, and more or less regularly distributed; interstices with microreticulation composed of larger meshes on tergites III-V and of smaller meshes on tergites VI and VII; posterior margin of tergite VIII (Fig. 43) weakly convex, postero-laterally with a cluster of dense long setae; marginal setae thin and long.

$o^{*}$ : head slightly depressed in postero-median portion; pronotum with a fine median sulcus in posterior two-thirds, in postero-median portion with denser punctation; sternite VIII (Fig. 44) with convex posterior margin, marginal setae long, thin, and distributed along all of posterior margin; median lobe of aedeagus (Figs 45-46) small, $0.21 \mathrm{~mm}$ long; ventral process broadbased in lateral view, very broad, laterally not angulate, and apically weakly incised in ventral view, at base without semi-transparent projection.

o : unknown.

Comparative notes: Among the species of the A. nigra group, A. reticulata is characterized by the short and massive antennae with a distinctly transverse antennomere IV, the presence of distinct microreticulation on the pronotum, the elytra, and the abdomen, less fine punctation of the elytra, the shapes and chaetotaxy of tergite VIII and the male sternite VIII, and by the morphology of the median lobe of the aedeagus.

Distribution: This species is currently known only from the type locality, which is situated in Mersin province, central southern Anatolia (Map 2), at an altitude of $1430 \mathrm{~m}$.

\section{Anaulacaspis libanotica (FAgEL, 1969) (Figs 47-51, Map 2)}

Melagria libanotica FAGEL, 1969: 12.

Type material examined: Paratypes: 1 : : "Liban / Coll. et det. A. Fauvel, Falagria nigra Grav., R.I.Sc.N.B. 17.479 / G. Fagel det. 1969, Melagria libanotica n. sp. / Paratype" (IRSNB); 1 o: same labels, but without locality labels (IRSNB); 1 o: "Borak / Coll. et det. A. Fauvel, Falagria nigra Grav., R.I.Sc.N.B. 17.479 / G. Fagel det. 1969, Melagria libanotica n. sp. / Paratype" (IRSNB).

Comment: The original description is based on a male holotype from "Liban : Moukhtara, 800 m", two females from "Liban", one from "Liban, Borak", and one from
"Syrien, Kaifa" (FAGEL 196 ). The holotype was not among the material made available to me from the IRSNB.

Additional material examined: Lebanon: 1 ㅇ, Beirouth (NHMW).

Israel: $10^{*}$, Tel Yosef [32 $\left.33^{\prime} \mathrm{N}, 35^{\circ} 24^{\prime} \mathrm{E}\right]$ (cAss); 1 우, Golan Heights, Mas'ala, Ya’ar Odem R., $33^{\circ} 13^{\prime} \mathrm{N}, 35^{\circ} 45^{\prime} \mathrm{E}$, 1015 m, 2.II.2007, leg. Feldmann (cFel); 1 \%, Golan Heights, Bental Reservoir, W Merom Golan, 1000 m, stony wetland near shore, 30.VI.2006, leg. Wrase (MNB). Locality not specified: 3 우, "Syria” (NHMW, cAss).

Redescription: Body length 2.3-2.6 mm; length of forebody 1.1-1.3 mm. Coloration: body blackish, with the sutural and posterior portions of the elytra usually somewhat paler brown; legs pale-brown to brown; antennae dark-brown with the basal antennomeres sometimes more or less distinctly paler.

Head approximately 1.1 times as long as broad, with fine and rather dense punctation and glossy; sexual dimorphism weakly pronounced. Eyes slightly longer than postocular region in dorsal view. Antenna $0.8-0.9 \mathrm{~mm}$ long; antennomeres IV approximately as long as broad, $\mathrm{V}-\mathrm{X}$ of gradually increasing width and increasingly tranverse, and $\mathrm{X}$ approximately 1.5 times as broad as long.

Pronotum approximately 1.1 times as broad as long and as broad as head, glossy; punctation moderately dense and extremely fine.

Elytra approximately as long as pronotum, with fine and rather dense punctation. Hind wings present.

Abdomen slightly narrower than elytra; punctation fine and rather dense; interstices with microreticulation, this microreticulation more distinct on posterior than on anterior tergites; tergite VIII (Fig. 49) with postero-lateral cluster of long thin setae on either side and with truncate to concave posterior margin, marginal setae rather long and rather stout.

$0^{*}$ : head neither impressed nor depressed, punctation slightly more distinct than in female; pronotum without distinct median impression, punctation denser and more distinct than in female; sternite VIII (Fig. 50) strongly produced posteriorly, apically with very dense and long setae; median lobe of aedeagus (Figs 47-48) $0.32 \mathrm{~mm}$ long, very slender in lateral view, broad and apically abruptly narrowed in ventral view.

+ : posterior margin of sternite VIII (Fig. 51) strongly convexly produced and with dense long and fine setae in the middle.

Comparative notes: As can be inferred from the chaetotaxy of tergite VIII (with postero-lateral cluster of long thin setae) and from the shape of the male and female sternites VIII, A. libanotica belongs to the A. nigra group. It is distinguished from other similarly black and finely punctate species of this group by the near absence of a sexual dimorphism of the head and pronotum, as well as by the morphology of the longer median lobe of the aedeagus. 
Distribution and natural history: Confirmed male-based records are currently known only from Lebanon and Israel (Map 2). The specimens from Israel were collected at an altitude of approximately $1000 \mathrm{~m}$, some of them in a stony wetland near the shore of a reservoir.

\section{Anaulacaspis sinuata spec. nov.} (Figs 52-56, Map 2)

Type material: Holotype ơ : "IRAN KORDESTÂN, E de Marivân, 35³2'N, 46² $20^{\prime} \mathrm{E}$, A. Senglet 16.9.75 / Holotypus o Anaulacaspis sinuata sp. n., det. V. Assing 2016" (cAss). Paratypes: 19 : same data as holotype (cAss); $10^{7}$, 2 ㅇ 우 : "IRAN, Kordestan Province, Sanandaj - Divandarreh road, $21 \mathrm{~km} \mathrm{~S} \mathrm{Divandarreh,} 1890 \mathrm{~m}, \mathrm{~N} 35^{\circ} 45^{\prime} 43^{\prime \prime} \mathrm{E}$ 04704'43", 06.09.2008, lg. Frisch \& Serri” (MNB, cAss); $2 \sigma^{*} \sigma^{*}$ : "IRAN, Lorestan Province, $20 \mathrm{~km}$ SW Borujerd, 1740 m, N 3346'23" E 048³9'06", 15.10.2011, leg. Frisch” (MNB, cAss).

Etymology: The specific epithet (Latin, adjective) alludes to the sinuate ventral process of the aedeagus (lateral view).

Description: Body length 2.0-2.3 mm; length of forebody 1.0-1.1 mm. Coloration: body black, sometimes with the postero-sutural portion of the elytra indistinctly and diffusely paler; legs blackish-brown; antennae black. Integument very glossy.
Head weakly transverse, $1.05-1.10$ times as broad as long, without appreciable sexual dimorphism; punctation extremely fine and moderately dense; interstices without microsculpture. Eyes slightly longer than postocular region in dorsal view. Antennae approximately $0.8 \mathrm{~mm}$ long; antennomere IV approximately as long as broad; antennomeres $\mathrm{V}-\mathrm{X}$ of gradually increasing width and increasingly transverse, $\mathrm{X}$ approximately 1.5 times as broad as long.

Pronotum 1.10-1.15 times as broad as long and approximately as broad as head; sexual dimorphism indistinct; punctation moderately dense and fine, usually slightly more distinct than that of head.

Elytra slightly longer than pronotum; punctation moderately dense and very fine. Hind wings fully developed.

Abdomen slightly narrower than elytra; punctation fine and distinct, denser in anterior than in posterior portions of tergites; tergite VIII (Fig. 52) with postero-lateral cluster of long thin setae on either side, posterior margin truncate or indistinctly convex, with thin marginal setae.

$\sigma^{*}$ : pronotum usually with very fine and shallow median sulcus; sternite VIII (Fig. 53) strongly, convexly produced posteriorly; median lobe of aedeagus (Figs 54-55) small, 0.21-0.23 mm long; ventral process sinuate in lateral view, broad and apically not incised in ventral view.

\%: pronotum with or without very indistinct median sulcus; sternite VIII (Fig. 56) with distinctly convex posterior margin.

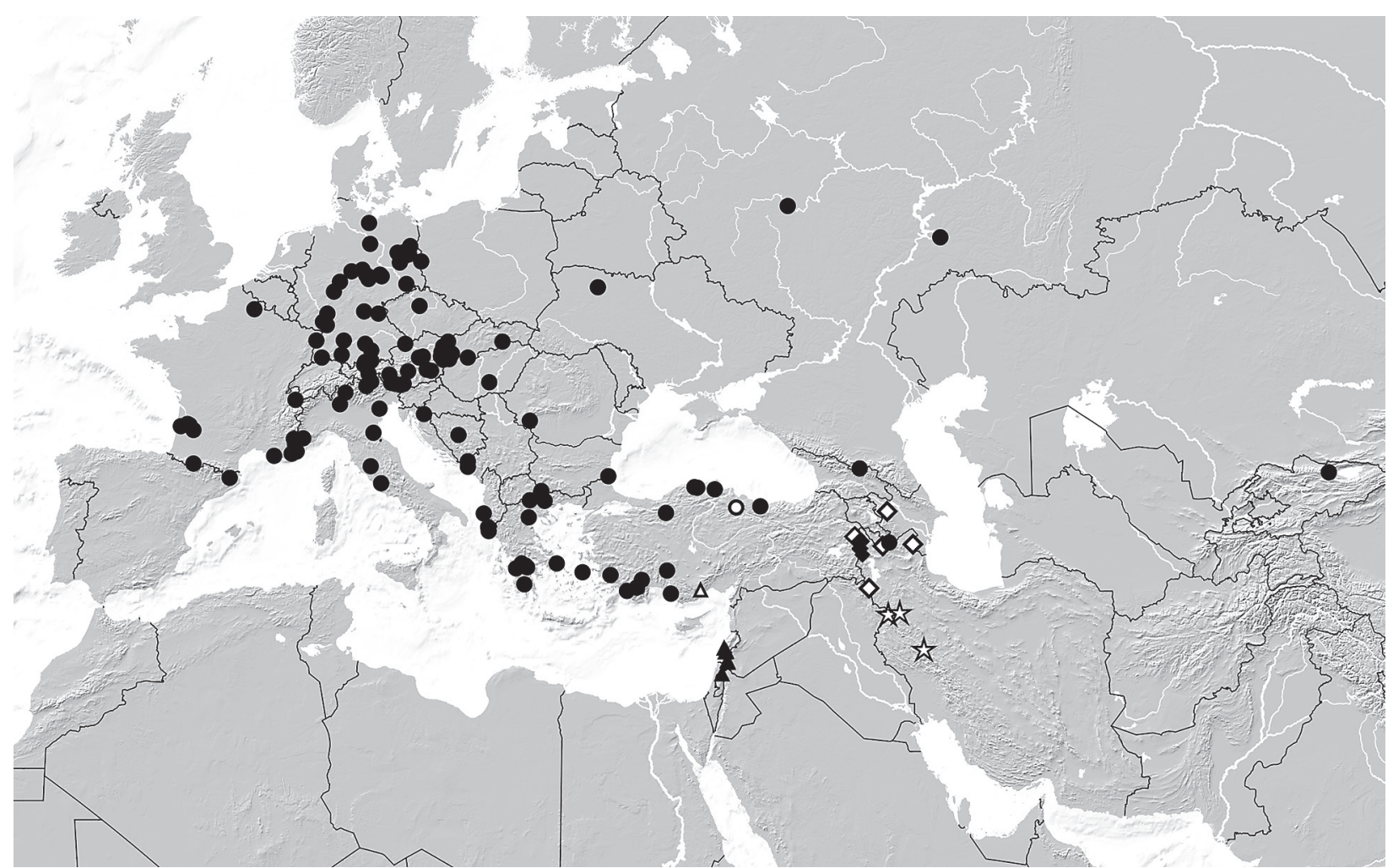

Map 2: Distributions of Anaulacaspis nigra (black circles), A. pseudonigra (white circle), A. reticulata (white triangle), A. libanotica (black triangles), A. caucasica (white diamonds), A. truncata (black diamonds), and A. sinuata (stars) in the West Palaearctic and Middle Asia, based on revised records. 
Comparative notes: Anaulacaspis sinuata is reliably distinguished from other dark-coloured species of the A. nigra group only by the shape of the median lobe of the aedeagus. In external appearance (blackish-brown legs; glossy and finely punctured forebody, it is similar to A. nigrina, from which it additionally differs by less dense punctation of the head and pronotum, usually uniformly black elytra, a truncate posterior margin of the abdominal tergite VIII, and a posteriorly more strongly produced male sternite VIII. In coloration, glossy appearance, fine punctation, the absence of a distinct sexual dimorphism of the head and pronotum, and the lateral aspect of the ventral process of the aedeagus, $A$. sinuata also resembles A. nigerrima, from which it is distinguished by the shape of tergite VIII, and the distinctly smaller median lobe of the aedeagus with a more strongly curved (lateral view), apically less acute (lateral view), smoothly converging lateral margins (ventral view), and apically not distinctly pointed ventral process (ventral view).

Distribution: The currently known distribution is confined to few localities in Kordestan and Lorestan provinces, Iran (Map 2). The altitudes indicated on the labels range from 1740 to $1890 \mathrm{~m}$.

\section{Anaulacaspis nigerrima (BernHAUER, 1908)}

(Figs 57-61, Map 3)

Falagria nigerrima Bernhauer, 1908: $38 \mathrm{f}$.

Type material examined: Lectotype $\sigma^{\star}$, present designation [dissected prior to present study]: “ $\sigma^{\star} /$ Buchara / nigerrima Bh. Cotypus / Chicago NHMus, M. Bernhauer Collection / Syntypus Falagria nigerrima Bernhauer,
V.I. Gusarov rev. 2015 / Anaulacaspis nigerrima(Bh), V.I. Gusarov det. 2015 / Lectotypus o Falagria nigerrima Bernhauer, desig. V. Assing 2016 / Anaulacaspis nigerrima (Bernhauer), det. V. Assing 2016" (FMNH). Paralectotypes: $1 \sigma^{*}$ : “ $\sigma^{*} / 124$. / Buchara / laevigata Epp., Knauth / nigerrima Bh. / nigerrima Brh. Typus" (FMNH); $1 \sigma^{\star}:$ “ $\sigma^{\star} /$ Margelan / nigerrima Bh. Cotypus" (FMNH); $1 \sigma^{*}$ : “o / Sibiria or., Reitter / nigerrima Bh., det. Bernh." (FMNH); 2 ㅇ : "Turcmenien. Reitter. Leder. / nigerrima Brh. Cotypus" (FMNH). All paralectotypes dissected prior to present study and additionally with the following labels: "Chicago NHMus, M.Bernhauer Collection / Syntypus Falagria nigerrima Bernhauer, V.I. Gusarov rev. 2015 / Anaulacaspis nigerrima (Bh), V.I. Gusarov det. 2015 / Paralectotypus Falagria nigerrima Bernhauer, desig. V. Assing 2016 / Anaulacaspis nigerrima (Bernhauer), det. V. Assing 2016".

Comment: The original description is based on an unspecified number of syntypes from "Buchara, Margelan, Turkmenien, Turkestan (Mts. Ghissar) und Ostsibirien" (BERNHAUER 1908). Six syntypes, four males and two females are deposited in the Bernhauer collection at the FMNH. One of the males from "Buchara" is designated as the lectotype.

Additional material examined: Iran: Razavi Khorasan: $40^{\star} 0^{\top}, 2$ ㅇ ㅇ, 20 km NW Torbat-e, Heydariyeh, Senobar, $35^{\circ} 26^{\prime} \mathrm{N}, 59^{\circ} 06^{\prime} \mathrm{E}, 1730 \mathrm{~m}, 28 . V .2006$, leg. Frisch \& Serri (MNB, cAss).

Afghanistan: 1 오 N Herat, SW Khouchk, Tchaouni Khoch Robat, 29.VI.1959, leg. Lindberg (NHMW).

Turkmenistan: 10 exs., locality not specified ["Turcmenien”, “Turkestan”], leg. Reitter (MHNG, MNB, NHMW, cAss).

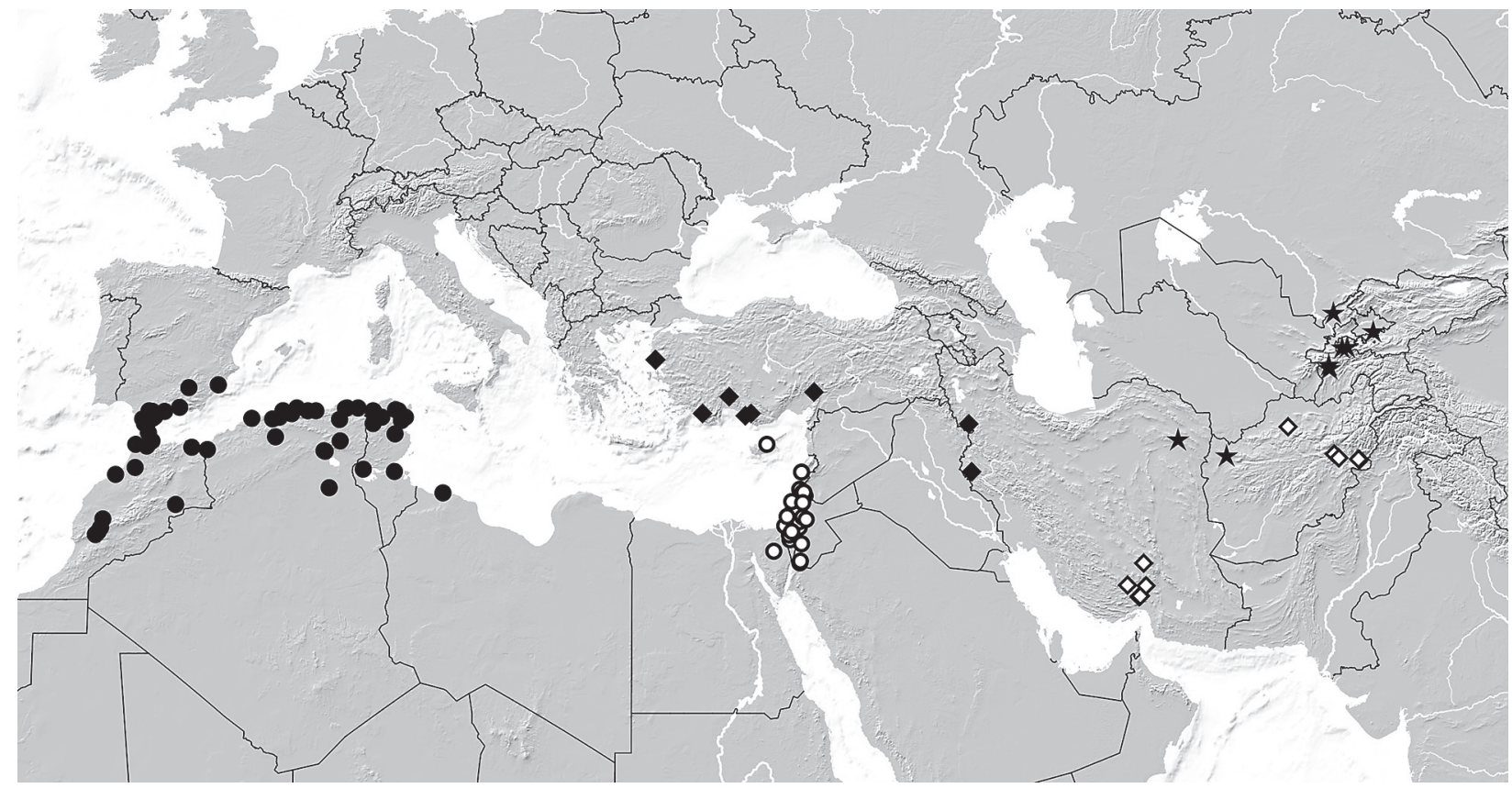

Map 3: Distributions of Anaulacaspis formosa (black circles), A. elegans (white circles), A. nigrina (black diamonds), A. eminens (white diamonds), and A. nigerrima (stars) in the West Palaearctic and Middle Asia, based on revised records. 
Kyrgyzstan: $20^{\star} 0^{\star}, 2$ 우 우, Batken, S Ay-Kol, Teo-Jailoo Valley, Tegir-Malik River, $39^{\circ} 43^{\prime} \mathrm{N}, 69^{\circ} 42^{\prime} \mathrm{E}, 2100 \mathrm{~m}$, 28.VI.2012, leg. Frisch (MNB, cAss); 2 ๙ $^{\star}, 1$ ㅇ, Batken, Isfana - Isfara, WSW Ozgorush, Turkestan range, Lyailek River, $39^{\circ} 44^{\prime} \mathrm{N}, 70^{\circ} 00^{\prime} \mathrm{E}, 1520 \mathrm{~m}, 22 . \mathrm{VI} .2012$, leg. Frisch (MNB, cAss); $20^{\top} o^{\star}, 1$ ㅇ, Batken, Isfana - Isfara, WSW Ozgorush, tributary of Lyailek River, $39^{\circ} 44^{\prime} \mathrm{N}, 70^{\circ} 00^{\prime} \mathrm{E}$, 1560 m, 23.VI.2012, leg. Frisch (MNB); 1 ex., Ferghansky Alatau, Toskool-Ata, 1.VIII.2002 (MNB); 1 ex., Kyrghyzky Alatau, foothills, O.to-Say, 1000 m (MNB).

Uzbekistan: 11 exs., Taškent, leg. Leder, Reiter, Faust, Staudinger (MNB, NHMW, cAss); 3 exs., Buchara (MHNG, MNB).

Tajikistan: $1 \mathrm{o}^{\top}$, Varzob district, Varzob, Campus 'Chaika', $38^{\circ} 46^{\prime} \mathrm{N}, 68^{\circ} 49^{\prime} \mathrm{E}, 1110 \mathrm{~m}, 7 .-8 . V I I .2012$, leg. Barševskis (cAss); 1 ex., Varzob district, 48 km N Dushanbe, Khodja Obi Gharm, 1900 m, 3.-4.VII.2012, leg. Barševskis (cAss); 37 exs., Hissar-Alai, Adjuk cleft near Varzob, 1200 m, stream bank, 1.-3.VII.1990, leg. Schülke, Wrase (MNB, cAss, cWun).

Country not specified: 6 exs., “Transkaspien”, leg. Reitter (NHMW).

Redescription: External characters as in A. nigra, except as follows:

Whole body black including antennae black, legs blackish brown (old material usually paler, with the pronotum and elytra brown and the legs pale-brown).

Head and pronotum with very fine and rather sparse punctation, without distinct sexual dimorphism. Eyes of somewhat variable length, $0.9-1.1$ times as long as pronotum. Posterior margin of tergite VIII broadly concave (Fig. 57).

$\mathrm{o}^{\star}$ : sternite VIII (Fig. 58) obtusely pointed posteriorly; median lobe of aedeagus (Figs 59-61) 0.27-0.30 mm long, ventral process apically acute (both in ventral and in lateral view), distinctly sinuate, and without membranous median projection at base (lateral view).

Distribution and natural history: Anaulacaspis nigerrima is widespread and apparently not uncommon in Middle Asia (Northeast Iran, Turkmenistan, Kyrgyzstan, Uzbekistan, Tajikistan, Afghanistan) (Map 3). One paralectotype was collected in an unspecified locality in Siberia ("Siberia or."). The altitudes indicated on the labels range from 1000 to $2100 \mathrm{~m}$. At least some of the specimens were collected on the banks of rivers and streams.

\section{Anaulacaspis persica (FAGEL, 1969)}

(Figs 62-69, Map 5)

Melagria persica FAGEL, 1969: $37 \mathrm{f}$.

Type material examined: Holotype $0^{\star}:$ "Persia settent., 1862-63. Coll. G. Doria / Coll. et det. A. Fauvel, Falagria naevula Er., R.I.Sc.N.B. 17.479 / G. Fagel det. 1968, Falagria persica n. sp. / Type / Anaulacaspis persica
(Fagel), det. V. Assing 2016" (IRSNB). Paratypes: $10^{\text {t }}$ : same data as holotype (IRSNB); $1 \mathrm{o}^{\text {: }}$ "Persia merid., 1862-63. Coll. G. Doria / Coll. et det. A. Fauvel, Falagria naevula Er., R.I.Sc.N.B. 17.479 / G. Fagel det. 1968, Falagria persica n. sp. / Paratype / Anaulacaspis persica (Fagel), det. V. Assing 2016” (IRSNB).

Comment: The original description of Melagria persica is based on a male holotype and one paratype from "Persia settent." and one paratype from "Persia merid." (FAGEL 1969). Aside from the three type specimens listed above, there is an additional specimen labelled as paratype of Melagria persica by Fagel in the Fauvel collection (locality label illegible). Since it is not mentioned in the original description, it does not have type status.

Additional material examined: Iran: AzarbayjanGharbi: $20^{\star} o^{\star}, 2$ 우 우 [partly slightly teneral], Shot - Siyah Chesmeh road, pass SW Shot, $39^{\circ} 08^{\prime} \mathrm{N}, 44^{\circ} 38^{\prime} \mathrm{E}, 1760 \mathrm{~m}$, 28.VIII.2008, leg. Frisch \& Serri (MNB, cAss); $10^{\star}$, Shot - Siyah Chesmeh road, Qarah Kelisa, 390' $\mathrm{N}, 44^{\circ} 33^{\prime} \mathrm{E}$, 1860 m, 28.VIII.2008, leg. Frisch \& Serri (MNB); $10^{\star}$, 3 우우, road Maku - Bazargan, $7 \mathrm{~km}$ road to Avajiq, $39^{\circ} 21^{\prime} \mathrm{N}, 44^{\circ} 19^{\prime} \mathrm{E}, 1520 \mathrm{~m}, 27 . \mathrm{VIII} .2008$, leg. Frisch \& Serri (MNB, cAss). Razavi Khorasan: $10^{*}, 2$ exs., 27 km SW Chanaran, SW Frizi, 36²8'N, 58 57'E, 1690 m, 29.V.2006, leg. Frisch \& Serri (MNB, cAss). Ilam: $10^{\star}, 2$ exs., $10 \mathrm{~km}$ NW Eyvan, $5 \mathrm{~km}$ W Alamdar, $33^{\circ} 52^{\prime} \mathrm{N}, 46^{\circ} 11^{\prime} \mathrm{E}, 1170 \mathrm{~m}$, 18.X.2011, leg. Frisch (MNB, cAss). Chahar Mahal-oBakhtiyari: $10^{\star}$, Izeh-Lordigan, 7 km SW Manj, 31 $34^{\prime} \mathrm{N}$, 50³4'E, 1250 m, 20.X.2011, leg. Frisch (cAss). Kerman: $10^{\star}, 1$ ex., 6 km SE Saghdar, Banestan, 28 $50^{\prime} \mathrm{N}, 57^{\circ} 55^{\prime} \mathrm{E}$, 1930 m, 27.V.2010, leg. Frisch (MNB, cAss).

Kyrgyzstan: 2 우, Turkestan range, Layle Mazar valley, Katran env., 1700 m, 23.VI.2003, leg. Lackner (cAss); $6 \mathrm{o}^{\top} \mathrm{o}^{\star}$, Batken, S Quvasoy, Majdan, $40^{\circ} 05^{\prime} \mathrm{N}, 72^{\circ} 04^{\prime} \mathrm{E}$, 1370 m, 17.VI.2012, leg. Frisch (MNB, cAss).

Redescription: Body length 2.1-2.5 mm; length of forebody 1.1-1.2 mm. Coloration: head reddish to dark-brown; pronotum reddish; elytra yellowish to reddish-yellow with a more or less pronounced and more or less extensive medio-lateral dark spot; abdomen darkbrown to blackish-brown with the posterior portions of segments VII and VIII and usually also the posterior and lateral portions of segments III-V, rarely all of segments III-V paler; legs pale-yellowish; antennae yellowishbrown, with the basal 3-4 antennomeres yellow.

Head distinctly transverse, $1.20-1.25$ times as broad as long, with weakly pronounced sexual dimorphism; punctation moderately sparse. Eyes distinctly longer than postocular region in dorsal view. Antenna $0.9-1.0 \mathrm{~mm}$ long and rather slender; antennomere IV approximately as long as broad; antennomeres $\mathrm{V}-\mathrm{X}$ increasingly transverse and of gradually increasing width, $\mathrm{X}$ approximately 1.5 times as broad as long.

Pronotum indistinctly transverse, $1.05-1.10$ times as broad as long and approximately as broad as, or slightly 
narrower than, head; sexual dimorphism weakly pronounced.

Elytra as long as, or slightly longer than, elytra; punctation moderately dense and very fine. Hind wings fully developed.

Abdomen narrower than elytra; punctation of tergites denser and more distinct in anterior than in posterior portions of tergites; posterior margin of tergite VIII (Figs 62-63) conspicuously produced in the middle, this projection with a fringe of dense long and thin setae, postero-laterally with a pronounced cluster of long thin setae on either side.

$\sigma^{*}$ : head with indistinct postero-median depression or impression and with moderately fine punctation; pronotum with fine median sulcus and relatively indistinct impression in postero-median portion, punctation similar to that of head; sternite VIII (Fig. 64) with strongly convex or obtusely pointed posterior margin; median lobe of aedeagus (Figs 65-69) approximately $0.25 \mathrm{~mm}$ long; ventral process apically of broadly triangular shape in ventral view.

o: head with very fine punctation; pronotum without median sulcus and with very fine punctation; sternite VIII with broadly convex posterior margin.

Intraspecific variation: The postero-median process of the abdominal tergite VIII is usually of more or less triangular shape. In one male from Iran, however, this process is apically rounded.

Comparative notes: Anaulacaspis persica is distinguished from its congeners of similarly small size and coloration particularly by the conspicuous shape of tergite VIII and by the shape of the median lobe of the aedeagus.

Distribution and natural history: This species has been recorded from several localities in Iran and in Kyrgyzstan (Map 5). The altitudes indicated on the labels range from 1170 to $1930 \mathrm{~m}$. Teneral specimens were found in August.

\section{Anaulacaspis truncata spec. nov.}

(Figs 70-75, Map 2)

Type material: Holotype ơ: "IRAN, Azarbayjan-e Gharbi, Khoy - Qotur road: $32 \mathrm{~km} \mathrm{~W} \mathrm{Khoy} \mathrm{(Qotur} \mathrm{River),}$ 1540 m, N 38²7'33" E 044³9'19", 29.08.2008, lg. Frisch \& Serri / Holotypus $0^{\star}$ Anaulacaspis truncata sp. n., det. V. Assing 2016" (MNB). Paratypes: $4 o^{\star} o^{\star}, 3$ ㅇ 우 : same data as holotype (MNB, cAss); 4 ㅇ ㅇ: "IRAN, Azarbayjan-e Gharbi, Khoy - Siyah Chesmeh road: $21 \mathrm{~km}$ W Zar Abad, 2350 m, N 3844'19" E 044²8'09", 30.08.2008, lg. Frisch \& Serri” (MNB, cAss).

Etymology: The specific epithet (Latin, adjective) alludes to the truncate postero-median process of the abdominal tergite VIII.
Description: Body length 1.9-2.5 mm; length of forebody 1.0-1.2 mm. Coloration: head blackish-brown; pronotum reddish-brown to brown; elytra with an extensive medio-lateral infuscate spot, humeral angles and anterior portion of sutural region reddish-yellow to reddish, posterior margin and posterior portion of sutural region yellowish to dark-yellowish; abdomen blackish; legs yellowish; antennae brown, with the basal 3-4 antennomeres reddish to reddish-brown.

Head 1.15-1.20 times as broad as long, without appreciable sexual dimorphism; punctation fine and moderately dense; interstices without microsculpture. Eyes longer than postocular region in dorsal view. Antennae approximately $0.9 \mathrm{~mm}$ long; antennomere IV approximately as long as broad; antennomeres $\mathrm{V}-\mathrm{X}$ of gradually increasing width and increasingly transverse, $\mathrm{X}$ approximately 1.5 times as broad as long.

Pronotum 1.05-1.15 times as broad as long and 0.9-1.0 times as broad as head; sexual dimorphism indistinct or absent; in postero-median portion usually weakly impressed and with shallow median sulcus; punctation dense and moderately fine, denser and more distinct than that of head.

Elytra as long as, or slightly longer than, pronotum; punctation dense and fine. Hind wings fully developed.

Abdomen narrower than elytra; punctation distinct, finer on posterior than on anterior tergites and denser in anterior portions than in posterior portions of tergites; posterior tergites sometimes with distinct microreticulation; tergite VIII with weakly pronounced sexual dimorphism, postero-laterally with a cluster of long setae on either side, posterior margin with a conspicuous truncate median process, this process apically with a fringe of dense long setae.

$\sigma^{*}$ : punctation of pronotum on average slightly more distinct than in female; posterior process of tergite VIII more pronounced (Fig. 70); posterior margin of sternite VIII (Fig. 71) produced and obtusely angled in the middle, marginal setae long, thin, and distributed across all of posterior margin; median lobe of aedeagus (Figs 72-73) 0.22-0.24 mm long; ventral process strongly curved and narrow in lateral view, basally broad and apically weakly incised in ventral view.

o : posterior process of tergite VIII less pronounced than in male (Fig. 74); sternite VIII (Fig. 75) with broadly and strongly convex posterior margin.

Intraspecific variation: One of the females from the type locality has the posterior margin of the abdominal tergite VIII smoothly convex. Since no additional characters suggesting that it should represent a different species, this character is interpreted as a teratology.

Comparative notes: As can be inferred from the synapomorphic modifications of the abdominal tergite VIII (posteriorly with a conspicuous median process) and from the similar morphology of the aedeagus, A. truncata is closely allied to A. persica, from which it differs 
by denser and more distinct punctation of the pronotum, the shape and chaetotaxy of the abdominal tergite VIII, and by the shape of the ventral process of the aedeagus both in lateral and in ventral view.

Distribution: The known distribution is confined to two localities (altitudes: 1540 and $2350 \mathrm{~m}$ ) in Azarbayjan-e Gharbi province, Iran (Map 2).

\section{Anaulacaspis convexa spec. nov. (Figs 76-83, Map 6)}

Type material: Holotype ơ: "TURKEY 21.-24.VII.2000, Adana prov., Feke env., I. Smatana leg. / Holotypus $\sigma^{\star}$ Anaulacaspis convexa sp. n., det. V. Assing 2016” (MNB). Paratypes: $4 \sigma^{\top} \sigma^{\star}, 4$ 우 우: "Anatolia centr., Korge \& Heinz leg. / Flußufer nördl. Tunqeli, 26.VII.65” (MNB, cAss); 1 $9:$ "TR [21] - Amasya, $22 \mathrm{~km}$ NE Amasya, WNW Aktaş, 4048'30"N, 3559'30"E, 1200 m, 18.VII.2008, V. Assing" (cAss); 1 đo: "S-Türkei: Region Antalya, 2 km NW Arif, 016) feinschotterige Tropfwasserstelle unter Brücke, 20.III.2001, leg. Rose / Koordinaten (WGS 84): N $36^{\circ} 31^{\prime} 23,3^{\prime \prime}$, E $30^{\circ} 00^{\prime} 42,8,795$ m” (MNB).

Etymology: The specific epithet (Latin, adjective) alludes to the convex postero-median process of the abdominal tergite VIII.

Description: Body length 2.2-2.5 mm; length of forebody 1.1-1.2 mm. Coloration: head blackish-brown to black; pronotum reddish to brown; elytra with an extensive, but weakly defined medio-lateral infuscate spot, humeral angles and anterior portion of sutural region reddishyellow to reddish, posterior margin and posterior portion of sutural region yellowish to dark-yellowish; abdomen blackish with segments III-IV and VIII-X brown to dark-brown; legs yellowish; antennae brown, with the basal 3-4 antennomeres reddish to reddish-brown.

Head approximately 1.1 times as broad as long, with weakly pronounced sexual dimorphism; interstices without microsculpture. Eyes distinctly longer than postocular region in dorsal view. Antennae approximately $0.9 \mathrm{~mm}$ long; antennomere IV approximately as long as broad or weakly transverse; antennomeres $\mathrm{V}-\mathrm{X}$ of gradually increasing width and increasingly transverse, $\mathrm{X}$ nearly twice as broad as long.

Pronotum 1.10-1.15 times as broad as long and 0.951.00 times as broad as head; sexual dimorphism absent; punctation moderately dense and moderately fine, more distinct than that of head.

Elytra slightly longer than pronotum; punctation dense and fine. Hind wings fully developed.

Abdomen narrower than elytra; punctation distinct, finer on posterior than on anterior tergites; posterior tergites with shallow microreticulation; tergite VIII with weakly pronounced sexual dimorphism, postero-laterally with a cluster of long thin setae on either side, posterior margin with a conspicuous convex median process, this process apically with a fringe of dense long setae.

$\sigma^{*}$ : punctation of pronotum distinct; posterior process of tergite VIII (Fig. 76) more pronounced than in female; posterior margin of sternite VIII (Fig. 77) strongly, convexly produced in the middle, marginal setae long, thin, and distributed across all of posterior margin; median lobe of aedeagus (Figs 78-81) approximately $0.24 \mathrm{~mm}$ long; ventral process strongly curved and narrow in lateral view, very broad and apically incised in ventral view.

o : head with extremely fine punctation; posterior process of tergite VIII (Fig. 82) less pronounced than in male; sternite VIII (Fig. 83) with broadly convex posterior margin.

Comparative notes: As can be inferred from the similarly modified shape and chaetotaxy of the abdominal tergite VIII and from the similar morphology of the median lobe of the aedeagus, $A$. convexa is closely allied to A. persica, A. truncata, and A. eminens. Regarding the shapes of the median lobe of the aedeagus and of the abdominal tergite VIII, it is most similar to A. truncata, from which it differs by the less pronounced and convex posterior process of the abdominal tergite VIII and by the much broader ventral process of the aedeagus (ventral view).

Distribution and natural history: This species is currently known from Adana, Antalya, Amasya, and Tunceli provinces, Turkey (Map 6). The specimens from Amasya and Antalya were floated and hand-collected, respectively, from gravel of river banks at altitudes of 1200 and approximately $800 \mathrm{~m}$, respectively.

\section{Anaulacaspis eminens spec. nov. \\ (Figs 84-90, Map 3)}

Type material: Holotype $\sigma^{\star}$ : "IRAN, Hormozgan Province, Tall-e Gerdu: Homag road, $1050 \mathrm{~m}$ (Bakhun Mts), N 2751'21" E 056²8'03", 19.04.2006, lg. Frisch \& Serri / Holotypus $0^{*}$ Anaulacaspis eminens sp. n., det. V. Assing 2016" (MNB). Paratypes: 1 ex.: same data as holotype (MNB); 34 exs.: "IRAN, Hormozgan Province, Tall-e Gerdu, 820 m (Bakhun Mts), N 27²48'45" E 056 25'26", 19.04.2006, lg. Frisch \& Serri” (MNB, cAss); 3 exs.: "IRAN, Hormozgan Province, $6 \mathrm{~km}$ NE Tall-e Gerdu, 880 m (Bakhun Mts), N 2750'05" E 056²8'24", 19.04.2006, lg. Frisch \& Serri" (MNB, cAss); 1 o $^{\star}$ : "IRAN, Hormozgan Province, W Hajiabad: Dar Agah, 1110 m, N 28²1'37" E 05542'37", 22.04.2006, lg. Frisch \& Serri" (cAss); 2 ㅇ ㅇ: "IRAN, Kerman Province, $100 \mathrm{~km}$ E Hajiabad: $4 \mathrm{~km}$ W Sorkhan, $1430 \mathrm{~m}$, N 28¹9'44" E 056 50'35", 20.04.2006, lg. Frisch \& Serri” (MNB, cAss); 1 \%: "IRAN, Kerman Province, Bardsir-Baft: $10 \mathrm{~km}$ SE Qal-eh Askar (Mt Lalehzar), 3360 m, N 29²6'01" E 056 $44^{\prime} 31^{\prime \prime}, 22.05 .2010$, lg. Frisch \& Serri" (MNB); $10^{\top}$ : 


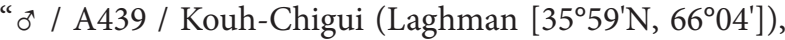
30.1.58, Steinhang / Voyage en Afghanistan, K. Lindberg / dilaticornis Scheerp. / ex coll. Scheerpeltz" (NHMW); 3 o $^{\star}$, 1 i : “A431 / Kankarak (Sorkhab [according to Internet sources close to Jalalabad, $\left.34^{\circ} 26^{\prime} \mathrm{N}, 70^{\circ} 26^{\prime} \mathrm{E}\right]$ ), 10.1.58, Steinhang, unt. Stein / Voyage en Afghanistan, K. Lindberg / dilaticornis Scheerp. / ex coll. Scheerpeltz" (NHMW); 1 ex.: "A.231. / Darountah (Djelalabad [=Jalalabad]), Flußufer, 4.1.58 et 24.1.58 / Voyage en Afghanistan, K. Lindberg / dilaticornis Scheerp. / ex coll. Scheerpeltz" (cAss); 1 \%: "Grotte de Chapar (Kabul), 23.7.57 / Voyage en Afghanistan, K. Lindberg / dilaticornis Scheerp. / ex coll. Scheerpeltz" (NHMW); $10^{*}$ : "A176 / Voyage en Afghanistan, K. Lindberg / dilaticornis Scheerp. / ex coll. Scheerpeltz" (NHMW); 1 이 “ + / Umg. Kabul, Afghanistan / Prof. Dr. R. Grill leg. et ded. / Falagria lutzi Reitter / ex coll. Scheerpeltz" (NHMW).

Etymology: The specific epithet is the present participle of the Latin verb eminere (to stand out) and alludes to the prominent crista apicalis of the aedeagus.

Description: Body length 2.3-2.6 mm; length of forebody 1.1-1.2 mm. Coloration: head brown to darkbrown; pronotum pale-reddish to dark-reddish; elytra yellowish with a relatively small medio-lateral spot; abdomen distinctly bicoloured, with segments III-IV and the anterior margin of segment $\mathrm{V}$ reddish and the remainder blackish-brown to black; legs yellowish; antennae brown with the basal 3-4 antennomeres reddish.

Head approximately 1.15 times as broad as long, without appreciable sexual dimorphism; punctation moderately dense and fine. Eyes much longer than postocular region in dorsal view. Antennae approximately $0.8 \mathrm{~mm}$ long; antennomere IV approximately as long as broad or weakly transverse; antennomeres $\mathrm{V}-\mathrm{X}$ of gradually increasing width and increasingly transverse, $\mathrm{X}$ approximately 1.5 times as broad as long.

Pronotum approximately 1.15 times as broad as long and approximately as broad as head, with or without very indistinct sexual dimorphism.

Elytra approximately as long as, or slightly longer than, pronotum; punctation dense and very fine. Hind wings fully developed.

Abdomen slightly narrower than elytra; punctation distinct, denser in anterior than in posterior portions of tergites; posterior margin of tergite VIII (Figs 84, 89) strongly convex in the middle, postero-laterally with a cluster of long thin setae; marginal setae thin and moderately long.

$0^{*}$ : pronotum on average with more distinct punctation than in female, sometimes weakly impressed in posteromedian portion; posterior margin of sternite VIII (Fig. 85) strongly convex, marginal setae moderately long, thin, and evenly spaced; median lobe of aedeagus (Figs 86-88) approximately $0.25 \mathrm{~mm}$ long; ventral process sinuate in lateral view, broad and apically of broadly triangular shape in ventral view; crista apicalis prominent in lateral view.
+ : posterior margin of sternite VIII (Fig. 90) broadly convex.

Comparative notes: Anaulacaspis eminens is distinguished from other small species of similar coloration (A. persica and allied species) only by the shape of the abdominal tergite VIII and by the morphology of the median lobe of the aedeagus, from the sympatric A. persica also by the paler coloration of the abdominal segments III-IV.

Distribution and natural history: The type specimens were collected in several localities in Hormozgan and Kerman provinces, South Iran, and in Afghanistan (Map 3). The altitudes range from 850 to $3360 \mathrm{~m}$. The specimens were collected partly on the banks of rivers, partly from under stones on stony slopes.

\section{Anaulacaspis gratilla (ERICHSON, 1839) \\ (Figs 121-131, Map 4)}

Falagria gratilla ERICHSON, 1839: $54 \mathrm{f}$.

Falagriola lutzi ReItTer, 1909: 75; syn. n.

Type material examined: Falagria gratilla: Syntype [damaged; head, pronotum, and abdominal segments V-X missing]: “5298 / gratilla Er., Mesopot. Helfer / Syntypus Falagria gratilla Erichson, 1839, labelled by MNB 2011 / Anaulacaspis gratilla (Erichson), det. V. Assing 2016" (MNB).

Falagriola lutzi: Lectotype $\sigma^{\star}$, present designation: "Margelan. Reitter. / coll. Reitter / Holotypus Falagriola Lutzi Reitter [curator label] / Falagriola lutzi m. 1908. / Falagria lutzi (Rtt.) ơ, V.I. Gusarov det. 1996 / Lectotypus ${ }^{\top}$ Falagriola lutzi Reitter, desig. V. Assing 2016 / Anaulacaspis gratilla (Erichson), det. V. Assing 2016" (HNHM). Paralectotypes: 3 i ㅇ: same data as lectotype.

Comment: The original description of Falagria gratilla is based on an unspecified number of syntypes from "Mesopotamia, Dr. Helfer" (ERICHson 1839). The sole syntype located in the Erichson collection at the MNB is severely damaged (only meso- and metathorax and base of abdomen left), which is why it is not designated as the lectotype. Falagriola lutzi was described in a footnote, based on an unspecified number of syntypes from “Transkaspien, Margelan” (ReitTer 1909). Four syntypes, a male and three females, were located in the collections of the HNHM. They had been dissected by V. Gusarov (Oslo), who attached (para-)lectotype labels to them without ever publishing the designation.

In view of the poor condition of the type specimen of Anaulacaspis gratilla, an interpretation of this species is problematic. Nevertheless, it is hypothesized to be conspecific with the type material of $A$. lutzi for three reasons. First, "Mesopotamia" is unlikely to host a species not treated in the course of the present revision. 
Second, material of only one Anaulacaspis species has been recorded from this region, and these specimens are conspecific with $A$. lutzi. Finally, a comparison of material of $A$. lutzi with what is left of the type specimen of A. gratilla revealed no morphological differences. Consequently, A. lutzi is placed in synonymy with A. gratilla.

Additional material examined: Ukraine: $4 o^{\top} o^{\lambda}, 4$ 우 우, Odessa, 28.IV., leg. Lgocki (NHMW, cAss).

Iran: Azarbayjan-e Gharbi: $60^{\top} 0^{\star}, 3$ 우 일 road TabrizMarand, $4 \mathrm{~km}$ S Ivand, $38^{\circ} 19^{\prime} \mathrm{N}, 46^{\circ} 08^{\prime} \mathrm{E}, 1560 \mathrm{~m}$, 28.VIII.2008, leg. Frisch \& Serri (MNB, cAss). Semnan: $40^{\star} 0^{\star}, 8$ 우 우, $17 \mathrm{~km}$ N Shahmirzad, $5 \mathrm{~km} \mathrm{~S}$ Chashm, $35^{\circ} 51^{\prime} \mathrm{N}, 53^{\circ} 18^{\prime} \mathrm{E}, 2040 \mathrm{~m}, 22 . \mathrm{V} .2006$, leg. Frisch \& Serri (MNB, cAss). Mazandaran: $10^{\star}$, Farahabad, $36^{\circ} 49^{\prime} \mathrm{N}$, $53^{\circ} 12^{\prime} \mathrm{E}, 9$. VII.1975, leg. Senglet (cAss).

Iraq: $2 \sigma^{\star} o^{x}, 2$ 우 우 , "Mesopotamien” (MNB).

Azerbaijan: $2 \sigma^{\star} \sigma^{\star}, 2$ ㅇ ㅇ, Ordubad [“Araxesthal”], leg. Leder \& Reitter (NHMW, cAss); 1 , Xanlar ["Helenendorf"], leg. Reitter (NHMW).

Uzbekistan: $3 \sigma^{\star} \sigma^{\star}, 1$ ㅇ, Marghilon [“Margelan”], leg. Staudinger, Reitter (MNB, NHMW, cAss); 1 ㅇ, Taškent (NHMW).

Kazakhstan: $10^{\star}$, Džambul (“Aulie Ata”) (MNB).

Afghanistan: $10^{\star}, 1$ 우, Kandahar, E Kandahar, $31^{\circ} 37^{\prime} \mathrm{N}$, 65³6'E, 31.VII.1975, leg. Senglet (cAss); $10^{*}$, Baghlan Prov., Doshi District, N Salang, Kilagi, 1200 m, 2.VIII.2010, leg. Reuter (cFel).
Locality not specified: 2 우, “Caucasus”, leg. Leder \& Reitter (NHMW); 1 ㅇ, “Turcmenien”, leg. Leder \& Reitter (NHMW).

Redescription: Body length $1.8-2.3 \mathrm{~mm}$; length of forebody $0.9-1.1 \mathrm{~mm}$. Coloration: head dark-brown; pronotum yellowish-red to reddish-brown; elytra reddish with a medio-lateral infuscate spot of very variable size, sometimes leaving only the humeral angles and the posterior margins of the elytra yellowish; abdomen dark-brown to blackish-brown, with segments I-II or I-III sometimes paler; legs pale-yellowish; antennae pale-brown to darkbrown, with antennomeres I-III yellowish.

Head approximately 1.1 times as broad as long, with more or less weakly pronounced sexual dimorphism; punctation very fine and sparse. Eyes longer than postocular region in dorsal view. Antenna approximately $0.9 \mathrm{~mm}$ long; antennomeres IV approximately as long as broad, $\mathrm{V}$ transverse, VI-X increasingly transverse and of gradually increasing width, $\mathrm{X}$ approximately twice as broad as long or nearly so.

Pronotum approximately 1.1 times as broad as long and as broad as head, with distinct sexual dimorphism.

Elytra 1.10-1.15 times as long as pronotum; punctation moderately dense and very fine. Hind wings fully developed.

Abdomen narrower than elytra; posterior margin of tergite VIII (Fig. 121, 128) truncate to weakly convex, postero-laterally with a (sometimes indistinct) cluster of long setae; marginal setae rather short and unmodified.

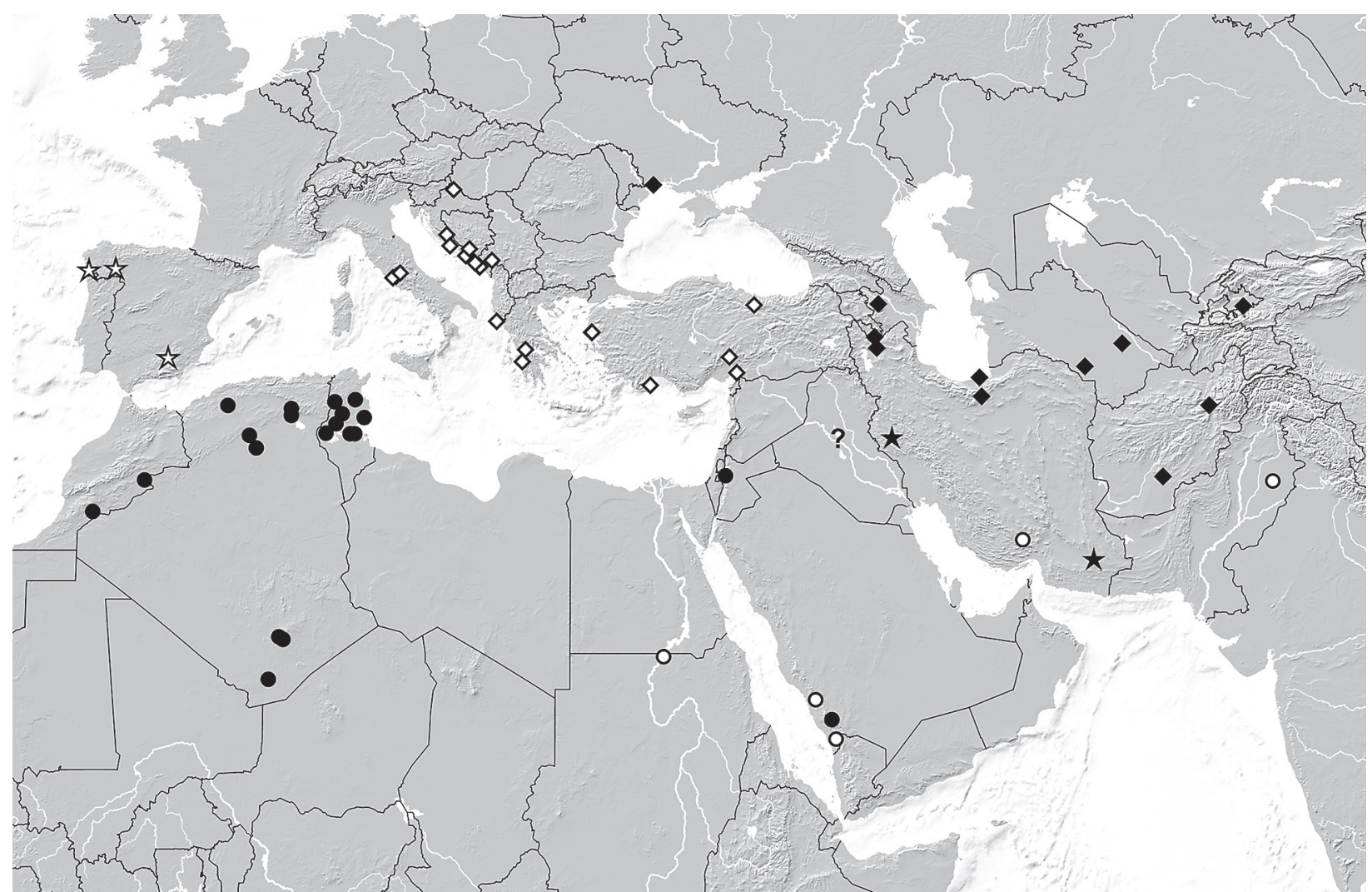

Map 4: Distributions of Anaulacaspis desertorum (black circles), A. naevula (white circles), A. iberica (white stars), A. laevigata (white diamonds), A. gratilla (black diamonds), and A. excisa (black stars) in the West Palaearctic and Middle Asia, based on revised records. The question mark indicates the records without specified locality of $A$. gratilla from Iraq. 
$\sigma^{*}$ : head somewhat flattened or weakly impressed in postero-median portion; pronotum with moderately fine and distinct punctation, in postero-median portion with extensive shallow impression; sternite VIII (Fig. 122, 129) distinctly transverse, posterior margin obtusely pointed in the middle; median lobe of aedeagus (Figs 123-126, 130-131) $0.30-0.36 \mathrm{~mm}$ long; ventral process short and strongly curved in lateral view, narrowly triangular and apically acute in ventral view; crista apicalis strongly developed, long and sinuate.

i : head not flattened in postero-median portion; pronotum without extensive impression and with extremely fine punctation; sternite VIII (Fig. 127) strongly transverse, posterior margin broadly convex, in the middle weakly concave.

Intraspecific variation: Like many other species of the genus, A. gratilla is subject to pronounced intraspecific variation of size, coloration, and other characters. The aedeagus, too, is rather variable, not only regarding size, but also with respect to the shape of the ventral process of the aedeagus. The primary and secondary sexual characters of a nanistic male from Azerbaijan are illustrated in Figs 128-131.

Comparative notes: Anaulacaspis gratilla is somewhat transitional between the species allied to A. nigra (in that it has the postero-lateral clusters of long thin setae on tergite VIII) and those allied to A. desertorum, with which it shares a similar shape of the male (not distinctly produced posteriorly) and female sternite VIII (weakly concave in the middle). This species is reliably distinguished from the highly similar and sympatric $A$. seclusa only by smaller average body size, the flat male head and the completely different shape of the ventral process of the aedeagus.

Distribution: The vast distribution of $A$. gratilla ranges from Ukraine, Azerbaijan, Iraq, and Iran into Middle Asia (Map 4), where it appears to be rather common. The altitudes indicated on the labels range from 1200 to $2040 \mathrm{~m}$.

\section{Anaulacaspis naevula (ERICHSON, 1839)}

(Figs 93-101, Map 4)

Falagria naevula ERICHSON, 1839: 55.

Falagria nilotica КocH, 1936: 209.

Myrmecopora (Ilyusa) elegans CAMERON, 1944: 52; preoccupied.

Anaulacaspis elegansides Newton, 2015: 10; replacement name; syn. $\mathrm{n}$.

Type material examined: F. naevula: Lectotype $\sigma^{*}$ : “Aegypt, Ehrbrg., Nr. 5299 / G. Fagel elig. 1968, Falagria naevula Erichson, Lectotype / naevula Er., XXXV. XLVII. Ehrb. / Anaulacaspis naevula (Erichson), det. V. Assing 2016” (MNB). Paralectotypes: $1 \sigma^{\star}$ [in poor condition: head and legs partly missing], 1 \% [antennae and most legs missing]: "Aegypt, Ehrbrg., Nr. 5299 / Paralectotypus Falagria naevula Erichson, 1830, labelled by MNB 2011" (MNB).

M. elegans: Holotype $0^{*}:$ "Under fallen leaves in tomato field, Lyallpur, 22.6.1939, H. R. Ghani / Myrmecopora elegans Cam., Type / Govt. Entomologist Punjab C13 / Pres. by Imp. Inst. Ent. B.M. 1940-119 / Type [round curator label] / Holotype Anaulacaspis elegans (Cameron), det. V. Assing 1997 / Anaulacaspis naevula (Erichson), det. V. Assing 2016" (BMNH). Paratype + : "In Karela field, students farm, Lyallpur, M.A. Ghani 13.6.39 / Govt. Entomologist Punjab ... C 13 / under dead leaves on moist soil / Myrmecopora elegans Cam. Cotype / elegans Cam. / M. Cameron. Bequest. B.M.1955-147. / Anaulacaspis klapperichi (Coiffait), det. V. Assing 2016” (BMNH).

Comment: The original description of Falagria naevula is based on an unspecified number of syntypes from "Aegypto" (ERICHSON, 1830). FAGEL (1969) designated a male as the lectotype and placed Falagria nilotica Косн, 1936 (type locality: Wadi Halfa; today in Sudan) in synonymy with $F$. naevula.

CAMERon (1944) described Myrmecopora elegans based on an unspecified number of type specimens from "Lyallpur. Under dead leaves on moist soil". In stating "Type in British Museum (Nat. Hist.)" he designated a holotype. The male holotype and a female paratype were located in the Cameron collection at the BMNH. Both specimens had been studied by Assing (1997), who moved this species from Myrmecopora to Anaulacaspis. The preoccupied name Anaulacaspis elegans (CAMERON, 1944) was recently replaced with the nomen novum A. elegansides by Newton (2015). The paratype was studied prior to the holotype and had already been returned when the latter was found. Based on an examination of the paratype, A. elegansides was at first believed to be conspecific with A. klapperichi (hence the identification label). An examination of the holotype, however, revealed that A. elegansides is in fact conspecific with $A$. naevula.

Additional material examined: Iran: $7 \sigma^{\wedge} \sigma^{*}, 14$ 우 [mostly teneral], Hormozgan province, $9 \mathrm{~km}$ SW Hajiabad, $28^{\circ} 15^{\prime} \mathrm{N}, 55^{\circ} 51^{\prime} \mathrm{E}, 840 \mathrm{~m}, 22 . \mathrm{IV} .2006$, leg. Frisch \& Serri (MNB, cAss).

Saudi Arabia: $4 \sigma^{\star} \sigma^{\star}, 1$ ㅇ [ $1 \sigma^{\star}$ teneral], Al Urdiyah, Wadi Gonouna, $19^{\circ} 26^{\prime} \mathrm{N}, 41^{\circ} 36^{\prime} \mathrm{E}, 350 \mathrm{~m}, 12 . \mathrm{V} .2011$, leg. Sharaf (cAss); 1 ㅇ, Wadi Qasi, $40 \mathrm{~km}$ from Sabia, $17.25^{\circ} \mathrm{N}$, $42.97^{\circ} \mathrm{E}, 270 \mathrm{~m}, 12 . X I .2012$, leg. Sharaf (cAss).

Sudan: $2 \sigma^{\star} o^{\star}, 3$ ㅇ + , bank of Nile river near Wadi Halfa, IV.1930, leg. Kock (NHMW, cAss).

Redescription: Body length 2.0-2.5 mm; length of forebody $0.95-1.2 \mathrm{~mm}$. Coloration: head yellowish-brown to brown; pronotum yellowish-red to reddish; elytra yellowish with a moderately extensive, diffusely delimited, and usually weakly pronounced medio-lateral infuscate spot; abdomen: segments III-IV yellowish to 
reddish-yellow, tergites V-VIII infuscate, often with the anterior and posterior, sometimes also the lateral margins more or less extensively yellowish to reddish-yellow; legs yellowish; antennae yellowish-brown to brown with the basal 2-4 antennomeres yellowish.

Head approximately 1.15 times as broad as long, with more or less pronounced sexual dimorphism. Eyes much longer than postocular region in dorsal view. Antenna 0.8-0.9 mm long; antennomeres IV weakly oblong, V weakly oblong or approximately as long as broad, VI approximately as long as broad or weakly transverse, VII-X of gradually increasing width and increasingly transverse, $\mathrm{X}$ approximately 1.5 times as broad as long.

Pronotum 1.05-1.15 times as broad as long and slightly narrower than head, with pronounced sexual dimorphism.

Elytra approximately as long as, or slightly shorter than, pronotum; punctation rather dense and fine. Hind wings fully developed.

Abdomen as broad as, or slightly narrower than, elytra; punctation dense and fine; interstices without microsculpture; posterior margin of tergite VIII (Fig. 91) truncate to weakly convex, with moderately long and thin marginal setae.

$\sigma^{\star}$ : head with more or less distinct and more or less extensive median impression, dorsal surface anteriorly and laterally with distinct punctation; pronotum with pronounced, deep and broad median impression in posterior two-thirds, punctation dense, distinct, and somewhat asperate; posterior margin of sternite VIII (Fig. 92) obtusely angled in the middle and with thin marginal setae; median lobe of aedeagus (Figs 93-100) 0.32-0.37 mm long; ventral process of broadly triangular shape in ventral view, apex with or without small incision (ventral view).

o : dorsal surface of head convex in cross-section and with very fine punctation; pronotum with shallow and rather small postero-median impression at most and with dense and fine punctation; posterior margin of sternite VIII (Fig. 101) weakly concave in the middle.

Intraspecific variation: As is usually the case with widespread species, some characters are subject to pronounced variation. In the specimens from Saudi Arabia, the male head has a pronounced and deep median impression, and the aedeagus is at the low end of the size range (median lobe $0.32-0.33 \mathrm{~mm}$ long), where as in material seen from other regions the impression on the male head is shallow and more extensive, and the aedeagus is generally somewhat larger. Moreover, the ventral process of the aedeagus is apically weakly incised in ventral view in males from Iran and Pakistan, while it is simply acute in the type specimens of A. naevula and in specimens from Saudi Arabia.

Comparative notes: Based on the similar modifications of the male pronotum, the similar shapes of tergite and sternite VIII, as well as particularly on the similar morphology of the median lobe of the aedeagus, A. naevula is closely allied to A.desertorum and A. seclusa. The species is characterized by small body size, the coloration, a pronounced sexual dimorphism of the head and pronotum, and by the shape of the median lobe of the aedeagus. It additionally differs from the similarly pale-coloured and small $A$. persica and allied species by the completely different shape of tergite VIII (posteriorly without pronounced median process), as well as by the shape of the pronotum (less strongly tapering posteriorly).

Distribution and natural history: Since A. naevula has largely been misinterpreted in the past, nearly all literature records are erroneous. Confirmed records with specified localities are currently known from Sudan, Saudi Arabia, South Iran, and Pakistan (Map 4). The examined non-type material was collected in wadis and on a river bank at altitudes of $270-840 \mathrm{~m}$.

\section{Anaulacaspis desertorum (FAUVEL, 1898) \\ (Figs 102-107, Map 4)}

Falagria desertorum FAUveL, 1898: 106.

Type material examined: Lectotype $\sigma^{\pi}$ [dissected by R. Pace]: "Sfax, Tunisie / Coll. et det. A. Fauvel, Falagria desertorum Fauvel, R.I.Sc.N.B. 17.479 / Ex-Typis / G. Fagel elig., 1968, Falagria desertorum Fauv., Lectotype / vidit R. Pace 1985 / Anaulacaspis desertorum (Fauvel), det. V. Assing 2016” (IRSNB). Paralectotypes: 2 ㅇ ㅇ: "Desertorum Fvl. Tunisie / R.I.Sc.N.B. 17.479, Falagria, Coll. et det. A. Fauvel / Ex-Typis / Syntype / Falagria desertorum Fvl., det. R. Pace 1985” (IRSNB); 1 \% : “Tilremt, Algérie / coll. et det. A. Fauvel, Falagria desertorum Fauvel, R.I.Sc.N.B. 17.479 / Ex-Typis / Falagria desertorum Fvl., det. R. Pace 1985" (IRSNB); 1 o [dissected by R. Pace]: "Bled Thala, Tunisie / coll. et det. A. Fauvel, Falagria desertorum Fauvel, R.I.Sc.N.B. 17.479 / Ex-Typis / Falagria desertorum Fvl., det. R. Pace 1985" (IRSNB); 1 ㅇ: "Laghouat, 5 / coll. et det. A. Fauvel, Falagria desertorum Fauvel, R.I.Sc.N.B. 17.479 / Ex-Typis / Falagria desertorum Fvl., det. R. Pace 1985" (IRSNB); 1 đ [dissected by R. Pace]: “Tiout, Oranais / coll. et det. A. Fauvel, Falagria desertorum Fauvel, R.I.Sc.N.B. 17.479 / Ex-Typis / Falagria desertorum Fvl., det. R. Pace 1985” (IRSNB); 1 ㅇ: “Nza-ben-Rzig, Oued-Rirh, Algérie / El Kantara, 3 / coll. et det. A. Fauvel, Falagria desertorum Fauvel, R.I.Sc.N.B. 17.479 / Ex-Typis / Falagria desertorum Fvl., det. R. Pace 1985" (IRSNB); $20^{\top} 0^{\top}$ [teneral]: "Biskra, 5 / Desertorum Fvl. / R.I.Sc.N.B. 17.479, Falagria, Coll. et det. A. Fauvel / Ex-Typis / Falagria desertorum Fvl., det. R. Pace 1985" (IRSNB).

Comment: The original description is based on an unspecified number of syntypes from "Algérie, Tunisie" (Fauvel 1898). Fagel (1969) designated a male from Tunisia as the lectotype. 
Additional material examined: Morocco: $1 \sigma^{\top}$, Erfoud, Bou Gafer, V.1975, leg. Curti (cAss); 1 ơ $^{\star}$ Tata, Aka Iguirene, UV light, 12.V.1915, leg. Miessen (cTro); 1 우, Ouarzazate, 23.V.1986, leg. Fongond (cTro).

Algeria: $4 \sigma^{\star} o^{\star}, 2$ 우 우, Biskra (NHMW, cAss); 1 우, Takaraba, Oued Tamanghanet, 12.IV.1948, leg. Hollande (cTro); 1 ㅇ, Hoggar, Tesselite Lehete, IV.1961, leg. Hollande (cTro); $3 o^{\star} o^{\star}$, Hoggar, Tela N'telahedit, IV.1961, leg. Hollande (cTro); $1 \sigma^{\star}, 1$ † , Djebel Metlilli, gorges d'El Kantara, V.1954, leg. Fagel (MHNG).

Tunisia: 1 \% , Jerid, $4 \mathrm{~km}$ NE Tozeur, 30-40 m, light trap, 21.X.2009, leg. Lehmann (cAss); 3 우 ㅇ, Gafsa, Seldja gorge, XII.1980, leg. Frank (cAss); $2 \sigma^{\star} o^{\star}, 2$ ㅇ + , Gafsa,

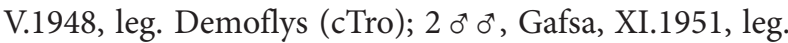
Demoflys (cAss); 1 o , Tozeur, VI.1953, leg. Demoflys (cTro); 2 우 오 Kairouan, XI.1936, leg. Normand (cTro); 1 ơ, Bir el Hafey, VI.1947, leg. Demoflys (cTro); 1 우, Gabès, I.1946, leg. Demoflys (cTro); 1 o , El Hamma, I. 1949, leg. Demoflys (cTro).

Jordan: $10^{4}, 6$ 우 우 [2우 우 teneral], Madaba, Wadi Manshala, $31^{\circ} 41^{\prime} \mathrm{N}, 35^{\circ} 35^{\prime} \mathrm{E},-280 \mathrm{~m}, 1 . I I I .2014$, leg. Meybohm (cAss); 4 우 오, Madaba, Wadi Manshala, $31^{\circ} 41^{\prime} \mathrm{N}, 35^{\circ} 35^{\prime} \mathrm{E},-370 \mathrm{~m}$, 4.III.2015, leg. Meybohm \& Hetzel (cAss, cFel).

Saudi Arabia: $20^{\star} o^{*}$, Khamis Mushayt, Wadi Bishah, $18^{\circ} 20^{\prime} \mathrm{N}, 42^{\circ} 42^{\prime} \mathrm{E}, 1990 \mathrm{~m}, 27 . \mathrm{IV} .2011$, leg. Sharaf (cAss).

Redescription: Body length 2.1-2.6 mm; length of forebody 1.1-1.3 mm. Coloration: head dark-brown to black; pronotum reddish to reddish-brown; elytra yellowish, with an extensive medio-lateral dark spot usually leaving only the posterior margins, the suture, and the humeral portions yellow; abdomen dark-brown to black, with segments III and IV reddish-yellow; legs brown with yellow tarsi; antennae brown to blackish-brown, with the basal 2-3 antennomeres reddish.

Head approximately 1.15 times as broad as long, without distinct sexual dimorphism; punctation moderately fine, distinct, and rather dense. Eyes distinctly longer than postocular region in dorsal view. Antenna $0.8-1.0 \mathrm{~mm}$ long; antennomeres IV-V as long as broad or oblong, VI-X of gradually increasing width and increasingly transverse, $\mathrm{X}$ approximately 1.5 times as broad as long. Pronotum 1.05-1.10 times as broad as long and approximately as broad as head, with pronounced sexual dimorphism; punctation rather dense and distinct.

Elytra slightly longer than pronotum; punctation rather dense and fine. Hind wings fully developed.

Abdomen narrower than elytra; punctation more or less regularly distributed; posterior margin of tergite VIII (Fig. 102) weakly convex, with relatively short and thin marginal setae.

$\sigma^{7}$ : pronotum with extensive median impression and with dense and distinct punctation; posterior margin of sternite VIII (Fig. 103) strongly convex to obtusely pointed and with long thin marginal setae; median lobe of aedeagus (Figs 104-107) approximately $0.33-0.35 \mathrm{~mm}$ long, without conspicuous modifications.
ㅇ: pronotum with small and shallow impression in postero-median portion, this impression anteriorly extending into a short narrow sulcus, punctation slightly less distinct than in male; posterior margin of sternite VIII broadly convex, in the middle usually weakly concave.

Comparative notes: Anaulacaspis desertorum differs from the closely related $A$. naevula by paler coloration and above all by the different shape of the ventral process of the aedeagus in ventral and in lateral view, from the sympatric and syntopic $A$. elegans by a usually more paleyellowish posterior margin of the elytra, shorter antennae, a more transverse pronotum, more densely punctate elytra and abdomen, unmodified setae at the posterior margin of tergite VIII, and a median lobe of the aedeagus with a shorter and differently shaped ventral process. For characters distinguishing $A$. desertorum from the closely related and similarly coloured $A$. seclusa see the redescription in the following section.

Distribution and natural history: The known distribution ranges from Morocco across North Africa into the Middle East (Jordan, Saudi Arabia) (Map 4). The altitudes indicated on the labels range from -370 to $1990 \mathrm{~m}$. Two specimens were collected with a light trap. Two specimens found in early March are teneral.

\section{Anaulacaspis seclusa (FAgEL, 1969) \\ (Figs 108-120, Map 6)}

Melagria seclusa FAgEL, 1969: $21 \mathrm{f}$.

Falagria (Melagria) klapperichi CoIfFAIt, 1982: 88 f.; syn. n.

Type material examined: M. seclusa: Holotype o": “Turkmenistan, Baïram-Ali, 11-24.V.1959 / G. Fagel det., seclusa n. sp. (Melagria) / R.I.Sc.N.B., I. G. 24885 / Type / Falagria seclusa Fagel, det. R. Pace 1985 / Anaulacaspis seclusa (Fagel), det. V. Assing 2016” (IRSNB). Paratypes: 1 ㅇ: same data as holotype (IRSNB); $10^{\star}$ [teneral]: "Turkmenistan, Repetek, 8-10.V.1952/ R.I.Sc.N.B., I. G. 248851 / G. Fagel det., seclusa n. sp. (Melagria) / Paratype / Falagria seclusa Fagel, det. R. Pace 1985" (IRSNB); 1 o: :Persia merid., 1862-63, Coll. P. Doria / Coll. et det. A. Fauvel, Falagria naevula Er., R.I.Sc.N.B. 17.4797 / G. Fagel det. 1968, Falagria seclusa n. sp. / Paratype / Falagria seclusa Fagel, det. R. Pace 1985” (IRSNB).

F. klapperichi: Holotype $\sigma^{\top}$ : “ $\sigma^{\star} /$ 14.1.53, S-Afghanistan / J. Klapperich, Scham - Schir - Ror, 950 m, b. Kandahar / Falagria klapperichi nov. spec. / Typus Falagria klapperichi O. Scheerpeltz / Falagria klapperichi H. Coiffait 1981 / Holotypus / Museum Koenig Bonn / Anaulacaspis seclusa (Fagel), det. V. Assing 2016" (ZFMK). Paratypes: 1 웅 "Allotypus / Typus Falagria klapperichi O. Scheerpeltz / + / 14.1.53, S-Afghanistan / J. Klapperich, Scham - Schir Ror, 950 m, b. Kandahar / H. Coiffait det. 1981 / Museum Koenig Bonn” (ZFMK); 1 ㅇ: “o / 14.1.53, S-Afghanistan 
/ J. Klapperich, Scham - Schir - Ror, 950 m, b. Kandahar / Cotypus Falagria Klapperichi O. Scheerpeltz / Falagria Klapperichi H. Coiffait 1981 / Museum Koenig Bonn” (MNHNP).

Comment: The original description is based on a male holotype and a male paratype from "Turkmenistan : Baïram-Ali", a female paratype from "Turkmenistan : Repetek", and a male paratype from "Persia merid." (FAGEL 1969). Falagria klapperichi was described based on a male holotype and two female paratypes from "Sud Afghanistan, Scham-Schir-Ror près Kandahar, 950 m, 14-I-53, J. Klapperich leg." (CoIffait 1982). An examination of the type specimens of both names revealed that they are conspecific. The aedeagus of the holotype (Figs 115-116) is at the low end of the size range, but otherwise no characters were observed suggesting that A. klapperichi should represent a distinct species. Hence the synonymy proposed above.

Additional material examined: Armenia: 1 i [identification doubtful], Ecmiadzin, 9.III.1916, leg. Eichler (NHMW).

Iran: Azarbayjan-e Gharbi: $2 \sigma^{\star} \sigma^{\star}, 1$ 우, N Takab, $3 \mathrm{~km}$ NE Takht-e-Soleyman, $36^{\circ} 38^{\prime} \mathrm{N}, \quad 47^{\circ} 14^{\prime} \mathrm{E}, 2270 \mathrm{~m}$, 8.IX.2008, leg. Frisch \& Serri (MNB, cAss). Kordestan: $2 o^{\star} o^{\star}, 2$ ㅇ 우, $27 \mathrm{~km}$ SW Saqqez, $2 \mathrm{~km}$ SW Mir Deh, $36^{\circ} 08^{\prime} \mathrm{N}, 46^{\circ} 03^{\prime} \mathrm{E}, 1600 \mathrm{~m}$, 3.IX.2008, leg. Frisch \& Serri (MNB, cAss); $10^{\star}$, road Sanandaj - Divandarreh, $21 \mathrm{~km} \mathrm{~S}$ Divandarreh, $35^{\circ} 46^{\prime} \mathrm{N}, 47^{\circ} 05^{\prime} \mathrm{E}, 1890 \mathrm{~m}$, 6.IX.2008, leg. Frisch \& Serri (cAss). Qazvin: 1 우 Ab Garm, 354ㅇ' $\mathrm{N}, 49^{\circ} 23^{\prime} \mathrm{E}, 1500 \mathrm{~m}, 21 . \mathrm{VI} .2004$, leg. Frisch (MNB). Golestan: $60^{\top} o^{\star}, 5$ 우 우 [1 $0^{\star}$ teneral $]$, Golestan National Park, Tang Rah, 37 $24^{\prime} \mathrm{N}, 55^{\circ} 47^{\prime} \mathrm{E}$, $490 \mathrm{~m}, 4 . \mathrm{VI} .2006$, leg. Frisch \& Serri (MNB, cAss). North Khorasan: $4 \sigma^{\star} \sigma^{\star}, 3$ ㅇ 9 [partly teneral], road Shirvan - Quchan, 24 km SSW Faruj, Garmab, 37 $03^{\circ} \mathrm{N}$, $58^{\circ} 07^{\prime} \mathrm{E}, 1710 \mathrm{~m}, 1 . \mathrm{VI} .2006$, leg. Frisch \& Serri (MNB, cAss). Razavi Khorasan: $5 o^{\star} o^{\star}, 3$ 우 우 [1 $\sigma^{\star}, 1$ 우 teneral], 20 km NW Torbat-e, Heydariyeh, Senobar, 35² $26^{\prime} \mathrm{N}$, $59^{\circ} 06^{\prime} \mathrm{E}, 1730 \mathrm{~m}, 29 . \mathrm{V} .2006$, leg. Frisch \& Serri (MNB, cAss). Semnan: $10^{\star}$, Firuzkuh-Semnan, $27 \mathrm{~km} \mathrm{NW}$ Semnan, Aftar, $35^{\circ} 34^{\prime} \mathrm{N}, 5^{\circ} 09^{\prime} \mathrm{E}, 1580 \mathrm{~m}, 21 . \mathrm{V} .2006$, leg. Frisch \& Serri (MNB). Ilam: 1 오, Sarableh Kuhdasht road, NW Sarneh, $33^{\circ} 41^{\prime} \mathrm{N}, 47^{\circ} 04^{\prime} \mathrm{E}, 890 \mathrm{~m}$, 17.X.2011, leg. Frisch (cAss). Lorestan: $50^{\star} 0^{\star}, 5$ 우 우, 35 km E Kuhdasht, Kashkan, 3335'N, 475'ㄹ, 1010 m, 17.X.2011, leg. Frisch (MNB, cAss). Chahar Mahal-oBakhtiyari: $20^{\top} o^{\star}, 7$ 우, Izeh-Lordegan, $7 \mathrm{~km}$ SW Manj, 31 $34^{\prime} \mathrm{N}, 50^{\circ} 34^{\prime} \mathrm{E}, 1250$ m, 20.X.2011, leg. Frisch (MNB, cAss). Kerman: $10^{\star}, 1$ ㅇ, Rayen-Darb Behesht, $2 \mathrm{~km}$ W Goruh, $29^{\circ} 21^{\prime} \mathrm{N}, 57^{\circ} 21^{\prime} \mathrm{E}, 2700 \mathrm{~m}, 28 . V .2010$, leg. Frisch (MNB, cAss); 1 क [teneral], Darb BeheshtJiroft, $50 \mathrm{~km} \mathrm{~N}$ Jiroft, $10 \mathrm{~km} \mathrm{~N}$ Delfard Goruh, 2904'N, $57^{\circ} 33^{\prime} \mathrm{E}, 2650 \mathrm{~m}, 28 . \mathrm{V} .2010$, leg. Frisch (MNB); $1 \mathrm{o}^{\mathrm{T}}$, 1 ㅇ, Banan mountains, pass Mahan-Sirch, $30^{\circ} 12^{\prime} \mathrm{N}$, $57^{\circ} 24^{\prime}$ E, 2800 m, 20.V.2010, leg. Frisch \& Serri (MNB); $30{\sigma^{\star}}^{\star} \sigma^{\star}, 16$ 우 우 [partly teneral], Kuhpaye, $30^{\circ} 29^{\prime} \mathrm{N}$,
57019'E, 1800 m, 29.IV.2007, leg. Frisch \& Serri (MNB, cAss); $1 \sigma^{\star}$, Keyber mountains, Khabr, $28^{\circ} 49^{\prime} \mathrm{N}, 56^{\circ} 20^{\prime} \mathrm{E}$, 2060 m, 21.IV.2006, leg. Frisch \& Serri (cAss); $10^{\text {t }}$, 1 을, Anar - Shahr-e-Babak road, Javazm, 30³1'N, 550'ㄹ, 2180 m, 18.V.2010, leg. Frisch \& Serri (MNB, cAss); $10^{\star}$, pass Mahan-Sirch, $30^{\circ} 12^{\prime} \mathrm{N}, \quad 57^{\circ} 24^{\prime} \mathrm{E}$, 2800 m, 20.V.2010, leg. Frisch \& Serri (cAss); 1 ㅇ, Darb Behesht-Jiroft, $50 \mathrm{~km} \mathrm{~N}$ Jiroft, $10 \mathrm{~km}$ N Delfard Goruh, $29^{\circ} 04^{\prime} \mathrm{N}, 57^{\circ} 33^{\prime} \mathrm{E}, 2650 \mathrm{~m}, 28$. V.2010, leg. Frisch \& Serri (cAss). Fars: 1 으, road Estahban-Darab, $23 \mathrm{~km} \mathrm{NW}$ Darab, $28^{\circ} 53^{\prime} \mathrm{N}, 54^{\circ} 24^{\prime} \mathrm{E}, 24 . I V .2006$, leg. Frisch \& Serri (MNB). Hormozgan: $2 \sigma^{\star} \sigma^{\star}, 5$ 우 우 W Hajiabad, Dar Agah, 282' $\mathrm{N}, 55^{\circ} 43^{\prime} \mathrm{E}, 1110 \mathrm{~m}$, 22.IV.2006, leg. Frisch \& Serri (MNB); 1 i [slightly teneral], 9 km SW Hajiabad, $28^{\circ} 15^{\prime} \mathrm{N}, 55^{\circ} 51^{\prime} \mathrm{E}, 840 \mathrm{~m}, 22 . I V .2006$, leg. Frisch \& Serri (MNB). Sistan va Baluchestan: 1 우, Iranshar

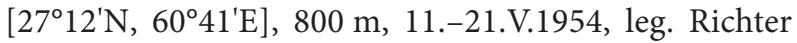
\& Schäuffele (NHMW). Locality not specified or not identified:1 $\circ$, "Persien" (NHMW).

Afghanistan: $10^{\star}, 1$ ㅇ, Kandahar, W Kandahar, $31^{\circ} 37^{\prime} \mathrm{N}$, 653'ㄹ [sic], 31.VII.1975, leg. Senglet (cAss).

Turkmenistan: $1 \sigma^{\top}$, Kov-Ata, $110 \mathrm{~km}$ NW Ashchabad, 10.V.1989, leg. Wrase (cAss).

Redescription: Body length 2.1-2.9 mm; length of forebody 1.1-1.4 mm. Tergite VIII as in Figs 108, 113, 119. Highly similar to $A$. desertorum, except as follows:

Pronotum on average more slender and with less pronounced sexual dimorphism; infuscate mediolateral spot of elytra on average less extensive.

$\sigma^{*}$ : pronotum with shallower and less extensive median impression; posterior margin of sternite VIII (Figs 109,114 ) obtusely pointed in the middle; median lobe of aedeagus (Figs 110-111, 115-118) 0.32-0.38 mm long; ventral process apically more slender in lateral view.

ㅇ: posterior margin of sternite VIII (Figs 112, 120) distinctly concave in the middle.

Intraspecific variation: The coloration is subject to pronounced intraspecific variation. In dark-coloured specimens, the body is practically completely black, except for the yellowish posterior margin of the elytra and the brownish humeral angles and suture, and the legs and the basal antennomeres are brown to darkbrown. Moreover, the aedeagus is quite variable in size and shape: the ventral process may be more or less strongly curved and more or less slender in lateral view (Figs 110-111, 115-118).

Distribution and natural history: Male-based records are currently known from Iran, Turkmenistan, and Afghanistan (Map 6). The altitudes range from 490 to $2800 \mathrm{~m}$. Teneral specimens were collected in April, May, and June. 


\section{Anaulacaspis cristata spec. nov.}

(Figs 132-135, Map 5)

Type material: Holotype $0^{\star}:$ "PAK, Northern Areas, Ghizar valley, Thiee, $36.0229^{\circ} \mathrm{N}, 74.1681^{\circ} \mathrm{E}, 5.7 .2007$ $1760 \mathrm{~m}$, leg. H. Mühle / Holotypus ơ Anaulacaspis cristata sp. n., det. V. Assing 2016" (MNB).

Etymology: The specific epithet is an adjective derived from the Latin noun crista. It alludes to the enormous crista apicalis of the aedeagus.

Description: Body length $2.3 \mathrm{~mm}$; length of forebody $1.2 \mathrm{~mm}$. Coloration: head pale reddish-brown; pronotum reddish; elytra yellowish, with a rather pale and diffusely delimited medio-lateral infuscate spot; abdomen reddish with blackish segments VI and VII; legs yellowish; antennae brown with the basal three antennomeres pale-reddish.

Head approximately 1.1 times as broad as long, probably with weak sexual dimorphism. Eyes indistinctly longer than postocular region in dorsal view. Antennae: antennomere IV weakly oblong; antennomeres V-X of gradually increasing width and increasingly transverse, $\mathrm{X}$ approximately 1.5 times as broad as long.

Pronotum approximately 1.1 times as broad as long and approximately as broad as head, evidently with distinct sexual dimorphism.

Elytra approximately as long as pronotum; punctation dense and very fine. Hind wings present.

Abdomen narrower than elytra; punctation distinct; tergite VIII (Fig. 132) rather weakly transverse, posterior margin truncate, postero-laterally without a cluster of long thin setae on either side, marginal setae unmodified and moderately long.

$\sigma^{7}$ : head with distinct punctation and with weakly pronounced median impression posteriorly; pronotum with extensive and distinct median impression in posterior two-thirds and with dense and distinct punctation; posterior margin of sternite VIII (Fig. 133) indistinctly, obtusely pointed in the middle; median lobe of aedeagus (Figs 134-135) $0.35 \mathrm{~mm}$ long; ventral process narrow, short (in relation to capsule), and weakly curved in lateral view, of triangular shape in ventral view; crista apicalis enormous.

†: unknown.

Comparative notes: As can be inferred from the shapes and chaetotaxy of tergite and sternite VIII, as well as from the general morphology of the aedeagus, A. cristata belongs to the A. naevula group. Among the species of this group, it is characterized particularly by the morphology of the aedeagus, above all the enormous crista apicalis.

Distribution: The type locality is situated in North Pakistan (Map 5) at an altitude of $1760 \mathrm{~m}$.

\section{Anaulacaspis excisa spec. nov.}

(Figs 136-141, Map 4)

Type material: Holotype $\sigma^{\star}$ : "IRAN, Ilam Province, Sarableh - Kuhdasht: NW Sarneh, 890 m, N 3341'04" E 04703'43", 17.10.2011, leg. Frisch / Holotypus $0^{\star}$ Anaulacaspis excisa sp. n., det. V. Assing 2016" (MNB).

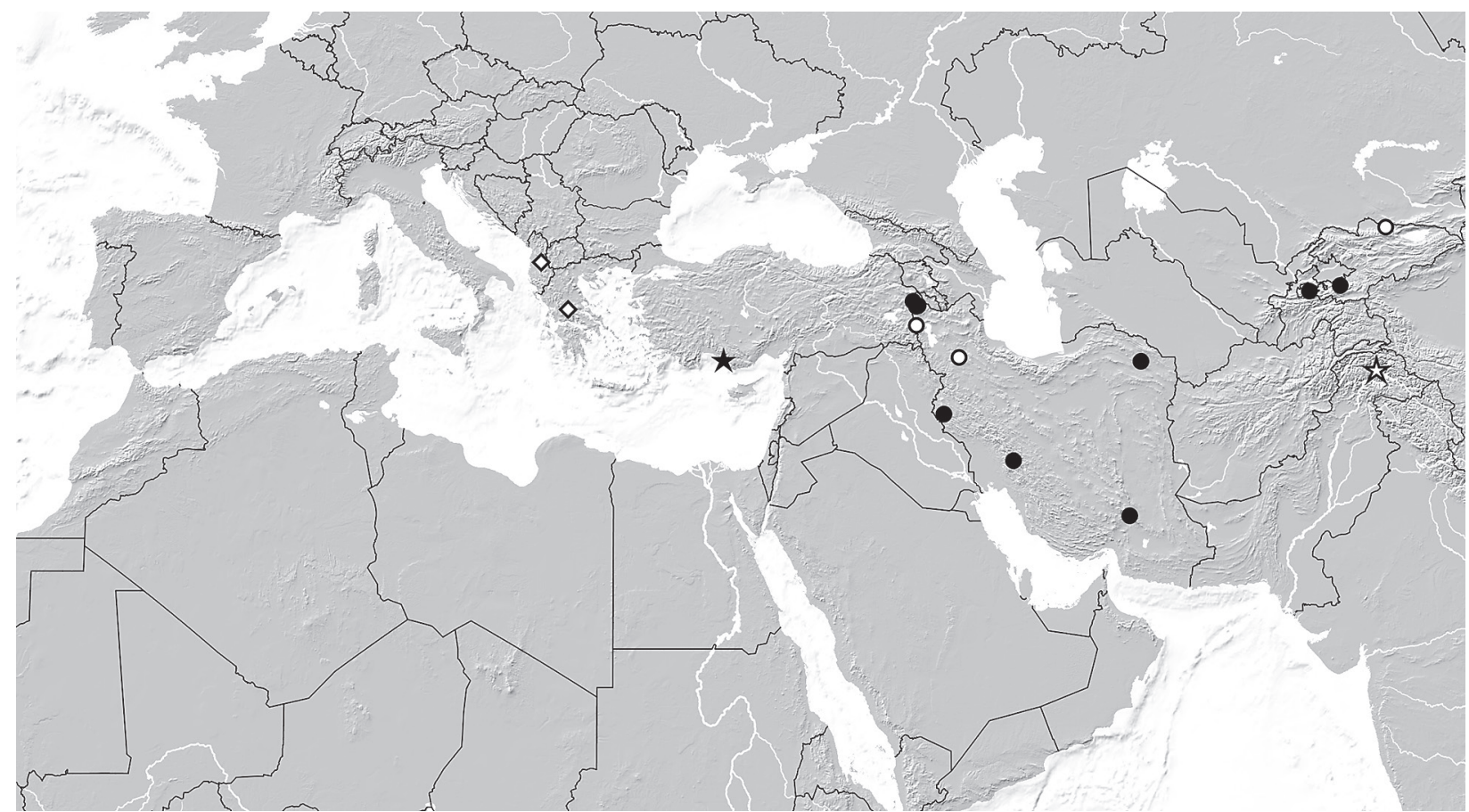

Map 5: Distributions of Anaulacaspis flavomarginata (white diamonds), A. pamphylica (black star), A. inexpectata (white circles), and A. persica (black circles) in the West Palaearctic and Middle Asia, based on revised records. 
Paratypes: $10^{*}, 2$ 우: same data as holotype (MNB, cAss); $1 \sigma^{*}$ : “ $\sigma^{*} / \mathrm{SO}$. Persien, Iranshar $\left[27^{\circ} 12^{\prime} \mathrm{N}, 60^{\circ} 41^{\prime} \mathrm{E}\right]$ / naevula / ex coll. Scheerpeltz" (NHMW).

Etymology: The specific epithet is the past participle of the Latin verb excidere (to cut out) and alludes to the conspicuous median excision of the posterior margin of tergite VIII.

Description: Body length 2.7-3.2 mm; length of forebody $1.3-1.5 \mathrm{~mm}$. Coloration: head blackish-brown to black; pronotum brown to blackish-brown; elytra with the anterior portion reddish to reddish-brown, the median portion (except for the suture) extensively blackish, and the posterior margin broadly pale-yellowish, the yellowish coloration extending to middle at suture; abdomen blackish-brown to black, with segments III and IV reddish-brown; legs dark-brown, except for the paler tarsi and femoral bases; antennae blackish-brown, with the basal 3-4 antennomeres brown.

Head approximately 1.15 times as broad as long, with distinct sexual dimorphism. Eyes slightly longer than postocular region in dorsal view. Antenna slender, $1.2-1.3 \mathrm{~mm}$ long; antennomeres IV-VII distinctly oblong, VIII-IX approximately as long as broad, and X weakly transverse.

Pronotum 1.10-1.15 times as broad as long and approximately as broad as, or slightly narrower than head, with pronounced sexual dimorphism.

Elytra slightly shorter than pronotum; punctation dense and very fine. Hind wings fully developed.

Abdomen approximately as broad as elytra; punctation dense, fine, and nearly regularly distributed; posterior margin of tergite VIII (Figs 136, 140) with weakly pronounced sexual dimorphism, posterior margin concave in the middle, marginal setae short, middle of posterior margin without marginal setae.

$\mathrm{o}^{\top}$ : head extensively depressed or weakly impressed, punctation distinct; pronotum with pronounced, deep, broad, and long median impression, punctation dense, distinct, and somewhat asperate; posterior margin of tergite VIII (Fig. 136) with broad and moderately deep median concavity; posterior margin of sternite VIII (Fig. 137) obtusely pointed in the middle, with thin and dense marginal setae; median lobe of aedeagus (Figs 138-139) $0.40-0.43 \mathrm{~mm}$ long; ventral process apically curved, nearly angled in lateral view, broadly triangular and apically acute in ventral view.

क: head not depressed or impressed, punctation distinct, but finer than in male; pronotum with very shallow median impression at most, punctation dense and distinct, but less so than in male and not asperate; posterior margin of tergite VIII (Fig. 140) with small and shallow median concavity; posterior margin of sternite VIII (Fig. 141) weakly concave in the middle.

Comparative notes: The pronounced sexual dimorphism of the pronotum, the slender antennae, the similar shapes and chaetotaxy of the male and female sternite VIII, as well as the similar morphology of the aedeagus suggest that A. excisa is closely related to A. desertorum, A. naevula, and allied species, from which it differs by darker average coloration, much more slender antennae, an even more pronounced sexual dimorphism of the pronotum, the shape and chaetotaxy of tergite VIII, and by the shape of the median lobe of the aedeagus. It is readily distinguished from the sympatric and syntopic A. seclusa by the distinctly longer and more slender antennae alone.

Distribution: The known distribution is confined to two localities in western and southern Iran (Map 4). The specimens from the type locality were collected together with A. seclusa at an altitude of $890 \mathrm{~m}$.

\section{Anaulacaspis pamphylica (FAGEL, 1969) \\ (Figs 142-145, Map 5)}

Melagria pamphylica FAGEL, 1969: $21 \mathrm{f}$.

Type material examined: Holotype $\sigma^{\star}$ : "Anatolie mér., Alanya: Dim Irmak, VI.1968. G Fagel / G. Fagel det., pamphylica n.sp. / Type / Anaulacaspis pamphylica (Fagel), det. V. Assing 2016" (IRSNB). Paratypes: 7 exs.: same data as holotype (IRSNB).

Comment: The original description is based on a male holotype and 14 paratypes from "villayet d'Antalya, Alanya, sur plages du Dim Irmak” (FAGEL 1969).

Redescription: Body length 2.2-2.6 mm; length of forebody 1.1-1.2 mm. Coloration: body blackish-brown to black with the posterior margin of the elytra sharply pale-yellow; legs dark-yellowish to pale-brown; antennae dark-brown with the basal two antennomeres paler.

Head distinctly transverse, approximately 1.2 times as broad as long; punctation very fine and sparse; interstices without microsculpture and glossy; sexual dimorphism weakly pronounced. Eyes distinctly longer than postocular region in dorsal view. Antenna 0.8-0.9 mm long; antennomeres IV distinctly oblong, $\mathrm{V}$ approximately as broad as long, VI-X of gradually increasing width and increasingly transverse, $\mathrm{X}$ approximately 1.5 times as broad as long.

Pronotum approximately 1.5 times as broad as long and as broad as head, with pronounced sexual dimorphism.

Elytra slightly longer than pronotum; punctation fine and dense. Hind wings fully developed.

Abdomen narrower than elytra; punctation dense and distinct; posterior margin of tergite VIII (Fig. 142) weakly convex in the middle and with a row of unmodified marginal setae.

$\sigma^{\star}$ : head depressed or (mostly) shallowly impressed in postero-median portion; pronotum with extensive and rather deep median impression and with distinct, sometimes somewhat granulose punctation; sternite VIII 
(Fig. 143) with evenly convex posterior margin; median lobe of aedeagus (Figs 144-145) approximately $0.38 \mathrm{~mm}$ long, with pronounced, long and distinctly concave crista apicalis (lateral view).

\%: head not depressed or impressed in postero-median portion; pronotum without median impression and with extremely fine punctation.

Comparative notes: This species is readily distinguished from the species allied to A. nigra by the sharply yellow posterior margin of the elytra, the pronounced modifications of the male pronotum, and by the much larger median lobe of the aedeagus with a crista apicalis of distinctive shape.

Distribution: Anaulacaspis pamphylica is currently known only from the type locality in the east of Antalya province, South Anatolia (Map 5)

\section{Anaulacaspis flavomarginata spec. nov. (Figs 146-150, Map 5)}

Type material: Holotype ơ: "Albania [6], Elbasan, 9 km ENE Elbasan, 120 m, Shkumbin river bank, 41 09'37"N, 20¹0'39"E, 24.V.2010, V. Assing / Holotypus ơ Anaulacaspis flavomarginata sp. n., det. V. Assing 2016" (cAss). Paratypes: 4 우 + same data as holotype (cAss); 4 exs.: same data, but leg. Schülke (MNB, cAss); 1 ㅇ: "GR. Fthiotis, No. 14, $40 \mathrm{~km}$ W Lamia, bank of Sperchios river, 3855'46N, 2159'19E, 16.IV.2000, V. Assing” (cAss).

Etymology: The specific epithet (Latin, adjective) alludes to the yellow posterior margin of the elytra.

Description: Body length 2.3-2.5 mm; length of forebody 1.1-1.2 mm. Coloration: body black, except for the bright-yellowish posterior margin of the elytra; legs brown to dark-brown; antennae blackish-brown with the basal two antennomeres more or less distinctly paler.

Head approximately 1.15 times as broad as long, with indistinct sexual dimorphism; punctation moderately dense. Eyes longer than postocular region in dorsal view. Antenna approximately $0.8 \mathrm{~mm}$ long; antennomere IV approximately as long as broad; antennomeres V-X of gradually increasing width and increasingly transverse, $\mathrm{X}$ approximately twice as broad as long.

Pronotum 1.10-1.15 times as broad as long and approximately as broad as head; sexual dimorphism rather pronounced.

Elytra slightly longer than pronotum; punctation very dense and very fine. Hind wings fully developed.

Abdomen approximately as broad as elytra, or nearly so; punctation distinct, rather dense, and more or less regularly distributed; posterior margin of tergite VIII (Fig. 146) weakly convex, postero-laterally without a cluster of dense long setae, marginal setae thin and moderately long. $\sigma^{*}$ : punctation of head slightly more distinct than in female; pronotum with dense and distinct, but not asperate punctation; posterior margin of sternite VIII (Fig. 147) obtusely pointed in the middle, marginal setae moderately long, thin, and evenly spaced; median lobe of aedeagus (Figs 148-149) $0.3 \mathrm{~mm}$ long, with rather stout ventral process.

+ : punctation of pronotum very fine; posterior margin of sternite VIII (Fig. 150) weakly concave in the middle.

Comparative notes: Anaulacaspis flavomarginata is readily distinguished from its sympatric congeners as follows:

from A. nigra by the coloration of the elytra, the nonasperate punctation of the male pronotum, the different shape and chaetotaxy of tergite VIII and of the male and female sternite VIII, as well as by the completely different shape of the median lobe of the aedeagus;

from A. laevigata by the coloration of the elytra, darker legs, the sexual dimorphism of the pronotum, and a larger aedeagus with a stouter and apically less acute ventral process;

from A. pamphylica, with which it shares the coloration of the elytra, by a slightly smaller and more slender body, the absence of distinct impressions on the male head and pronotum, as well as by a much smaller aedaegus with a ventral process and crista apicalis of different shapes.

Based on the morphology of the aedeagus, the modifications of the male pronotum, the shapes of tergite and sternite VIII, and particularly the general morphology of the median lobe of the aedeagus, A. flavomarginata is closely related to $A$. desertorum, A. seclusa, and allied species, from which it differs by the coloration and the aedeagus with a ventral process of different shape (lateral view).

Distribution and natural history: The currently known distribution is confined to two localities in Albania and Greece (Map 5). The specimens were hand-collected and washed from gravel on river banks at altitudes of 120 and $280 \mathrm{~m}$, respectively.

\section{Anaulacaspis laevigata (DuviviER, 1883) \\ (Figs 161-158, Map 4)}

Falagria laeviuscula EpPELSHeim, 1880: 204; preoccupied. Falagria laevigata Duvivier, 1883: 94.

Type material examined: Lectotype $\sigma^{\top}$, present designation: "Dalmatien, Knin, Reitter 79. / 1881-I / laeviuscula Eppelsh. Verh. zool. bot. Ges. Wien 1880. p. 204 / c. Epplsh. Steind, d. / Typus / Lectotypus $\sigma^{*}$ Falagria laeviuscula Eppelsheim, rev. V. Assing 2016 / Anaulacaspis laevigata (Duvivier), det. V. Assing 2016" (NHMW). Paralectotypes: $10^{\star}, 2$ 우 오 same data as lectotype; 1 ex.: "Dalmatia, Spalato b. Knin, 1879. Reitter / coll. Reitter / Holotypus 1880, Falagria laeviuscula 
Eppelsheim [curator label]" (HNHM); 1 ex.: "Dalmatien, Spalato, Reitter 79. / coll. Reitter / Paratypus 1880, Falagria laeviuscula Eppelsheim [curator label]” (HNHM).

Comment: The original description of Falagria laeviuscula is based on an unspecified number of syntypes "in wenigen Exemplaren an den Sümpfen bei Knin in Dalmatien ... aufgefunden" (Eppelsheim 1880). The name is a primary homonym and was subsequently replaced with the nomen novum Falagria laevigata by DUvivieR (1883). Four syntypes, two males and two females, were located in the Eppelsheim collection at the NHMW, two additional syntypes were found in the collection at the HNHM. One of the males from the Eppelsheim collection is designated as the lectotype.

Additional material examined: Italy: 1 , "Roma" (NHMW); 2 ㅇ ㅇ , Roma, Valle Aniene, Tivoli, 27.IV.1958 (cTro); $1 \sigma^{*}$, Lazio, Turania (RI) $\left[42^{\circ} 08^{\prime} \mathrm{N}, 12^{\circ} 59^{\prime} \mathrm{E}\right]$, 15.VI.1977, leg. Ippiliti (cZan).

Croatia: 1 ex., Metkovi, leg. Holdhaus (NHMW); 3 exs., Ludbreg, leg. Apfelbeck (NHMW); 1 ex., Opuzen, 1905, leg. Tolatzek (NHMW); 1 ex., Dubrovnik, Mlini, 23.IX.1958 (MHNG); 5 exs., Mlini, 27.IX.-4.X.1958, leg. Benick (MHNG); 1 ex., locality not specified, leg. Apfelbeck (NHMW).

Montenegro: 11 exs., Herzeg-Novi, leg. Holdhaus, Paganetti (MNB, NHMW, cAss); 2 exs., Savina [monastery; $42^{\circ} 27^{\prime} \mathrm{N}, 18^{\circ} 33^{\prime} \mathrm{E}$ ], leg. Paganetti (MNB); 1 o $^{\top}, 1$ 웅, Morača monastery, stream bank, 20.IX.1973, leg. Puthz (MHNG).

Bosnia-Herzegovina: 2 우, Mostar, bank of Neretva river, 13.VIII.1977 (MHNG); 3 exs., locality not specified (NHMW).

Greece: Corfu: 3 exs., locality not specified, leg. Reitter, Winkler (MHNG, NHMW). Pelopónnisos: $60^{\star} 0^{\star}, 2$ 우, 1 ex., Olympia, river bank, 29.III.1988, leg. Schmidt (cAss); $1 \sigma^{\star}, 4$ exs., Patras env., Miha, $900 \mathrm{~m}$, stream bank, 30.III.1986, leg. Assing (cAss). Lesbos: $10^{\text {t }}, 2$ 우 우, Lambou Mili, 390' $\mathrm{N}, 26^{\circ} 23^{\prime} \mathrm{E}, 110 \mathrm{~m}$, sifted, 19.III.2005, leg. Lompe \& Meybohm (cAss); $10^{*}, 14 \mathrm{~km}$ WNW Mytilini, S Lambou Mili, $39^{\circ} 08^{\prime} \mathrm{N}, 26^{\circ} 24^{\prime} \mathrm{E}, 50 \mathrm{~m}$, stream bank, 22.III.2016, leg. Assing \& Hetzel (cAss).

Turkey: Ordu: $1 o^{\star}, 2$ 우 우 $18 \mathrm{~km}$ SSE Gölköy, 40³2' N, $37^{\circ} 41^{\prime} \mathrm{E}, 970 \mathrm{~m}$, river bank, 14.VII.2008, leg. Schülke (MNB, cAss). Antalya: 4 우, SSW Antalya, Çiralı, $36^{\circ} 25^{\prime} \mathrm{N}, 30^{\circ} 28^{\prime} \mathrm{E}$, floated from stream bank 4.IV.2002, leg. Assing \& Wunderle (cAss, cWun). Adana: 2 우 우, Feke env., 21.-24.VII.2000, leg. Smatana (MNB, cAss). Osmaniye: 1 , $15 \mathrm{~km}$ Osmaniye, NW Yarpuz, $37^{\circ} 04^{\prime} \mathrm{N}$, $36^{\circ} 26^{\prime} \mathrm{E}, 920 \mathrm{~m}$, stream bank, floated from stream bank, leg. Assing \& Schülke (MNB).

Redescription: Body length 1.8-2.2 mm; length of forebody $0.9-1.0 \mathrm{~mm}$. Coloration: head, pronotum, and abdomen blackish-brown to black; elytra dark-brown to black; legs pale-brown to dark-brown; antennae dark-brown to blackish-brown with the basal two antennomeres usually slightly paler.

Head approximately 1.1 times as broad as long, without sexual dimorphism; punctation extremely fine, visible only at high magnification $(50 \mathrm{x})$, and moderately dense; interstices glossy. Eyes approximately as long as postocular region in dorsal view, or slightly longer. Antenna 0.6-0.7 mm long; antennomere IV approximately as long as broad or weakly oblong; antennomeres $\mathrm{V}-\mathrm{X}$ of gradually increasing width and increasingly transverse, $\mathrm{X}$ approximately twice as broad as long, or nearly so.

Pronotum approximately 1.05 times as broad as long and approximately 0.95 times as broad as head, without sexual dimorphism; punctation very fine, sometimes slightly more distinct than that of head.

Elytra usually $0.90-0.95$ times as long as pronotum; punctation less dense and slightly less fine than that of pronotum. Hind wings present.

Abdomen approximately as broad as, or slightly broader than elytra; punctation distinct, denser in anterior than in posterior portions of tergites; posterior margin of tergite VIII (Fig. 151) weakly convex, postero-laterally without a cluster of dense long setae, marginal setae unmodified and moderately long.

$0^{*}$ : posterior margin of sternite VIII (Fig. 152) strongly convex or obtusely pointed in the middle, marginal setae moderately long, thin, and evenly spaced; median lobe of aedeagus $0.25-0.28 \mathrm{~mm}$ long and shaped as in Figs 153-157.

ㅇ: posterior margin of sternite VIII weakly concave in the middle (Fig. 158).

Comparative notes: Anaulacaspis laevigata is distinguished from the species of the A. nigra group by the absence of postero-lateral clusters of long thin setae on tergite VIII, a posteriorly less produced male sternite VIII, the shape of the female sternite VIII (posteriorly less strongly convex and in the middle concave), and the less slender median lobe of the aedeagus, from nearly all the representatives of the A. nigra group also by the extremely fine punctation of the non-dimorphic head and pronotum. For characters separating it from the similar A. iberica see the comparative notes in the following section.

Distribution and natural history: Confirmed records of this rare species are known from South Italy, the Balkans, and Turkey (Map 4). Thus, the distribution is evidently of the Ponto-Mediterranean type sensu LatTin (1967). According to Schülke \& Smetana (2015), A. laevigata has also been recorded from Uzbekistan, but this record most likely refers to A. nigerrima. Horion (1967) reports three records from southern Austria (Kärnten, Steiermark) communicated to him by Otto Scheerpeltz. However, there is no reference material whatsoever in the collections of the NHMW, so that these records are considered erroneous. 
Most of the recently collected, revised specimens were collected on the banks of rivers and streams, usually from gravel, whereas HoRIon (1967) states that A. laevigata lives in swamps.

\section{Anaulacaspis iberica spec. nov.} (Figs 159-166, Map 4)

Type material: Holotype ơ: "Belus [= Beloso; 42 $19^{\prime} \mathrm{N}$, $8^{\circ} 45^{\prime} \mathrm{W}$ ], lg. H. Franz / Sp. 184 [overleaf] / Prov. Pontevedra, Hispania bor. / Holotypus ơ Anaulacaspis iberica sp. n., det. V. Assing 2016" (NHMW). Paratypes: 4 exs.: same data as holotype (NHMW, cAss); 2 exs: "Sobradelo [ $\left.42^{\circ} 24^{\prime} \mathrm{N}, 6^{\circ} 54^{\prime} \mathrm{W}\right]$, Valle de Casayo, lg. H. Franz / Sp 202 [overleaf]" (NHMW); 1 \%: "Prov. Jaen, Sierra Cazorla, Besuchet 12.IV.59 / G. Fagel det., 1960, Falagria nigra Grav." (MHNG).

Etymology: The specific epithet (Latin, adjective) alludes to the fact that this species is currently known only from the Iberian Peninsula.

Description: Body length 1.6-2.2 mm; length of forebody $0.8-1.0 \mathrm{~mm}$. Coloration: head and abdomen blackishbrown; pronotum and elytra brown to blackish-brown; legs pale-brown to brown; antennae dark-brown to blackishbrown with the basal antennomeres rarely paler.

Head approximately 1.1 times as broad as long, without sexual dimorphism; punctation extremely fine, visible only at high magnification $(50 \mathrm{x})$, and rather dense; interstices glossy. Eyes approximately as long as postocular region in dorsal view. Antenna $0.6-0.7 \mathrm{~mm}$ long; antennomere IV approximately as long as broad; antennomeres $\mathrm{V}-\mathrm{X}$ of gradually increasing width and increasingly transverse, $\mathrm{X}$ approximately twice as broad as long.

Pronotum approximately 1.1 times as broad as long and approximately as broad as head, without sexual dimorphism; punctation very fine, but slightly more distinct than that of head.

Elytra approximately 0.85 times as long as pronotum; punctation less dense and less fine than that of pronotum. Hind wings not examined, possibly of reduced length.

Abdomen slightly broader than elytra; punctation distinct, denser in anterior than in posterior portions of tergites; posterior margin of tergite VIII (Fig. 164) weakly convex, postero-laterally without a cluster of dense long setae, marginal setae unmodified and moderately long.

$\sigma^{\top}$ : posterior margin of sternite VIII (Fig. 165) obtusely pointed in the middle, marginal setae moderately long, thin, and evenly spaced; median lobe of aedeagus $0.23-$ $0.24 \mathrm{~mm}$ long and shaped as in Figs 159-163.

$\uparrow:$ posterior margin of sternite VIII (Fig. 166) distinctly concave in the middle.

Comparative notes: Aside from the (on average) slightly larger and more transverse pronotum and the (on average) shorter elytra, the external characters of A. iberica are practically identical to those of A. laevigata, evidently its adelphotaxon. The new species is reliably distinguished from A. laevigata only by the slightly smaller median lobe of the aedeagus with a more slender (lateral view) and relatively longer (in relation to capsule) ventral process and with a smaller and more prominent crista apicalis, and by the more distinctly concave middle of the posterior margin of the female sternite VIII.

Distribution: The known distribution is confined to two localities in Galicia, northwestern Spain, and one in Andalucía, South Spain (Map 4). Previous records of A. nigra from Spain and Portugal probably refer to this species.

\section{Anaulacaspis nigrina (FAGEL, 1969) \\ (Figs 167-172, Map 3)}

Melagria nigrina FAGEL, 1969: $13 \mathrm{ff}$.

Type material examined: Holotype ${ }^{\text {T: }}$ "Anatolie mér., Alanya: Dim Irmak, VI.1968 G. Fagel / G. Fagel det., nigrina n. sp. / Type / Anaulacaspis nigrina (Fagel), det. V. Assing 2016" (IRSNB). Paratypes: 22 exs.: same data as holotype (IRSNB).

Comment: The original description is based on a male holotype and "Nombreux exemplaires" from "Alanya ... Dim Irmak” (FAGEL 1969).

Additional material examined: Greece: Lesbos: $1 \sigma^{\top}, 1$ 운, Lambou Mili, $39^{\circ} 08^{\prime} \mathrm{N}, 26^{\circ} 23^{\prime} \mathrm{E}, 110 \mathrm{~m}$, sifted, 19.III. 2005 , leg. Lompe \& Meybohm (cAss); $2 \sigma^{\top} \sigma^{7}, 14 \mathrm{~km}$ WNW Mytilini, S Lambou Mili, $39^{\circ} 08^{\prime} \mathrm{N}, 26^{\circ} 24^{\prime} \mathrm{E}, 50 \mathrm{~m}$, stream bank, 22.III.2016, leg. Assing \& Hetzel (cAss); $10^{\text {Tे, same }}$ data, but 28.III.2016 (cAss)

Turkey: Muğla: $1 o^{7}, 6+9+25 \mathrm{~km}$ E Fethiye, Kemer, $36^{\circ} 38^{\prime} \mathrm{N}, 29^{\circ} 22^{\prime} \mathrm{E}$, river bank, 4.X.2002, leg. Assing (cAss). Isparta: 1 \&, $10 \mathrm{~km} \mathrm{SE} \mathrm{Sütçüler,} 37^{\circ} 25^{\prime} \mathrm{N}, 31^{\circ} 02^{\prime} \mathrm{E}, 1520 \mathrm{~m}$, meadow, under stones, 26.IV.2011, leg. Assing (cAss). Antalya: $10^{\star}, 32 \mathrm{~km}$ NE Mahmutlar, N Karapınar, Gevne Cay river, $36^{\circ} 37^{\prime} \mathrm{N}, 32^{\circ} 25^{\prime} \mathrm{E}, 1050 \mathrm{~m}, 29 . \mathrm{V} .2006$, leg. Skale (cAss); 1 ㅇ, Beskonak, 31.V.1992, leg. Gillerfors (cWun). Kahramanmaras: 1 i , $60 \mathrm{~km}$ W Kahramanmaras, N Andirm, $37^{\circ} 37^{\prime} \mathrm{N}, 36^{\circ} 21^{\prime} \mathrm{E}, 1120 \mathrm{~m}$, stream bank, flood debris, 26.III.2005, leg. Assing (cAss).

Iran: Kordestan: $10^{7}, 27 \mathrm{~km}$ SW Saqqez, $2 \mathrm{~km}$ SW Mir Deh, $36^{\circ} 08^{\prime} \mathrm{N}, 46^{\circ} 03^{\prime} \mathrm{E}, 1600 \mathrm{~m}$, 3.IX.2008, leg. Frisch \& Serri (MNB). Ilam: 3 ㅇ ㅇ, $10 \mathrm{~km}$ NW Eyvan, $5 \mathrm{~km}$

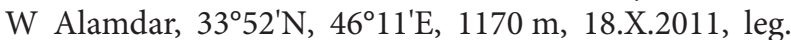
Frisch (MNB, cAss).

Redescription: Body length 1.7-2.2 mm; length of forebody $0.8-1.1 \mathrm{~mm}$. Coloration: whole body including appendages black, with parts of the tibiae and tarsi sometimes and the narrow posterior margins of the elytra rarely indistinctly paler dark-brown to blackish-brown. 
Head approximately 1.15 times as broad as long, with weakly pronounced sexual dimorphism. Eyes slightly longer than postocular region in dorsal view. Antenna 0.6-0.7 mm long.

Pronotum approximately 1.15 times as broad as long and as broad as head, with moderately pronounced sexual dimorphism.

Elytra approximately as long as pronotum; punctation fine and dense; interstices without microsculpture. Hind wings fully developed.

Abdomen approximately as broad as, or slightly narrower than, elytra; punctation dense and distinct; interstices without appreciable microsculpture; tergite VIII (Fig. 167) with weakly concave posterior margin and with long and unmodified marginal setae, postero-laterally without cluster of long thin setae.

$\sigma^{*}$ : head with or without indistinct depression in posteromedian portion and with fine, distinct, and moderately dense punctation; pronotum shallowly and usually extensively depressed or impressed in the middle, punctation distinct and moderately dense, not asperate; sternite VIII (Fig. 168) strongly convex posteriorly; median lobe of aedeagus (Figs 169-171) approximately $0.25 \mathrm{~mm}$ long, ventral process apically acute in lateral view, slender in ventral view.

i : head with fine punctation, on average somewhat finer than in male; pronotum with the usual small posteromedian impression near posterior margin, but without extensive impression in postero-median portion, punctation finer than in male; sternite VIII (Fig. 172) strongly transverse, posterior margin broadly concave, in the middle weakly concave.

Comparative notes: Despite some external resemblance to some species of the A. nigra group, A. nigrina is more closely related to A. laevigata and allied species, as can be inferred from the absence of a postero-lateral cluster on tergite VIII, from the shapes of the male and female sternites VIII, and from the morphology of the median lobe of the aedeagus. It is distinguished from the similarly coloured and similarly finely punctate A. nigerrima and $A$. libanotica of the $A$. nigra group by the chaetotaxy of tergite VIII (without a postero-lateral cluster of long setae on either side), by the completely different shapes of the male and female sternites VIII, by smaller body size, and by the morphology of the median lobe of the aedeagus.

Distribution and natural history: Based on the examined material, the distribution ranges from the Greek island Lesbos across Turkey eastwards to Iran (Map 3). The examined material was collected on the banks of rivers and streams either by hand-collecting or by sifting debris; one specimen was found under a stone in a meadow. The altitudes range from 50 to $1600 \mathrm{~m}$.

\section{Anaulacaspis beijingensis (PACE, 1998) \\ (Figs 173-177, Map 7)}

Melagria beijingensis PACE, 1998: 398.

Melagria marginata PACE, 1998: 400; syn. n.

Type material examined: $M$. beijingensis: Holotype $\sigma^{\top}$ : "CHINA Beijing, B.N.U at light, V-VI.1993, G. de Rougemont / Holotypus Melagria beijingensis, det. R. Pace 1995 / Melagria beijingensis sp. n., det. R. Pace 1995 / Anaulacaspis beijingensis (Pace), det. V. Assing 2016” (MHNG). M. marginata: Holotype ㅇ: "CHINA Beijing, Panshan, 8.v.1995, G. de Rougemont / Holotypus Melagria marginata, det. R. Pace 95 / Melagria marginata sp. n., det. R. Pace 1995 / Anaulacaspis beijingensis (Pace), det. V. Assing 2016" (MHNG).

Comment: The original description of A. beijingensis is based on a male holotype from "China, Beijing, B.N.U., at light" and two female paratypes from "China Yingtaogou" (PACE 1998), that of M. marginata on a unique female holotype from "China, Beijing, Panshan" (PACE 1998). According to PACE (1998), A. marginata is distinguished from $A$. beijingensis by completely brown antennae (A. beijingensis: antennomeres I-II yellowishbrown), distinctly transverse antennomeres IX and X, parallel temples, a distinctly transverse pronotum, the coloration of the elytra (yellowish coloration extending anteriad along suture), and by the less strongly sclerotized and shorter distal portion of the spermathecal capsule. An examination of the holotypes of $A$. beijingensis and A. marginata, as well as of additional material from Shaanxi, whose aedeagus is identical to that illustrated by PACE (1998) for A. beijingensis, revealed pronounced intraspecific variation regarding all these characters, but no significant differences. Consequently, there is little doubt that the holotype of A. marginata is conspecific with that of $A$. beijingensis. Both names were made available in the same article. Anaulacaspis beijingensis is designated as the senior synonym, since this name is represented by a male type specimen.

Additional material examined: China: Shaanxi: $7 \sigma^{\top} \sigma^{*}$, 4 우 ㅇ, $15 \mathrm{~km} \mathrm{~N}$ Xi'an, road to Xi'an airport, bank of Wei $\mathrm{He}$ river, $34^{\circ} 24^{\prime} \mathrm{N}, 108^{\circ} 55^{\prime} \mathrm{E}, 400 \mathrm{~m}$, 22.VIII.1995, leg. Wrase \& Schülke (MNB, cAss).

Redescription: Body length 1.8-2.2 mm; length of forebody $0.9-1.0 \mathrm{~mm}$. Coloration: head, pronotum, and abdomen blackish-brown to blackish; elytra dark-brown to blackish-brown, posterior margin and sometimes also the sutural portion more or less extensively yellowish; legs dark-yellowish to yellowish-brown; antennae dark-brown to blackish brown, with the basal 2-3 antennomeres often more or less distinctly paler.

Head approximately 1.15 times as broad as long, with more or less pronounced sexual dimorphism; punctation fine and dense in both sexes. Eyes longer than postocular 
region in dorsal view. Antenna $0.7-0.8 \mathrm{~mm}$ long and with sexual dimorphism.

Pronotum 1.10-1.15 times as broad as long and approximately as broad as head, with pronounced sexual dimorphism.

Elytra approximately as long as, or slightly shorter than, pronotum; punctation dense and fine. Hind wings present.

Abdomen approximately as broad as elytra; punctation dense and distinct; interstices without appreciable microsculpture; tergite VIII (Fig. 173) strongly transverse and with truncate posterior margin, marginal setae moderately long and unmodified.

$0^{*}$ : head flattened or more or less extensively and more or less distinctly impressed; antenna approximately $0.8 \mathrm{~mm}$ long, antennomeres IV weakly oblong, $\mathrm{V}$ approximately as broad as long, and VI-X of gradually increasing width and increasingly transverse, $\mathrm{X}$ approximately 1.5 times as broad as long; pronotum with extensive median impression and with dense and distinct punctation; sternite VIII (Fig. 174) with strongly convex posterior margin, marginal setae fine and rather short; median lobe of aedeagus (Figs 175-176) approximately $0.33 \mathrm{~mm}$ long; ventral process apically very acute both in lateral and in ventral view.

i : head neither flattened nor impressed; antenna approximately $0.7 \mathrm{~mm}$ long, antennomeres IV approximately as broad as long, $\mathrm{V}-\mathrm{X}$ of gradually increasing width and increasingly transverse (more so than in male), X nearly twice as broad as long; pronotum without distinct median impression and with fine punctation; sternite VIII (Fig. 177) strongly transverse, posterior margin broadly concave in the middle and with moderately long and fine marginal setae.

Comparative notes: Based on the morphology of the aedeagus, the pronounced sexual dimorphism of the pronotum, as well as on the shapes of tergite VIII and sternite VIII, A. beijingensis belongs to the A. naevula group. Among the species of this group it is characterized particularly by the sexual dimorphism of the antennae (unique), the coloration, the shape and chaetotaxy of tergite VIII, and the conspicuously acute apex of the ventral process of the aedeagus.

Distribution and natural history: Anaulacaspsis beijingensis has been recorded only from Beijing and Shaanxi provinces, China (Map 7). The additional material was collected on a river bank at an altitude of $400 \mathrm{~m}$.

\section{Anaulacaspis schuelkei (Assing, 2001)}

(Map 7)

Cordalia schuelkei Assing, 2001: $116 \mathrm{ff}$.

Comment: The original description is based on a unique holotype from "the Qin Ling Shan in Shaanxi (China)"
(Assing 2001). The species was moved to Anaulacaspis by Assing (2009). For a detailed description and illustrations of tergite VIII and the male primary and secondary sexual characters see Assing (2001). Based on the chaetotaxy of tergite VIII (marginal setae unmodified; postero-laterally without a cluster of long setae on either side) and on the morphology of the aedeagus, the species is tentatively assigned to the A. naevula group.

Distribution: This species is currently known only from the type locality in the Qinling Shan in Shaanxi (Map 7).

\section{Anaulacaspis taiwanica PACE, 2008 \\ (Figs 178-183, Map 7)}

Anaulacaspis taiwanica PACE, 2008: 255

Type material examined: Paratypes: 10 , 1 : : "TAIWAN, Taitung Hsien, Foothills of Hsinkangshan nr. Chengkung, 350-400 m, 19.VII.93, A. Smetana [T155] / Paratypus Melagria [sic] taiwanica mihi, det. R. Pace, 2005 / Melagria [sic] taiwanica mihi, det. R. Pace, 2005 / Anaulacaspis taiwanica Pace, det. V. Assing 2016” (NSMT).

Comment: The original description is based on seven specimens collected in two localities in Hsinkangshan (near Chengkung), Taitung Hsien, Taiwan (PAcE 2008).

Redescription: Body length $2.2-2.3 \mathrm{~mm}$; length of forebody $0.95-1.05 \mathrm{~mm}$. Coloration: head and abdomen black; pronotum and elytra blackish-brown, with the postero-sutural portion slightly and diffusely paler; legs yellowish; antennae dark-brown to blackish-brown, with the basal 2-3 antennomeres somewhat paler. Forebody without microsculpture and glossy.

Head transverse, 1.10-1.15 times as broad as long, without sexual dimorphism; punctation extremely fine and moderately sparse. Eyes longer than postocular region in dorsal view. Antenna $0.7-0.8 \mathrm{~mm}$ long; antennomeres IV weakly oblong, V approximately as broad as long, and VI-X increasingly transverse and of gradually increasing width, $\mathrm{X}$ approximately twice as broad as long.

Pronotum approximately 1.15 times as broad as long and as broad as head, with moderately pronounced sexual dimorphism; punctation dense and very fine, but more distinct than that of head.

Elytra approximately as long as pronotum; punctation dense and very fine. Hind wings fully developed.

Abdomen slightly narrower than elytra; tergites IIIIV with deep anterior impressions, tergite $\mathrm{V}$ without distinct anterior impression; tergites III-VI with dense and distinct, tergite VII with fine and sparse punctation; tergite VIII (Figs 178, 182) with moderate sexual dimorphism, anteriorly with distinct median excision, without postero-lateral clusters of setae.

$\sigma^{*}$ : pronotum with shallow, but extensive median impression in posterior two-thirds; posterior margin of 
tergite VIII (Fig. 178) broadly concave and with a row of long and dense marginal setae, this row broadly interrupted in the middle; sternite VIII (Fig. 179) broadly and convexly produced posteriorly; median lobe of aedeagus (Figs 180-181) rather large in relation to body size, approximately $0.4 \mathrm{~mm}$ long; ventral process apically curved in lateral view, basally with subparallel lateral margins in ventral view.

\%: pronotum with the usual posterior impression, otherwise unmodified; posterior margin of tergite VIII (Fig. 182) truncate, marginal setae moderately dense and moderately long, present also in the middle of posterior margin; posterior margin of sternite VIII (Fig. 183) weakly concave in the middle.

Comparative notes: Among East Palaearctic Anaulacaspis species of similar body size, coloration, and fine punctation, A. taiwanica is characterized by the modifications of the male pronotum, the sexually dimorphic shape and chaetotaxy of tergite VIII, and particularly by the shape of the median lobe of the aedeagus. Based on the chaetotaxy of tergite VIII (marginal setae unmodified; postero-laterally without a cluster of long setae on either side) and on the morphology of the aedeagus, the species is tentatively assigned to the A. naevula group.

Distribution: The currently known distribution is confined to two close localities in Taitung Hsien, Taiwan (Map 7).

\section{Anaulacaspis gilva spec. nov.}

(Figs 184-190, Map 6)

Type material: Holotype $0^{\star}$ : "PAK, Northern Areas, Ghizar valley, Thiee, $36.0229^{\circ} \mathrm{N}, 74.1681^{\circ} \mathrm{E}$, 5.7.2007 $1760 \mathrm{~m}$, leg. H. Mühle / Holotypus ơ Anaulacaspis gilva sp. n., det. V. Assing 2016" (MNB). Paratypes: $20^{\star} o^{\star}$, 2 ㅇ ㅇ: same data as holotype (MNB, cAss); 1 우 "PAK, Northern Areas, Chilas LF, $35.4310^{\circ} \mathrm{N}, 74.1002^{\circ} \mathrm{E}$, 7.7.2007 $1075 \mathrm{~m}$, leg. H. Mühle" (MNB); 1 \%: “PAK, Northern Areas, $15 \mathrm{~km}$ E Chilas, $35.4215^{\circ} \mathrm{N}, 74.2711^{\circ} \mathrm{E}$, 7.7.2007 1100 m, leg. H. Mühle" (MNB).

Etymology: The specific epithet (Latin, adjective: yellow) alludes to the pale coloration of this species.

Description: Body length 1.8-2.1 mm; length of forebody $0.95-1.05 \mathrm{~mm}$. Coloration: head dark-yellowish to yellowish-red; pronotum reddish-yellow; elytra yellow with a very indistinct, pale, small, and weakly delimited medio-lateral infuscate spot; abdomen yellow, usually with the antero-median portion of tergite VI black, rarely with the median portion of tergite VI more extensively and also the median portion of tergite $\mathrm{V}$ infuscate; legs pale-yellowish; antennae brown with the basal 3-4 antennomeres pale-yellowish.

Head approximately 1.1 times as broad as long, without sexual dimorphism; punctation sparse and extremely fine. Eyes longer than postocular region in dorsal view. Antenna $0.7-0.8 \mathrm{~mm}$ long and distinctly incrassate apically; antennomere IV weakly oblong; antennomeres VI-X of gradually increasing width and increasingly transverse, IX-X more than 1.5 times as broad as long.

Pronotum approximately 1.1 times as broad as long and as broad as head, or nearly so, without sexual dimorphism; with a pronounced, but small median impression near posterior margin, this impression anteriorly sometimes extending into short and fine sulcus; punctation very fine and dense, slightly more distinct and denser than that of head.

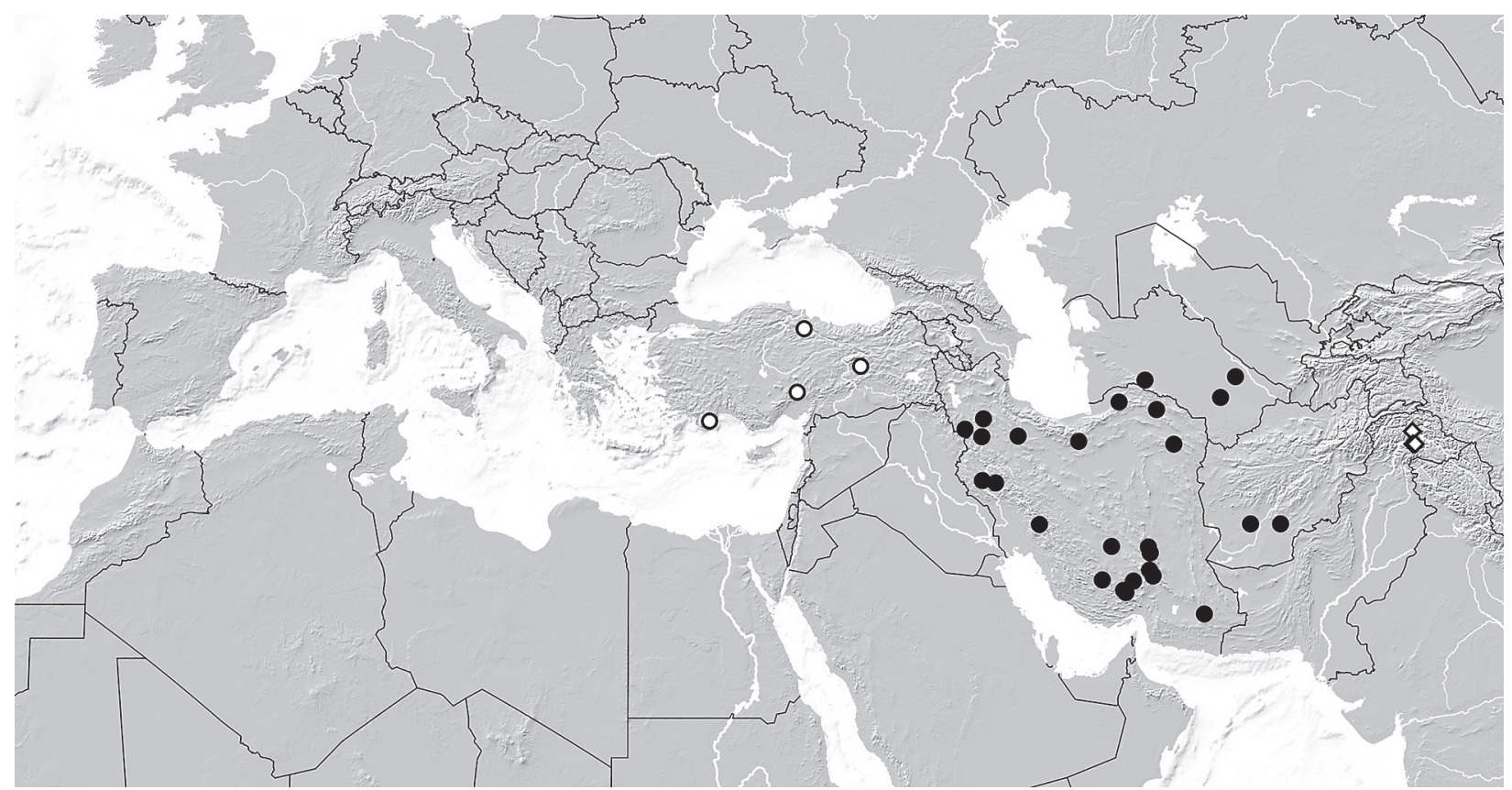

Map 6: Distributions of Anaulacaspis convexa (white circles), A. seclusa (black circles), and A. gilva (white diamonds) in the West Palaearctic and Middle Asia, based on revised records. 
Elytra approximately 0.9 times as long as pronotum; punctation very fine and rather sparse. Hind wings fully developed.

Abdomen approximately as broad as elytra; punctation very fine and moderately dense, anterior and posterior portions of tergites with sparse punctation; tergite VIII (Figs 184, 189) with truncate posterior margin and with a comb-like row of slightly modified marginal setae.

$\mathrm{o}^{\text {t: }}$ : posterior margin of sternite VIII (Fig. 185) obtusely pointed in the middle, marginal setae thin and not very long; median lobe of aedeagus (Figs 187-188) approximately $0.35 \mathrm{~mm}$ long and slender; ventral process very slender and apically acute both in lateral and in ventral view; internal sac with a long dark structure.

o : posterior margin of sternite VIII (Fig. 190) broadly and shallowly concave in the middle; spermatheca small and comma-shaped, not distinctive.

Comparative notes: As can be inferred from the similarly derived chaetotaxy of tergite VIII and from the similarly derived morphology of the aedeagus (ventral process very long and slender both in lateral and in ventral view; internal sac with a long dark structure), A. gilva is undoubtedly most closely related to A.pectinata (see the following section). It is distinguished from this species by smaller size, paler coloration, less slender and shorter antennae with more strongly transverse antennomeres $\mathrm{V}-\mathrm{X}$, smaller and less bulging eyes, shorter and less broad elytra, a truncate posterior margin of tergite VIII with shorter and less dense marginal setae, and a slightly smaller aedeagus with a ventral process of slightly different shape.
Distribution: The known distribution is confined to North Pakistan (Map 6). The specimens were collected at altitudes of 1075-1760 m, one of them with a light trap.

\section{Anaulacaspis pectinata spec. nov.}

(Figs 191-195, Map 7)

Type material: Holotype ơ: "CHINA: Yunnan, Baoshan Pref., creek valley $21 \mathrm{~km} \mathrm{~S}$ Tengchong, $1358 \mathrm{~m}$, $24^{\circ} 50^{\prime} 22^{\prime \prime} \mathrm{N}, 98^{\circ} 27^{\prime} 01^{\prime \prime} \mathrm{E}$, washed from creek border and gravel bank, 30.VIII.2009, leg. M. Schülke [CH09-20] / Holotypus ơ Anaulacaspis pectinata sp. n., det. V. Assing 2016" (MNB).

Etymology: The specific epithet is an adjective derived from the Latin noun pecten (comb) and alludes to the comb-like setae at the posterior margin of tergite VIII.

Description: Body length $2.4 \mathrm{~mm}$; length of forebody $1.3 \mathrm{~mm}$. Coloration: head dark reddish-brown; pronotum reddish; elytra pale-reddish with a very indistinct and diffuse medio-lateral infuscate spot; abdomen darkyellowish, with the median portions of tergite V and VII infuscate; legs yellowish; antennae blackish-brown with the basal three antennomeres yellowish.

Head 1.07 times as broad as long, without sexual dimorphism; punctation rather sparse and extremely fine. Eyes much longer than postocular region in dorsal view. Antenna $0.95 \mathrm{~mm}$ long and slender; antennomeres IV and V weakly oblong, VI approximately as broad as long,

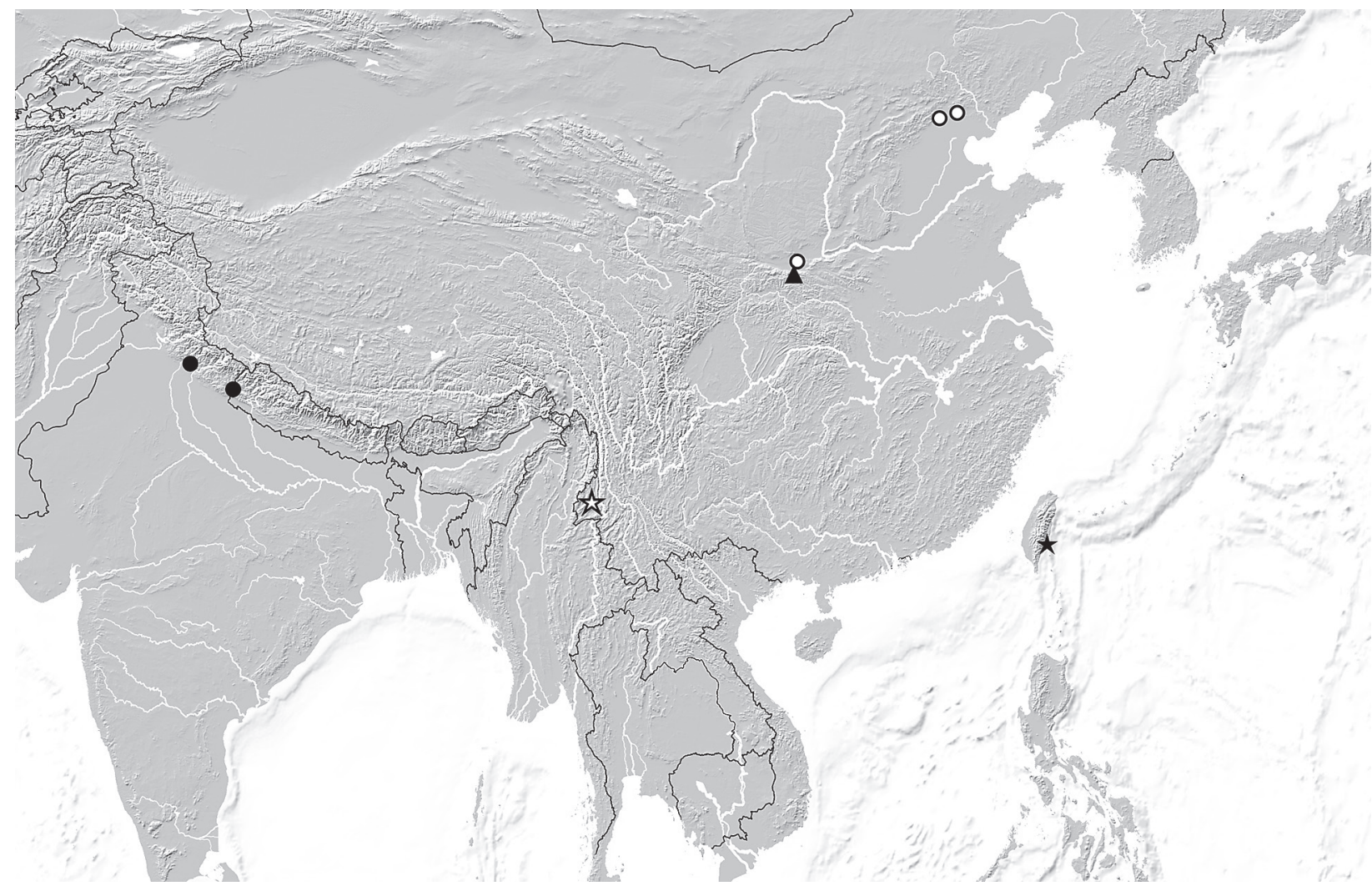

Map 7: Distributions of Anaulacaspis beesoni (black circles), A. pectinata (white star), A. schuelkei (black triangle), A. beijingensis (white circles), and A. taiwanica (black star) in the East Palaearctic region, based on revised records. 
VII-X of gradually increasing width and increasingly transverse, $\mathrm{X}$ barely 1.5 times as broad as long.

Pronotum approximately 1.1 times as broad as long and slightly broader than head, with a pronounced, but small impression near posterior margin, this impression anteriorly extending into short and fine sulcus not reaching middle of pronotum; punctation very fine and dense, more distinct and denser than that of head.

Elytra slightly longer than pronotum; punctation very fine and moderately dense. Hind wings fully developed. Abdomen narrower than elytra; punctation moderately fine and moderately dense, anterior and posterior portions of tergites with sparse punctation; tergite VIII (Figs 191-192) with weakly convex posterior margin and with a comb-like row of slightly modified marginal setae.

$\mathrm{o}^{\star}$ : posterior margin of sternite VIII (Fig. 193) obtusely pointed in the middle, marginal setae thin and not very long; median lobe of aedeagus (Figs 194-195) approximately $0.4 \mathrm{~mm}$ long and slender; ventral process very slender and apically acute both in lateral and in ventral view; internal sac with a long dark structure.

क: unknown.

Comparative notes: For characters distinguishing A. pectinata from its adelphotaxon A. gilva see the comparative notes in the preceding section.

Distribution and natural history: The type locality is situated in Northwest Yunnan at an altitude of approximately $1360 \mathrm{~m}$. The holotype was washed from the gravel bank of a stream.

\section{Anaulacaspis formosa (RoSENHAUER, 1856)}

(Figs 196-211, Map 3)

Falagria formosa RosenHAUER, 1856: $88 \mathrm{f}$.

Falagria cirrosa FAUveL, 1902: 184 f.; syn. n.

Melagria formosa algirica FAgeL, 1969: 30; syn. n.

Melagria formosa elkantarensis FAGEL, 1969: 31 f.; syn. n.

Melagria formosa macra FAGEL, 1969: 30 f.; syn. n.

Melagria formosa biskrensis FAGEL, 1969: 32 ff.; syn. n.

Melagria cirrosa andalusiaca FAGEL, 1969: 36; syn. n.

Type material examined: Falagria formosa: Lectotype \%: "Malaga / Andalusie / Algeciras / Puerto de Gajnrés [?] (Espagne) / formosa Rosh. type / R.I.Sc.N.B. 17.479, Falagria naevula Er., Coll. et det. A. Fauvel / Ex-Typis / G. Fagel elig., 1968, Falagria formosa Rosh., Lectotype / Anaulacaspis formosa (Rosenhauer), det. V. Assing 2016" (IRSNB).

Falagria cirrosa: Lectotype $\sigma^{*}$ [dissected by R. Pace]: “Teboursouk / cirrosa Fvl. / R.I.Sc.N.B. 17.479, Falagria, Coll. et det. A. Fauvel / Ex-Typis / G. Fagel elig., 1968, Falagria cirrosa Fauvel, Lectotype / vidit R. Pace 1985" (IRSNB). Paralectotypes: 1 ㅇ: “Teboursouk 7 / R.I.Sc.N.B. 17.479, Falagria cirrosa Fauv. Coll. et det. A. Fauvel
/ Ex-Typis / Falagria cirrosa Fvl., det. R. Pace 1985" (IRSNB); 1 ㅇ: "Palestro, Algerie / R.I.Sc.N.B. 17.479, Falagria cirrosa Fauv. Coll. et det. A. Fauvel / Ex-Typis / Falagria cirrosa Fvl., det. R. Pace 1985” (IRSNB); 1 \% : "Chabounia, Prov. d'Alger / R.I.Sc.N.B. 17.479, Falagria cirrosa Fauv. Coll. et det. A. Fauvel / Ex-Typis / Falagria cirrosa Fvl., det. R. Pace 1985” (IRSNB); 1 ㅇ [dissected by R. Pace]: "Aïn Draham, 7 Tunisie / R.I.Sc.N.B. 17.479, Falagria cirrosa Fauv. Coll. et det. A. Fauvel / Ex-Typis / Falagria cirrosa Fvl., det. R. Pace 1985" (IRSNB); 1 ㅇ [dissected by R. Pace]: "Bou Berak près Dellys / R.I.Sc.N.B. 17.479, Falagria cirrosa Fauv. Coll. et det. A. Fauvel / Ex-Typis / Falagria cirrosa Fvl., det. R. Pace

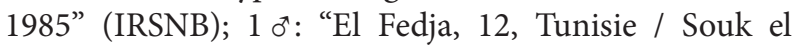
Arba, inond. Madjerda, 1 / R.I.Sc.N.B. 17.479, Falagria cirrosa Fauv. Coll. et det. A. Fauvel / Ex-Typis / Falagria cirrosa Fvl., det. R. Pace 1985” (IRSNB); $10^{\star}$ [dissected by R. Pace], 2 우 우: "Les Lacs, Prov. de Const., Algérie / R.I.Sc.N.B. 17.479, Falagria cirrosa Fauv. Coll. et det. A. Fauvel / Ex-Typis / Falagria cirrosa Fvl., det. R. Pace 1985” (IRSNB); 1 o: “Tunis 9 / R.I.Sc.N.B. 17.479, Falagria cirrosa Fauv. Coll. et det. A. Fauvel / Ex-Typis / Falagria cirrosa Fvl., det. R. Pace 1985” (IRSNB); 2 우 우: "Fernana 8, Tunisie / R.I.Sc.N.B. 17.479, Falagria cirrosa Fauv. Coll. et det. A. Fauvel / Ex-Typis / Falagria cirrosa Fvl., det. R. Pace 1985” (IRSNB).

Melagria formosa algirica: Holotype $0^{*}$ : "Philippeville, Algérie, A. Thery / G. Fagel det., formosa algirica nov. / Type / Anaulacaspis formosa (Rosenhauer), det. V. Assing 2016" (IRSNB). Paratypes: $1 \mathrm{o}^{\text {t}}$ : same data as holotype (IRSNB); 1 ㅇ: "Fil-Fila, Algérie, A. Thery" (IRSNB); 1 운 "Bône / R.I.Sc.N.B. 17.479, Falagria cirrosa Fauv. Coll. et det. A. Fauvel / Ex-Typis / Anaulacaspis cirrosa (Fauvel), det. V. Assing" (IRSNB).

Melagria formosa elkantarensis: Holotype $\sigma^{*}$ [slightly teneral]: "Algérie: Djebel Metlilli, gorges d'El Kantara, V-1954, G. Fagel / formosa elkantarensis nov. / Type / Anaulacaspis formosa (Rosenhauer), det. V. Assing 2016" (IRSNB). Paratypes: $5 \sigma^{\star} \sigma^{\star}, 1$ [partly teneral]: same data as holotype (IRSNB).

Melagria formosa macra: Holotype $\sigma^{\star}$ : "Algérie: Algerois, Kaddous, 3-v-1954, G. Fagel / formosa macra nov. / Type / Anaulacaspis formosa (Rosenhauer), det. V. Assing 2016" (IRSNB). Paratypes: $1 \sigma^{\star}, 1$ ㅇ: same data as holotype (IRSNB); 1 ○': "Alger" (IRSNB).

Melagria biskrensis: Holotype $0^{\top}$ : "Algérie: Ziban, oued Biskra, 17/25-v-1954, G. Fagel / biskrensis n. sp. / Type / Anaulacaspis formosa (Rosenhauer), det. V. Assing 2016" (IRSNB). Paratypes: $2 \sigma^{\top} o^{\top}$ [teneral]: same data as holotype (IRSNB); $10^{\star}:$ "Algérie: Djebel Metlilli, gorges d'El

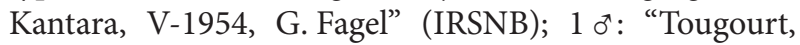
10-20-v-36, Algérien, leg. Vogel” (IRSNB); 1 o : "Algérie: Ziban, oasis de Filiach, 25-v-1954, G. Fagel” (IRSNB); 1 ơ: "Melaha, Trip., 13.4.1936, R. e C. Koch" (IRSNB); $10^{\text {*: }}$ : "Kairouan 10 / Laghruat [?] / R.I.Sc.N.B. 17.479, Falagria naevula Er., Coll. et det. A. Fauvel" (IRSNB); 2 o o : "T. Kairouan, Dr. Normand / R.I.Sc.N.B. 17.479, Falagria naevula Er., Coll. et det. A. Fauvel” (IRSNB); 
1 ơ: $^{\star}$ "Kairouan 1 / R.I.Sc.N.B. 17.479, Falagria naevula Er., Coll. et det. A. Fauvel" (IRSNB).

Melagria cirrosa andalusiaca: Holotype ơ: "Andalousie: S. Pedro Alcantara (Marbella), V.1963, G. Fagel / G. Fagel det., cirrosa andalusiaca nov. / Type / Anaulacaspis cirrosa (Fauvel), det. V. Assing 2016" (IRSNB). Paratypes: $10^{\star}, 1$ ㅇ: same data as holotype; 1 : "Andalousie : Ronda, rio Grande, 8/20-V-1956, G. Fagel / G. Fagel det., cirrosa andalusiaca nov. / Paratype" (IRSNB); $10^{7}, 1$ ㅇ: "Andalousie: Lanjaron (prov. Granada), 26.IV.-18.V.1961, G. Fagel, 85 / G. Fagel det., cirrosa andalusiaca nov. / Paratype" (IRSNB); 1 ㅇ: "Andalousie : Ronda, el Tajo, 16-V-1956, G. Fagel / G. Fagel det., cirrosa andalusiaca nov. / Paratype" (IRSNB).

Comment: The original description of Falagria formosa is based on an unspecified number of syntypes "Bei P. S. Maria und Malaga ... häufig gefunden" (RosenHAUER 1856). FAGEL (1969) designated a female from the Fauvel collection, which he regarded as a syntype, as the lectotype. According to Horn et al. (1990), the remaining type specimens should be in the MNHNP.

The original description of Falagria cirrosa was described from an unspecified number of syntypes from "Algérie et Tunisie" (FAuvel 1902). A lectotype was designated by FAgel (1969). FAUvel (1902) already suspected that A. cirrosa represented "une race bien distincte" of A.formosa (which he erroneously referred to as A. naevula).

The original description of Melagria formosa algirica is based on a male holotype from "Algérie : Philippeville", a paratype from the same locality, and four paratypes from four additional localities in Tunisia and Algeria (FAgEL 1969).

Melagria formosa macra was described from a male holotype and two paratypes from "Algérie : Algérois, Kaddous" and five paratypes from three additional localities in Algeria (FAgEL 1969).

The original description of Melagria formosa elkantarensis is based on a male holotype and "Nombreux exemplaires" from Djebel Metlilli, gorges d'El Kantara”, as well as one paratype from “Aurès, M'Chounech" (FAgEL 1969).

Melagria biskrensis was described based on a male holotype from "Algérie : Ziban, oued Biskra" and additional paratypes from various localities in Algeria, Tunisia, and Libya (FAgel 1969).

The original description of Melagria cirrosa andalusiaca is based on a male holotype and seven paratypes from "Andalousie: Marbella, San Pedro Alcantara, rives du rio Guadalania" and eight paratypes from four additional localities in Andalucía (FAgEL 1969).

An examination of the type material of the above names, as well as of the additional material listed below revealed an enormous variation of external characters such as size, coloration, and the length and width of the elytra. However, the male sexual characters are identical. Moreover, different morphs do not correspond to any plausible distribution patterns and were often found in the same region. Part of the type specimens of different species or subspecies were even collected in the same localities. In consequence, the characters emphasized by FAUVEL (1902) and FAgEL (1969) as distinguishing A. cirrosa, A. biskrensis, and the subspecies of $A$. formosa and $A$. cirrosa from the nominal subspecies of $A$. formosa are interpreted as intraspecific variation of a highly variable species. There is no evidence that this species is represented by geographically distinct subspecies, even though the smaller and more slender morph with smaller elytra is the predominant form in southern Spain and rather rare in North Africa. This multiple synonymy may be explained not only with the pronounced intraspecific variation of $A$. formosa, but also with the observations that some of the type specimens are teneral and that all the specimens originally labelled as A.formosa in the IRSNB are females, whereas the type series of A. biskrensis is exclusively composed of males.

Although part of the type material of Anaulacaspis biskrensis was collected in Libya, neither A. biskrensis nor any of the other names now in the synonymy of A. formosa is listed for Libya in the Palaearctic Catalogue (SChÜLKe \& SMETANa 2015).

Additional material examined: Spain: Murcia: $2 o^{-1} o^{-}$, 1 ㅇ, Murcia env. [“Sp 202”], leg. Franz (NHMW, cAss). Andalucía: $3 \sigma^{\star} o^{\star}, 3$ 우 으, Cádiz, Sierra de Grazalema, El Bosque, rio Malaceite, 26.VI.2003, leg. Santamaria (cAss); 1 ㅇ, Cádiz, $20 \mathrm{~km} \mathrm{~N}$ Tarifa, $36^{\circ} 09^{\prime} \mathrm{N}, 5^{\circ} 38^{\prime} \mathrm{W}$, $100 \mathrm{~m}$, loamy shore of reservoir, flood debris, sifted, 29.XII.2009, leg. Assing (cAss); $10^{x}, 1$ ㅇ․ Malaga, Jubrique, $500 \mathrm{~m}$, stream bank, debris, 26.III.1994, leg. Wunderle (cWun); $90^{\star}$ o $^{*}, 11$ 우 우, Ronda, $500 \mathrm{~m}$, flood debris, 6.III.1990, leg. Matern (cWun, cAss); 1 ơ, 2 ㅇ 우, Sierra de Cazorla [“Sp 687”], leg. Franz (NMW); 2 우 우, Cádiz, $9 \mathrm{~km}$ SE Alcala de los Gazules, bank of Rio Alberite, 7.V.1991, leg. Hieke (MNB); 1 ㅇ, Estepona env. [“Sp 645”], leg. Franz (NHMW); 1 ○, 3 우 우, Estepona, trail to Ronda, 1000 m, X.1986, leg. Benick (MNB); 4 우 우, Estepona, 20.X.1968, leg. Benick (MHNG); 5 우 우, Estepona, X.1968, leg. Benick (MHNG); 1 ơ 1 \% , Estepona, X.1968, leg. Benick (MNB); 1 ㅇ, Malaga, leg. Weise (NHMW); 4 ơ o o $^{\star} 4$ ㅇ 우, Malaga, 20.XI.1980, leg. Simon (NHMW, cAss); 1 , Algeciras (MHNG); 1 , bank of Galadjaro river, 28.-31.V.1968, leg. Benick (MHNG); 1 o, locality not specified (MNB).

Morocco: $4 \diamond^{\top} \sigma^{-}, 4$ 우, Haut Atlas, NE Tizi-nTest, $30^{\circ} 54^{\prime} \mathrm{N}, 8^{\circ} 19^{\prime} \mathrm{W}, 1710 \mathrm{~m}$, stream bank, sifted, 29.XII.2002, leg. Assing (cAss); 2 o $^{\top} o^{\star}, 4$ ㅇ 우, same data, but leg. Wunderle (cWun); $1 \sigma^{\star}, 1$ 우, Haut Atlas, S Tizin-Test, Tachguette, $30^{\circ} 51^{\prime} \mathrm{N}, 8^{\circ} 23^{\prime} \mathrm{W}, 1600 \mathrm{~m}, 30 . \mathrm{IV} .2009$, leg. Behne (cAss); $2 \sigma^{\star} o^{\star}, 2$ 우, Haut Atlas, Tinmal, XI.1985, leg. Rougemont (cAss); 1 ㅇ, Ar Rif, road Chefchaouen-Ksal el Kebr, $35^{\circ} 05^{\prime} \mathrm{N}, 5^{\circ} 25^{\prime} \mathrm{W}, 680 \mathrm{~m}, 3 .-7$. VI.2007, leg. Hlaváč (cAss); 1 ๙ , 1 ㅇ, Ar Rif, Oued Laou

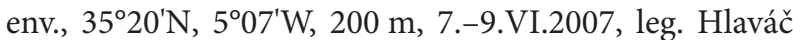

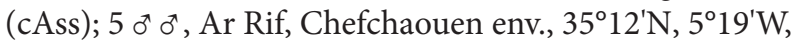


270 m, 3.VI.2007, leg. Hlaváč (cAss); 1 ơ $^{\star} 2$ ㅇ ㅇ, Forêt Mamora, stream on road to Tiflet, $34^{\circ} 04^{\prime} \mathrm{N}, 6^{\circ} 06^{\prime} \mathrm{W}$, 160 m, 26.III.2008, leg. Andújar et al. (cAss); 1 ㅇ, $30 \mathrm{~km}$

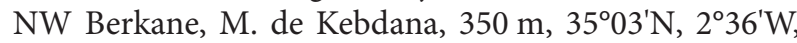
31.XII.2001, leg. Bayer (MNB); 1 ㅇ, Larache env., Jemis del Sahel, 3.IV.1959, leg. Besuchet (IRSNB); 1 ㅇ, Esmir, leg. Walker (IRSNB); $10^{\star}, 1$ \% , Bni Tadjite, Oued Ait Aissa, $32^{\circ} 18^{\prime} \mathrm{N}, 3^{\circ} 28^{\prime} \mathrm{W}, 17 . \mathrm{I} .2015$, leg. Daoudi (cTro); 1 우, Mansouria, IV.1936, leg. Grosclaude (cTro); $2 \sigma^{\top} \sigma^{\star}$, Ouirgane, UV light, 19.V.2015, leg. Miessen (cTro); 5 exs., Marrakesh, leg. Quedenfeldft (MNB); 1 ㅇ, Morbeya river, leg. Quedenfeldt (MNB); 3 우 오, Larache env., Jenis del Sahel, 3.IV.1959, leg. Besuchet (MHNG); $2 \sigma^{\star} \sigma^{*}, 2$ ㅇ 우, "Fl. Issil", leg. Quedenfeldt (NHMW, cAss); $10^{\star}$, locality not specified, leg. Simon (NHMW).

Algeria: $1 \sigma^{\top}$, Biskra, Hammam Salahine, 24.-25.V.1971, leg. Hoffer \& Horák (cAss); $1 \sigma^{\star}$, Ain Zatout, Djebel Aures, 26.V.1971 (cAss); 1 ㅇ, "Lalla Marnia" (IRSNB); 1 ㅇ, Philippeville, Bou Saada (IRSNB); $10^{*}, 2$ ㅇ ㅇ, Bejaia ["Bougie"; $36^{\circ} 45 \mathrm{~N}, 05^{\circ} 05 \mathrm{E}$ ], leg. Théry (NHMW); 1 운, Hammam Righa $\left[36^{\circ} 22^{\prime} \mathrm{N}, 2^{\circ} 23^{\prime} \mathrm{E}\right]$ (MNB); $10^{\star}$, locality not specified, leg. Fauvel (NHMW); 1 ㅇ, Mustapha, leg. Peyerimhoff (NHMW); 1 ㅇ, Annaba (MHNG); $30^{*} o^{*}$, 1 \% , Blidah (MHNG).

Tunisia: $1+$, Jendouba, Ben M’Tir, 8 km S Aïn Draham, $36^{\circ} 43^{\prime} \mathrm{N}, 8^{\circ} 42^{\prime} \mathrm{E}, 610 \mathrm{~m}, 2 . I I I .2012$, leg. Hetzel (cFel);

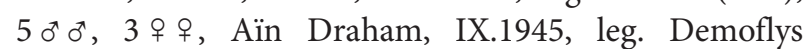
(MHNG, cTro); 1 ㅇ, Sousse, $18 \mathrm{~km} \mathrm{SW} \mathrm{Hammamet,}$ Bouficha, 1.XII.1994, leg. Wrase (cAss); 1 ㅇ, Teboursouk, VII. (IRSNB); 1 o, Jerid, 4 km NE Tozeur, 30-40 m, light trap, 21.X.2009, leg. Lehmann (cAss); 1 ㅇ, Nabeul, coast, 0-30 m, 4.-16.IV.1999, leg. Winkelmann (cAss); $2 o^{\star} o^{\star}, 3$ 우 우, Le Kef (MHNG, NHMW); 1 우, Le Kef, leg. Normand (cTro); $10^{\star}, 4$ 우 인 Le Kef, X.1941, leg. Demoflys (cTro); $3 \sigma^{\star} \sigma^{\star}, 1$ ㅇ, Le Kef, IX.1935, leg. Normand (cTro); $60^{\star} o^{*}, 4$ ㅇ ㅇ, Rades, XII.1936, leg. Grosclaude (cTro, cAss); 3 o o o , 2 우 우, same data, but X.1943 (cTro); 1 ơ $^{*} 2$ 우 우, Rades, X.1941, leg. Demoflys (cTro); 1 우, Gabès, I.1946, leg. Demoflys (cTro); 1 \%, Kairouan, III.1925, leg. Rambousek (MHNG).

Presumably mislabelled: 1 우, “Pyr.-Or." (NHMW); 1 우 "Herault, Palavas" (MHNG).

Redescription: Habitus as in Fig. 211. Body length 2.4-3.3 mm; length of forebody $1.2-1.5 \mathrm{~mm}$. Coloration: head reddish-brown to blackish-brown; pronotum reddish to reddish-brown; elytra reddish-yellow with a more or less distinct and more or less extensive mediolateral infuscate spot; abdomen bicoloured: segments III and IV reddish, segment $\mathrm{V}$ usually at least partly darkbrown to blackish; segments VI-VIII blackish-brown to black; legs yellowish; antennae brown to dark-brown with the basal 3-4 antennomeres yellowish to reddish.

Head relatively weakly transverse, approximately 1.1 times as broad as long, with weakly pronounced sexual dimorphism. Eyes slightly longer than postocular region in dorsal view. Antennae slender, 0.9-1.1 mm long; antennomeres IV-V usually at least weakly oblong,
VI approximately as long as broad, VII-X increasingly transverse, $\mathrm{X}$ approximately 1.5 times as broad as long. Pronotum approximately as broad as long or weakly transverse, and approximately as broad as head; sexual dimorphism usually weakly pronounced; punctation rather dense.

Elytra of hightly variable width and length, 0.8-1.0 times as long as pronotum; punctation very fine and moderately dense. Hind wings present, possibly poly- or dimorphic. Abdomen as broad as, or slightly narrower than, elytra; punctation fine, but distinct, rather dense, and more or less regularly distributed; posterior margin of tergite VIII (Figs 196-197) weakly convex, with a row of distinctly modified short and stout setae.

$\sigma^{*}$ : punctation of head and pronotum on average more distinct than in female; pronotum sometimes more or less depressed or shallowly impressed and/or with short fine median sulcus in postero-median portion; sternite VIII (Fig. 198) with convex posterior margin; median lobe of aedeagus (Figs 199-208) usually 0.36-0.40 mm long (exceptionally larger; see notes on intraspecific variation below), with weakly pronounced crista apicalis; ventral process apically weakly incised in ventral view.

$:$ posterior margin of sternite VIII (Fig. 209) concave in the middle; spermatheca (Fig. 210) minute and commashaped, not distinctive.

Intraspecific variation: Anaulacaspis formosa is subject to enormous intraspecific variation both within and between populations, not only of size, coloration, the length and width of the elytra, as mentioned above, but also of the male modifications of the head and pronotum, and even the size of the aedeagus. In two males from Tunisia and Morocco, the median lobe is distinctly larger (length $0.47 \mathrm{~mm}$; see Figs 199-202) and the pronotum of the male from Tunisia has a postero-median impression. However, the male from Morocco was collected together with other males with aedeagi of normal size and no additional evidence was found suggesting that these specimens represent a distinct species. In any case, the extent of intraspecific variations indicates a patchy distribution and low gene flow between populations.

Comparative notes: As can be inferred from the similarly derived chaetotaxy of the abdominal tergite VIII (posterior margin with strongly modified marginal setae) and from the similar morphology of the aedeagus, A. formosa is evidently the adelphotaxon of the allopatric A. elegans. For characters distinguishing both species see the comparative notes in the following section. The only geographically close and possibly partly sympatric congener is $A$. desertorum, from which $A$. formosa differs by a less transverse head, a more slender and more convex (cross-section) pronotum with a much less pronounced sexual dimorphism, the coloration particularly of the elytra (medio-lateral infuscate spot less defined and less extensive), the modifications of tergite VIII, and by the completely different morphology of the aedeagus. 
Distribution and natural history: Based on the examined material, A. formosa is widespread and rather common in South Spain and North Africa (Morocco, Algeria, Tunisia, Libya) (Map 3). Since this species has been misinterpreted or confounded with other species in the past, literature records from other regions are doubtful. The same applies to the two specimens categorized above as presumably mislabelled.

The specimens for which ecological data are available were hand-collected or sifted from debris on the banks of rivers and streams, or they were caught with light traps. Teneral specimens were found in May. The altitudes range from 100 to $1710 \mathrm{~m}$.

\section{Anaulacaspis elegans (BAUDI DI SELVE, 1857) (Figs 212-220, Map 3)}

Falagria elegans BAUdi di SELVE, 1857: 97.

Comment: The original description is based on an unspecified number of syntypes collected "in insula Cypro" (BAudi 1857). According to Fagel (1969), who designated a lectotype, there are two type specimens, a male and a female, in the Baudi collection. A loan request addressed to the current curator at the museum in Torino, where the Baudi collection is deposited, remained without reply. Although no material of A. elegans from Cyprus was available, there is little doubt that the present interpretation of A. elegans is correct, since this is the only species in the region that matches the details indicated in the original description and FAGEL (1969), who examined the types, reports numerous records from the Middle East.

Material examined: Lebanon: $4 o^{\star} o^{\star}, 2$ $~ ㅇ$, Beyrouth, 1878, leg. Appl (NHMW, cAss); 1 ㅇ, locality not specified, leg. Hofmann (NHMW).

Israel: $9 \sigma^{\top} \sigma^{\top}, 4$ 우 우, Wadi Qelt, St. George Monastery, $31^{\circ} 50^{\prime} \mathrm{N}, 35^{\circ} 24^{\prime} \mathrm{E},-110 \mathrm{~m}, 15 . I I I .2011$, leg. Meybohm (cAss); 4 우 우, West Bank, Wadi Qelt, St. George Monastery, $31^{\circ} 51^{\prime} \mathrm{N}, 35^{\circ} 25^{\prime} \mathrm{E},-70 \mathrm{~m}$, stream bank, 15.III.2011, leg. Hetzel (cFel); 1 오, En Prat Nature Reserve, $31^{\circ} 50^{\prime} \mathrm{N}$, $35^{\circ} 19^{\prime} \mathrm{E}, 230 \mathrm{~m}, 13 . \mathrm{III} .2011$, leg. Meybohm (cAss); $5{o^{\star} o^{\star}}^{*}, 3$ ㅇ 우, Lake Genezareth, Jordan river, $32^{\circ} 54^{\prime} \mathrm{N}$, 353'ㄹ, -215 m, 19.III.2011, leg. Meybohm (cAss); 1 o $^{\circ}$,

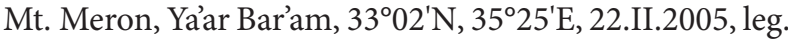
Aßmann (cFel); $10^{\star}, 1$ ㅇ, Dead Sea region, Wadi Boqeq, $31^{\circ} 12^{\prime} \mathrm{N}, 35^{\circ} 21^{\prime} \mathrm{E},-370 \mathrm{~m}, 27 . I I I .2011$, leg. Meybohm (cAss); $1 \sigma^{\star}$, Dead Sea region, Ein Gedi, Wadi Arugot, 20.XI.2009, leg. Drees (cFel); $40^{\star} 0^{\star}, 17$ 우 우, Negev Desert, Wadi En Avdat, 30³6'N, 34²47'E, 850 m, 30.III.2011, leg. Meybohm (cAss); $30^{*} o^{\star}, 1$ \% , Ma'agar, Yeroham, 305 $59^{\prime} \mathrm{N}$, $34^{\circ} 54^{\prime} \mathrm{E}, 450 \mathrm{~m}$, pitfall, 3.VIII.2010, leg. Drees (cAss); $20^{\star} o^{\star}, 3$ 우 우, same data, but 14.III.2011 (cAss); $10^{\star}, 1$ 우, Negev, Yeroham Reservoir, 3059' N, 34 $54^{\prime} \mathrm{E}, 460 \mathrm{~m}$, 28.II.2009, leg. Starke (cFel); $10^{\star}$, Negev, W Ofakim, Ha-Bsor road, $31^{\circ} 17^{\prime} \mathrm{N}, 34^{\circ} 29^{\prime} \mathrm{E}, 2 . X I I .2007$, leg. Aßmann
(cAss); 1 o $^{\prime}$, Ma'agan, $32^{\circ} 43^{\prime} \mathrm{N}, 35^{\circ} 45^{\prime} \mathrm{E},-210 \mathrm{~m}$, tamarisk plain, 26.IV.2010, leg. Drees (cAss); $10^{\star}, 1$ ㅇ, same data, but 30.I.2011 (cAss); 1 우 , same data, but 16.VII.2010 (cFel); 7 우 오, En Avedat, 700 m, spring, 24.IV.2011, leg.

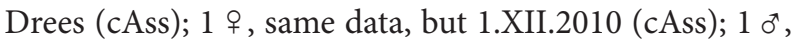
Shoham, $32^{\circ} 00^{\prime} \mathrm{N}, 34^{\circ} 58^{\prime} \mathrm{E}, 140 \mathrm{~m}$, pitfall, 15.XI.2010, leg. Drees (cAss); $10^{\circ}$, Upper Galilee, Zivon, $33^{\circ} 01^{\prime} \mathrm{N}$, $35^{\circ} 25^{\prime} \mathrm{E}, 29 . I V .2006$, leg. Aßmann (cFel); 1 오, 2 우 우, Upper Galilee, north shore of Sea of Galilee, between Kfar Nakhum and Jordan river, $-200 \mathrm{~m}$, tamarisk floodplain forest, 31.III.-1.IV.2008, leg. Wrase (MNB, cAss); $160^{\top} o^{\top}$, 16 우 우 [partly teneral], Upper Galilee, north shore of Sea of Galilee, Kfar Nakhum (Capernaum), 32 $53^{\prime} \mathrm{N}, 35^{\circ} 35^{\prime} \mathrm{E}$, $-200 \mathrm{~m}$, shore with stones and gravel, 22.IV.2006, leg. Wrase (MNB, cAss); $20^{\star} o^{\star}, 1$ ㅇ, Sea of Galilee, Capernaum, Jordan river, 26.III.2008, leg. Aßmann (cFel, cAss); $1 o^{\star}, 1$ ㅇ , Capernaum, $32^{\circ} 53^{\prime} \mathrm{N}, 35^{\circ} 35^{\prime} \mathrm{E}$, 19.IV.2005, leg. Aßmann (cFel); 1 + , same data, but 9.III.2007 (cFel); $10^{\star}$, same data, but 26.III.2008 (cFel); $2 \sigma^{\top} \sigma^{\star}, 5$ 우 우 , Upper Galilee, north shore of Sea of Galilee, Kfar Nakhum, $32^{\circ} 53^{\prime} \mathrm{N}, 35^{\circ} 35^{\prime} \mathrm{E},-200 \mathrm{~m}$, shore with stones and gravel, 12.III.2008, leg. Wrase (MNB, cAss); $20^{\star} 0^{\star}, 4$ 우 우, Upper Galilee, Jordan river near north shore of Sea of Galilee, $-200 \mathrm{~m}$, loamy bank with Cardamine officinalis, 26.III.2008, leg. Wrase (MNB, cAss); $10^{\star}$ [teneral], 1 우 Sea of Galilee, Beit Tsaida Reserve, $32^{\circ} 53^{\prime} \mathrm{N}, 35^{\circ} 38^{\prime} \mathrm{E}$, 0 m, 19.IV.2005, leg. Aßmann (cFel); 1 ๙ ๙ $^{*} 3$ 우 우, Upper Galilee, Batika N.R., Batika river near north shore of Sea of Galilee, -200 m, fine gravel bank, 26.III.2008, leg. Wrase (MNB, cAss); $10^{\star}$, North District, Upper Galilee,

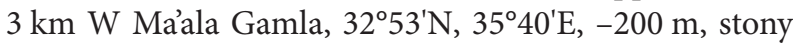
stream bank, 22.IV.2006, leg. Wrase (cAss); $10^{\star}, 5$ 우 우, Central District, Nitsanim, dunes between Ashdod and Ashkelon, 29.III.2008, leg. Wrase (MNB, cAss); 3 우 우, Central District, SW Khadera, brackish pond, 27.III.2008, leg. Wrase (MNB, cAss); 1 o $^{\star} 1$ \%, South District, Negev, W Yerukham, Reem, in irrigated soil, 19.III.2008, leg. Wrase (MNB, cAss).

Jordan: 3 ㅇ ㅇ , Madaba, Wadi Manshala, $31^{\circ} 41^{\prime} \mathrm{N}, 35^{\circ} 35^{\prime} \mathrm{E}$, -370 m, 4.III.2015, leg. Hetzel, Meybohm, Ziegler (cAss, cFel); 2 우 우, Madaba, Wadi Manshala, $31^{\circ} 41^{\prime} \mathrm{N}, 35^{\circ} 35^{\prime} \mathrm{E}$, -280 m, 1.III.2014, leg. Meybohm (cAss); 1 우, Wadi Rum, Camp Site, $29^{\circ} 29^{\prime} \mathrm{N}, 35^{\circ} 23^{\prime} \mathrm{E}, 970$ m, 27.II.2014, leg. Meybohm (cAss); $10^{\star}, 1$ 우, Wadi Rum, Lawrence Spring, $29^{\circ} 34^{\prime} \mathrm{N}, 35^{\circ} 25^{\prime} \mathrm{E}, 1010 \mathrm{~m}, 11 . I I I .2014$, leg. Hetzel (cFel, cAss); $20^{\star} 0^{\star}, 3$ 우 오, Irbid, Wadi Yabis, $32^{\circ} 24^{\prime} \mathrm{N}, 35^{\circ} 35^{\prime} \mathrm{E}$, -210 m, 3.III.2014, leg. Hetzel (cFel, cAss); 1 , Ma’an, $\mathrm{N}$ Wadi Musa, $30^{\circ} 24^{\prime} \mathrm{N}, 35^{\circ} 30^{\prime} \mathrm{E}, 1670 \mathrm{~m}$, 9.III.2014, leg. Hetzel (cFel); 2 o $^{\star}$, S Madaba, Wadi Wala, $500 \mathrm{~m}$, 9.II.1958, leg. Klapperich (MHNG).

Saudi Arabia: 1 ㅇ, Al Bahah, W Elzaraeb, $20^{\circ} 04^{\prime} \mathrm{N}$, $41^{\circ} 23^{\prime} \mathrm{E}, 2090 \mathrm{~m}, 9 . \mathrm{V} .2011$, leg. Sharaf (cAss).

Egypt: 10 , "Sinaï" (NHMW).

Redescription: Body length 2.2-3.2 mm; length of forebody $1.2-1.5 \mathrm{~mm}$. Coloration variable: head reddish to dark-brown; pronotum pale-reddish to reddish-brown; elytra yellowish to yellowish-red with a more or less 
distinct and more or less extensive medio-lateral infuscate spot; abdomen bicoloured: segments III and IV reddish and segments V-VIII dark-brown to blackish; legs yellowish; antennae reddish to dark-brown with the basal 2-3 antennomeres yellowish to reddish.

Head relatively weakly transverse, approximately 1.1 times as broad as long, with weakly pronounced sexual dimorphism; punctation dense, fine, but distinct. Eyes much longer than postocular region in dorsal view. Antennae slender, 0.9-1.1 mm long; antennomeres IV-V usually at least weakly oblong, VI-VII approximately as long as broad, VIII-X increasingly transverse, $\mathrm{X}$ barely 1.5 times as broad as long.

Pronotum weakly transverse and approximately as broad as head; sexual dimorphism pronounced; punctation dense.

Elytra approximately as long as pronotum; punctation very fine and moderately dense. Hind wings fully developed.

Abdomen as broad as, or slightly narrower than, elytra; punctation fine, but distinct, rather dense, and more or less regularly distributed; posterior margin of tergite VIII (Figs 212, 218-219) convex or obtusely pointed in the middle, with a row of distinctly modified short and stout setae.

$\sigma^{*}$ : punctation of head and pronotum on average more distinct than in female; pronotum with pronounced and extensive median impression, this impression deeper posteriorly than anteriorly; posterior margin of sternite VIII (Fig. 213) broadly convex or weakly pointed in the middle; median lobe of aedeagus (Figs 214-217) 0.40-0.46 mm long; ventral process rather long, straight in lateral view, and apically weakly incised in ventral view.

ㅇ: posterior margin of sternite VIII concave in the middle (Fig. 220).

Comparative notes: As can be inferred from the similar external and sexual characters, and particularly from the synapomorphically derived chaetotaxy of tergite VIII (posterior margin with distinctly modified marginal setae), A. elegans is closely allied to the allopatric $A$. formosa, from which it is distinguished above all by the pronounced impression on the male pronotum and the much longer ventral process of the aedeagus. It differs from the sympatric and syntopic A.desertorum by a usually more dark-yellowish posterior margin of the elytra, longer antennae, a less transverse pronotum, less densely punctate elytra and abdomen, modified setae at the posterior margin of tergite VIII, and a median lobe of the aedeagus with a longer and differently shaped ventral process.

Distribution and natural history: Anaulacaspis elegans is distributed in Cyprus and the Middle East (Lebanon; Israel; Jordan; Egypt: Sinai Peninsula; Saudi Arabia) (Map 3). As can be inferred from the label data, the specimens were primarily collected on the banks of streams and rivers, as well as on lake shores at a wide range of altitudes (-370-1670 m). Teneral specimens were found in April.

\section{Anaulacaspis beesoni (CAMERon, 1939) \\ (Figs 221-228, Map 7)}

Falagria (Melagria) beesoni CAMERon, 1939: 260.

Type material examined: Lectotype $\sigma^{*}$, present designation: "Arni Gad. Mussoorie. / Dr. Cameron. 12.VI.21. / F. Beesoni Cam. Type / M. Cameron. Bequest. B.M. 1955-147. / Syntype / Lectotypus o Falagria beesoni Cameron, desig. V. Assing 2016 / Anaulacaspis beesoni (Cameron), det. V. Assing 2016" (BMNH). Paralectotypes: 4 ㅇ 우: same data as lectotype (BMNH).

Comment: The original description is based on an unspecified number of syntypes from "Mussooree district: Arni Gad ; Keyarkuli” (CAMERon 1939).

Additional material examined: India: $1 \sigma^{\star}$, Uttar Pradesh, Kumaon Hills, Nainital, 1900-2200 m, 25.-30.VI.1989, leg. Schuh (cAss).

Redescription: Body length 1.9-2.1 mm; length of forebody $0.9-1.0 \mathrm{~mm}$. Coloration: body black; legs yellowish-brown to blackish-brown with paler tarsi; antennae brown to blackish, usually with the basal antennomeres paler.

Head transverse, approximately 1.15 times as broad as long, with weakly pronounced sexual dimorphism; punctation extremely fine and moderately dense. Eyes longer than postocular region in dorsal view. Antenna short, approximately $0.65 \mathrm{~mm}$ long, and distinctly incrassate apically; antennomeres IV-X distinctly transverse and of gradually increasing width, $\mathrm{X}$ approximately twice as broad as long.

Pronotum strongly transverse, approximately 1.2 times as broad as long and slightly broader than head, broadest at anterior angles, and strongly tapering posteriad; posterior angles nearly obsolete; without appreciable sexual dimorphism; punctation moderately dense, slightly more distinct than that of head.

Elytra approximately as long as pronotum; punctation dense and fine. Hind wings fully developed.

Abdomen slightly narrower than elytra; anterior impressions of tergites III-V deep and without punctation; punctation of remainder tergites III-VI moderately fine and moderately dense; tergite VII with sparser and finer punctation; interstices without appreciable microsculpture and glossy; tergite VIII (Fig. 221) postero-laterally with a cluster of conspicuously modified, apically dilated setae on either side, anterior margin with very shallow median excision, posterior margin convex and with dense and rather long unmodified marginal setae. 
$\sigma^{7}$ : head with shallow and more or less extensive median impression; sternite VIII (Fig. 222) strongly transverse, postero-laterally with a cluster of conspicuously modified, apically dilated setae on either side (Fig. 223), posterior margin broadly and convexly produced in the middle, and with thin marginal setae; median lobe of aedeagus $0.23-0.25 \mathrm{~mm}$ long and shaped as in Figs 224-227.

ㅇ : head not depressed in postero-median portion; sternite VIII (Fig. 228) of similar shape and chaetotaxy as that of male.

Comparative notes: This species is characterized particularly by the strongly transverse pronotum, the presence of clusters of conspicuously modified setae on tergite and sternite VIII, the shallow median excision of the anterior margin of tergite VIII, and the morphology of the median lobe of the aedeagus.

Distribution: The known distribution is confined to Uttarakhand and Uttar Pradesh, North India (Map 7).

\subsection{Species of doubtful generic assignment or of doubtful identity}

"Anaulacaspis" inexpectata (FageL, 1969)

(Figs 229-236, Map 5)

Melagria inexpectata FAgEL, 1969: 41 ff.

Type material examined: Holotype $\sigma^{\star}$ : “Tokmak / R.I.Sc.N.B. 17.479, Coll. et det. A. Fauvel / / G. Fagel det. 1969, Melagria inexpectata n. sp. / Type / Anaulacaspis inexpectata (Fagel), det. V. Assing 2016" (IRSNB). Paratype : same data as holotype (IRSNB).

Comment: The original description is based on a male holotype and a female paratype from "Tokmak" (FAGEL 1969).

Additional material examined: Iran: Azarbayjan-e Gharbi: $20^{\top} o^{*}$ [1 teneral], 1 \%, $20 \mathrm{~km}$ W Salmas, $10 \mathrm{~km}$ W Kuzeh Rash, $38^{\circ} 11^{\prime} \mathrm{N}, 44^{\circ} 31^{\prime} \mathrm{E}, 2100$ m, 31.VIII.2008, leg. Frisch \& Serri (MNB); $10^{\star}$, N Takab, 3 km NE Takhte-Soleyman, $36^{\circ} 38^{\prime} \mathrm{N}, 47^{\circ} 14^{\prime} \mathrm{E}, 2270 \mathrm{~m}$, 8.IX.2008, leg. Frisch \& Serri (cAss).

Redescription: Body length $2.0-2.3 \mathrm{~mm}$; length of forebody $0.90-1.05 \mathrm{~mm}$. Coloration: body black, with the postero-sutural portion of the elytra sometimes slightly paler; legs yellowish-brown to dark-brown; antennae blackish-brown to black. Whole body without microsculpture.

Head weakly transverse, approximately 1.1 times as broad as long, without appreciable sexual dimorphism; punctation fine and moderately dense. Eyes slightly longer than postocular region in dorsal view. Antenna short, approximately $0.7 \mathrm{~mm}$ long; antennomere IV weakly transverse; antennomeres $\mathrm{V}-\mathrm{X}$ of gradually increasing width and increasingly transverse, $\mathrm{X}$ approximately twice as broad as long.

Pronotum weakly transverse, $1.10-1.15$ times as broad as long, and approximately as broad as, or slightly narrower than head, without appreciable sexual dimorphism; punctation dense and fine, denser and more distinct than that of head; midline with fine and shallow median sulcus.

Elytra approximately as long as pronotum; punctation moderately dense and fine, but distinct. Scutellum coarsely and rugosely sculptured. Hind wings fully developed.

Abdomen as broad as, or slightly narrower than elytra; anterior impressions of tergites III-V deep and with a transverse row of very coarse punctures separated by narrow ridges; punctation of remainder of tergal surfaces distinct and moderately dense; tergite VIII (Figs 233-234) without a cluster of long postero-lateral setae, anterior margin without the usual median excision, posterior margin convex and with conspicuous dense and strongly modified, short and stout marginal setae.

$\sigma^{*}$ : head indistinctly depressed in postero-median portion; sternite VIII (Fig. 235) smoothly convex posteriorly; median lobe of aedeagus (Figs 229-232) approximately $0.25 \mathrm{~mm}$ long, ventral process in lateral view straight and without median membranous projection at base, in ventral view narrow and apically convex.

$\uparrow:$ head not depressed in postero-median portion; posterior margin of sternite VIII (Fig. 236) broadly convex; spermatheca not comma-shaped.

Comparative notes: This species is readily distinguished from all its West Palaearctic congeners by several unique character conditions: the coarsely and rugosely sculptured scutellum, the coarse punctation of the anterior impressions of tergites III-V, the conspicuous chaetotaxy of the abdominal tergite VIII, the absence of a median excision of the anterior margin of tergite VIII, the morphology of the median lobe of the aedeagus, and the different shape of the spermatheca. For additional comments see the section on species groups in chapter 3.1 .

Distribution: The type locality "Tokmak" is ambiguous; there are several geographic entities with that name in Ukraine, Russia, and Middle Asia. According to the Palaearctic Catalogue (Schülke \& SMetana 2015), Tokmak is in Kyrgyzstan and thus identical to what is Tokmok today. The additional material was collected in two localities in North Iran (Map 5). 


\section{Anaulacaspis medvedevi (KASHCHEEV, 1982)}

Falagria medvedevi KashCHEEv, 1982: $538 \mathrm{f}$.

Comment: This name was made available in an article containing descriptions of Staphylinidae species of Kyzylkum desert in Kazakhstan (KashCEEV 1982). According to the original description, which is based on several specimens from one locality, which does not specify any characters allowing a reliable interpretation, and whose illustrations of the aedeagus are too schematic to be of any use, $A$. medvedevi resembles $A$. lutzi (i.e., A. gratilla) in habitus and coloration. A loan request addressed to the Zoological Institute in Almaty, where the type material is deposited, remained unanswered. It appears rather unlikely that $A$. medvedevi should represent a species not treated in the present revision. In fact, the sketch of the median lobe of the aedeagus, in particular the shape of the apical membranous protrusion of the internal sac, would suggest that $A$. medvedevi is conspecific with A. gratilla. Until the type material can be accessed, however, the identity of $A$. medvedevi must remain doubtful.

\section{Acknowledgements}

My thanks are due to the colleages indicated in the material section for the loan of material from the collections under their care. Maria Salnitska (St. Petersburg) kindly helped in clarifying the whereabout of the type material of A. medvedevi. Alexey Solodovnikov (Copenhagen) translated the original description of $A$. medvedevi. Marc Tronquet (Molitg-les-Bains) provided the habitus photo of A. formosa. Benedikt Feldmann (Münster) reviewed and proof-read the manuscript.

\section{References}

Assing, V. 1997: A revision of the Eastern Palaearctic species of Myrmecopora SAULCY, 1864 with notes on some species from the Oriental region (Coleoptera: Staphylinidae, Aleocharinae, Falagriini). - Beiträge zur Entomologie, Berlin 47 (2): 337-352.

Assing, V. 2001: Two new species and a new name of Cordalia JacoBs, 1925 from Turkey and China (Insecta: Coleoptera: Staphylinidae: Aleocharinae). Reichenbachia 34 (12): 113-118.

Assing, V. 2009: New species and additional records of Palaearctic Falagriini (Coleoptera: Staphylinidae: Aleocharinae). - Linzer Biologische Beiträge 41 (1): 471-480.

Baudi di Selve, F. 1857: Coleoptera quaedam e Staphylinorum familia nova vel minus cognita cum observationibus. - Berliner Entomologische Zeitschrift 1: 97-115.

Bernhauer, M. 1908: 14. Folge neue Staphyliniden der paläarktischen Fauna, nebst Bemerkungen.
- Verhandlungen der Kaiserlich-Königlichen Zoologisch-Botanischen Gesellschaft in Wien 58: 32-41.

Bernhauer, M. 1910: Zur Staphylinidenfauna des palaearktischen Gebietes. - Societas Entomologica 25: 71-72, 78-79.

Cameron, M. 1925: Descriptions of new species of Oriental Staphylinidae. - Annali del Museo Civico di Storia Naturale Giacomo Doria 52: 34-49.

Cameron, M. 1933: Staphylinidae (Col.) from Mount Kinabalu. - Journal of the Federated Malay States Museums 17: 338-360.

Cameron, M. 1936: Fauna Sumatrensis Bijdrage No. 77, Staphylinidae (Coleoptera). - Tijdschrift voor Entomologie 79: 1-24.

CAMERon, M. 1939: The Fauna of British India, including Ceylon and Burma. Coleoptera. Staphylinidae. Vol. IV. Parts I-II. - London: 691 pp.

Cameron, M. 1944: Descriptions of new Staphylinidae (Coleoptera). - The Proceedings of the Royal Entomological Society of London (B) 13: 121-15, 49-52, 104-108.

Cameron, M. 1950: New species of Staphylinidae (Col.) from the Malay Peninsula. - The Annals and Magazine of Natural History (12) 3: 1-40, 89-131.

Coiffait, H. 1982: Nouveaux staphylinides afghans du Muséum A. Koenig de Bonn. - Bonner Zoologische Beiträge 33 (1): 75-97.

Duvivier, A. 1883: Énumération des staphylinides, décrits depuis la publication du catalogue de M.M. Gemminger et de Harold. - Annales de la Société Entomologique de Belgique 27: 91-215.

Eppelsheim, E. 1880: Falagria laeviuscula n. sp. - In: Reitter, E.: Coleopterologische Ergebnisse einer Reise nach Croatien, Dalmatien und der Herzegowina im Jahre 1879. - Verhandlungen der KaiserlichKöniglichen Zoologisch-Botanischen Gesellschaft in Wien 30: 201-228.

ERICHSON, W. F. 1839: Erster Band. Pp. 1-400. - In: Genera et species Staphylinorum insectorum coleopterorum familiae. - Berlin: F.H. Morin: viii + 954 pp.

FAGEL, G. 1966: Contribution a la connaissance des Staphylinidae (Coleoptera). XCII. - Espèces nouvelles ou méconnues de la méditerranée orientale. - Bulletin et Annales de la Société Royale d'Entomologie de Belgique 102: 21-55.

FAGEL, G. 1969: Contribution à la connaissance des Staphylinidae. XIX. Les Melagria CASEy de la région paléarctique occidentale. - Bulletin Institut Royal des Sciences Naturelles de Belgique 45 (34): 1-43.

Fauvel, A. 1898: Catalogue des Staphylinides de la Barbarie et des Îles Açores, Madères, Salvages et Canaries. Supplément. Description des espèces nouvelles. - Revue d'Entomologie 17: 93-113.

Fauvel, A. 1902: Catalogue des Staphylinides de la Barbarie de la Basse-Égypte et des Iles Açores, Madères, Salvages et Canaries. - Revue d'Entomologie 21: 45-189. 
Fenyes, A. 1912: Falagria Mannerheim and its relatives. - Journal of the New York Entomological Society 20 (1): 20-27.

Gamarra, P. \& Outerelo, R. 2005: Catálogo iberobalear de los Aleocharinae (Coleoptera: Staphylinidae). Boletín Sociedad Entomológica Aragonesa 37: 1-81.

Gravenhorst, J. L. C. 1802: Coleoptera microptera Brunsvicensia. - Brunsuigae, 206 pp.

Gravenhorst, J.L.C. 1806: Monographia Coleopterorum Micropterorum. - Göttingen: 236 pp.

Heer, O. 1839: Fauna Coleopterorum Helvetica. Pars 1. Turici [Zürich]: 145-364.

Hoebeke, E. R. 1985: A revision of the rove beetle tribe Falagriini of America north of Mexico (Coleoptera: Staphylinidae: Aleocharinae). - Journal of the New York Entomological Society 93 (2): 913-1018.

Horion, A. 1967: Faunistik der mitteleuropäischen Käfer. Bd. XI: Staphylinidae, 3. Teil: Habrocerinae bis Aleocharinae (ohne Subtribus Athetae). Überlingen-Bodensee: 419 pp.

Horn, W.; Kahle, I.; Friese, G. \& Gaedike, R. 1990: Collectiones entomologicae. Ein Kompendium über den Verbleib entomologischer Sammlungen der Welt bis 1960. - Akademie der Landwirtschaftswissenschaften der Deutschen Demokratischen Republik, Berlin: 573 pp.

Kashcheev, V. A. 1982: Novye vidy Zhukov-Stafilinov (Coleoptera, Staphylinidae) iz Pustyni Kyzylkum. Entomologicheskoe Obzrenie 61 (3): 537-541.

KocH, C. 1936: Wissenschaftliche Ergebnisse der entomologischen Expeditionen Seiner Durchlaucht des Fürsten Alessandro C. della Torre e Tasso nach Aegypten und auf die Halbinsel Sinai. - Pubblicazzioni del Museo Entomologico "Pietro Rossi" (Duino) 1: 115-232.

LATTIN, G. DE 1967: Grundriss der Zoogeographie. Gustav Fischer Verlag, Stuttgart: 602 pp.

Lohse, G. A. 1974: Tribus 1-13 (Deinopsini-Falagriini). - In: Freude, H.; Harde, K. W. \& Lohse, G. A. (eds.): Die Käfer Mitteleuropas, Bd. 5, Krefeld: 11-71.

LOKAY, E. 1905: Coleoptera myrmecophila bohemica. - Časopis České Společnosti Entomologické 2 (2): 33-50.

Newton, A. F. 2015: New nomenclatural and taxonomic acts, and comments: Staphylinidae; pp.9-15. - In: LöвL, I. \& LöвL, D. (eds.), Catalogue of Palaearctic Coleoptera. Volume 2/1. Hydrophiloidea - Staphylinoidea. Revised and updated edition. Volume 1. - Leiden and Boston: Brill: i-xxv, 1-900.

PACE, R. 1986a: Aleocharinae della Thailandia e della Birmania riportate da G. de Rougemont (Coleoptera, Staphylinidae). - Bolletino del Museo Civico di Storia Naturale di Verona 11 (1984): 427-468.

PACE, R. 1986b: Aleocharinae dell'Himalaya raccolte da G. de Rougemont (Coleoptera Staphylinidae). - Bolletino del Museo Civico di Storia Naturale di Verona 12 (1985): 165-191.
PACE, R. 1987: Staphylinidae dell'Himalaya Nepalese. AleocharinaeraccoltedalProf. Dr. J. Martens (Insecta: Coleoptera). - Courier des Forschungsinstitutes Senckenberg 93: 383-441.

PACE, R. 1998: Aleocharinae della Cina: Parte II (Coleoptera Staphylinidae). - Revue Suisse de Zoologie 105 (2): 395-463.

PACE, R. 1999: Insectes Coléoptères Staphylinidae Aleocharinae. - Faune de Madagascar, Paris 89: $1-261$.

Pace, R. 2004: Aleocharinae di Thailandia, Cambogia, Laos e Malaysia (Coleoptera, Staphylinidae). Belgian Journal of Entomology 6: 243-302.

PACE, R. 2008: Pronomaeini, Homalotini, Bolitocharini, Diestotini e Falagriini di Taiwan (Coleoptera Staphylinidae). - Bollettino des Museo Civico di Storia Naturale di Verona 32: 137-156.

PACE, R. 2010: Aleocharinae della regione orientale al Museo di Genova (Coleoptera, Staphylinidae). Annali del Museo Civico di Storia Naturale "G. Doria" 102: 95-335.

PACE, R. 2011: New and additional records, new genera and new species of Aleocharinae from Venezuela, Ecuador and Peru (Coleoptera Staphylinidae). Bollettino des Museo Civico di Storia Naturale di Verona 35: 43-70.

Reitter, E. 1909: Fauna Germanica. die Käfer des Deutschen Reiches. Nach der analytischen Methode bearbeitet. II. Band. Schriften des Deutschen Lehrervereins für Naturkunde 24. - Stuttgart: K.G. Lutz: $392 \mathrm{pp}$.

Rosenhauer, W. G. 1856: Die Thiere Andalusiens nach dem Resultate einer Reise zusammengestellt, nebst den Beschreibungen von 249 neuen oder bis jetzt noch unbeschriebenen Gattungen und Arten. Erlangen: T. Blaesing: viii +429 pp.

Roubal, J. 1932: Aus der Praxis des Käfersammlers. XIX. Zum Verzeichnis der mitteleuropäischen myrmekophilen Koleopteren. - Koleopterologische Rundschau 18: 120-125.

SAwADA, K. 1966: Coleoptera Staphylinidae of Afghanistan and W. Pakistan. - In: Kitamura, S. \& YosII, R.: Results of the Kyoto University Scientific Expedition to the Karakorum and Hindukush, 1955. Additional Reports 8: 257-262.

Schülke, M. \& Smetana, A. 2015: Staphylinidae, pp. 304-1134. - In: LöвL, I. \& LöвL, D. (eds), Catalogue of Palaearctic Coleoptera. New, updated Edition. Volume 2. Hydrophiloidea - Staphylinoidea. Revised and updated edition. - Leiden: Brill: xxvi + 1702 pp.

Tottenham, C. E. 1957: Contributions à létude de la faune entomologique du Ruanda-Urundi (Mission P. Basilewsky 1953). CXX. Coleoptera Staphylinidae: Tachyporinae, Pygosteninae (cont.) and Aleocharinae (part). - Annales du Musée Royal du Congo Belge, Tervuren (ser. in $8^{\circ}$ ). Sciences Zoologiques 58: 73-135. 